\title{
Studies on AP-1 Sorting Function and Regulation of Membrane Binding
}

\author{
Dissertation \\ zur Erlangung des Doktorgrades \\ der Mathematisch-Naturwissenschaftlichen Fakultäten \\ der Georg-August-Universität Göttingen
}

vorgelegt von

Karthikeyan Radhakrishnan

aus Krishnagiri (Indien)

Göttingen 2006 
D7

Referent: $\quad$ Prof. Dr. Dr. h.c. Kurt von Figura

Koreferent: Prof. Dr. Hans-Joachim Fritz

Tag der mündlichen Prüfung: 16 January 2007 


\section{To my Parents...}




\section{Contents}

Abbreviations $\quad$ v

1 Introduction 1

1.1 Intracellular vesicle transport pathways . . . . . . . . . . . 2

1.2 Components of Clathrin-coated vesicle transport machinery . . . . 4

1.2.1 Clathrin . . . . . . . . . . . . . . . . 4 4

1.2 .2 Adaptor proteins $\ldots \ldots \ldots \ldots \ldots \ldots$

1.2.2.1 Heterotetrameric Adaptor Protein complexes . . . . 5

1.2.2.2 Monomeric Adaptor Proteins . . . . . . . . . . . 9

1.2 .3 Accessory Proteins $\ldots \ldots \ldots \ldots$. . . . . . . . . . . 12

1.2.3.1 Interaction of AP-1 with accessory Proteins . . . . 13

1.3 Life-cycle of Clathrin Coated Vesicles(CCVs) . . . . . . . . . . . . . 14

1.4 Sorting signals $\ldots \ldots \ldots \ldots \ldots \ldots \ldots \ldots$

1.4 .1 Tyrosine-based motifs $\ldots \ldots \ldots \ldots$. . . . . . . . . 17

1.4 .2 Leu-based motifs . . . . . . . . . . . . . . . . . . 18

1.5 Protein sorting at the TGN and endosomes . . . . . . . . . . . . 19

1.5.1 AP-1 Versus GGA adaptors at the TGN sorting station . . . . . 19

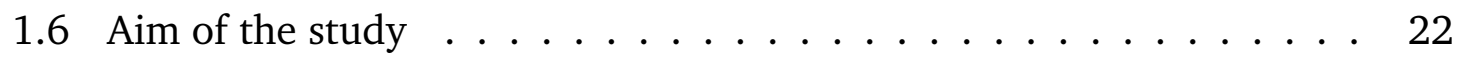

2 Materials and Methods 23

2.1 Materials . . . . . . . . . . . . . . . . . 23

2.1 .1 Laboratory equipment $\ldots \ldots \ldots \ldots$

2.1 .2 Chemicals, plasticware and membranes . . . . . . . . . 24

2.1 .3 Kits, spin columns and reagents . . . . . . . . . . . . . . 24

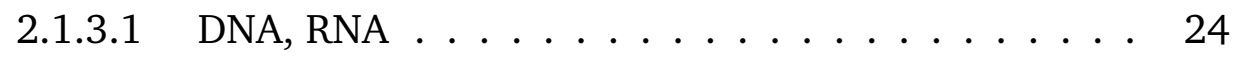

2.1 .3 .2 Protein . . . . . . . . . . . . . . . . . 25

2.1 .4 Strains and Cell lines . . . . . . . . . . . . . . . . . 25

$2.1 .4 .1 \quad$ Bacterial strains $\ldots \ldots \ldots \ldots$. . . . . . . . . 25

2.1 .4 .2 Yeast strains $\ldots \ldots \ldots \ldots \ldots$ 
2.1 .4 .3 Mouse cell lines . . . . . . . . . . . . . 26

2.1 .4 .4 Mouse Strains . . . . . . . . . . . . . 26

2.1 .5 Vectors . . . . . . . . . . . . . . . . 26

2.1.5.1 Mammalian System: . . . . . . . . . . . . . . . . . 26

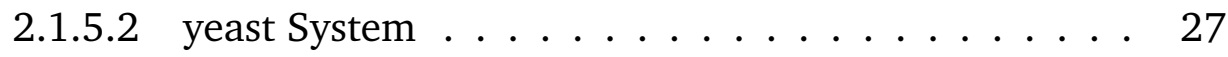

2.1 .5 .3 Bacterial System . . . . . . . . . . . . . . 27

$2.1 .6 \quad$ Antibiotics and drugs . . . . . . . . . . . . . . . . 27

2.1 .7 Enzymes and Standards . . . . . . . . . . . . . . 27

2.1 .8 Primary antibodies . . . . . . . . . . . . . . . . 28

2.1 .9 Secondary antibodies . . . . . . . . . . . . . . . . 28

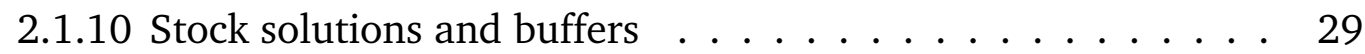

2.2 Methods . . . . . . . . . . . . . . . . . . . 29

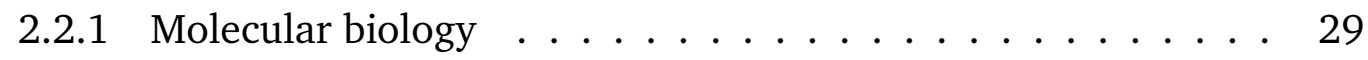

2.2 .1 .1 Cultivation of E.coli . . . . . . . . . . . . . 29

2.2.1.2 Preparation of electrocompetent Bacterial cells . . . 30

2.2.1.3 Transformation of plasmid DNA by Electroporation . 30

2.2.1.4 Preparation of Glycerol stocks for bacterial strains . 31

2.2.1.5 Plasmid DNA preparation and cloning protocols . . 31

2.2.1.6 Determining the concentration of DNA . . . . . . 31

2.2.1.7 $\quad$ Restriction endonuclease digestion of DNA . . . . . 31

2.2 .1 .8 Agarose gel electrophoresis of DNA $\ldots . . . . . .32$

2.2.1.9 Isolation of RNA from mouse cells or mouse tissue . 33

2.2.1.10 Polymerase chain reaction (PCR) . . . . . . . . 33

2.2 .1 .11 Ligation . . . . . . . . . . . . . . 34

2.2 .1 .12 Sequencing PCR . . . . . . . . . . . . . . . . . . . . . . . 34

2.2 .2 Yeast Genetics . . . . . . . . . . . . . . . . . . . . . . 35

2.2.2.1 Lithium acetate mediated yeast transformation . . . 35

2.2 .2 .2 Yeast cell plasmid rescue ................ 36

2.2 .2 .3 Testing interaction by growth . . . . . . . . . . . . 37

2.2 .2 .4 Filter lift assay - lacZ reporter gene . . . . . . . . 37

$2.2 .2 .5 \quad \mathrm{X}$ - $\alpha$-Gal assay . . . . . . . . . . . . 38

$2.2 .3 \quad$ Cell culture and transfections . . . . . . . . . . . . . . 39

2.2 .3 .1 Basics . . . . . . . . . . . . . . . . . 39

2.2 .3 .2 Passaging by trypsinisation . . . . . . . . . . . 39

$2.2 .3 .3 \quad$ Freezing cells for stock maintenance . . . . . . . . . . 39

2.2 .3 .4 Thawing cells. . . . . . . . . . . . 39

2.2 .3 .5 Transient transfection . . . . . . . . . . . . 40

2.2 .4 Biochemical Methods . . . . . . . . . . . . . . . 40 
2.2.4.1 Protein estimation by BIORAD reagent . . . . . . . . 40

$2.2 .4 .2 \quad$ Solubilization of proteins . . . . . . . . . . 40

2.2.4.3 SDS-Polyacrylamide Gel Electrophoresis . . . . . . . 40

2.2 .4 .4 Stacking gel(1X) . . . . . . . . . . . . 41

2.2 .4 .5 Separating gel (1X) . . . . . . . . . . . . 41

2.2.4.6 Staining with Roti-Blue Colloidal Coomassie . . . . 42

2.2.4.7 Western blot (semi-dry) . . . . . . . . . . . 42

2.2 .4 .8 Stripping of nitrocellulose membranes . . . . . . . . 43

2.2.4.9 Preparation of cytosol fraction for pull-down assay . 44

2.2.4.10 purification of GST-fusion proteins . . . . . . . 44

2.2.4.11 Indirect immunofluorescence . . . . . . . . . . 45

2.2.4.12 Trypsin sensitivity assay . . . . . . . . . . 46

2.2.4.13 Fluorescence recovery after photobleaching (FRAP) 47

2.2.4.14 Immunoelectron microscopy . . . . . . . . 47

2.2.4.15 Preparation of whole cell lysate from isolated adipocytes .................. 48

\begin{tabular}{ll|r}
3 & Results & 51
\end{tabular}

3.1 Role of AP-1 in intracellular sorting of sortilin . . . . . . . . . . . . 51

3.1.1 Analysis of the intracellular distribution of sortilin in AP-1 deficient cells .................. 51

3.1 .2 Analysis of interaction between sortilin and AP-1 . . . . . . 56

3.1.2.1 Y2H analysis of sortilin-tail interaction with $\mu 1 \mathrm{~A} . .56$

3.1.2.2 In vitro analysis of sortilin and AP-1 interaction . . . 58

3.1.2.3 Analysis of Interaction between sortilin and $\gamma 1-\sigma 1$ hemi-complex .............. 60

3.1.2.4 Yeast-3-Hybrid analysis . . . . . . . . . . . 60

3.1.2.5 In vitro interaction of sortilin with AP-1 from $\sigma 1 \mathrm{~B}-/-$ cells . . . . . . . . . . . . . 62

3.1.2.6 Analysis of steady-state distribution of Sortilin in o1B-/-cells ................ 63

3.1.3 Analysis of intracellular distribution of Sortilin sorting-motif mutants . . . . . . . . . . . . . . . . 65

3.1.4 Analysis of sortilin in adipocytes isolated from $\sigma 1 \mathrm{~B}$ 'knock-out' mouse ...................... 68

3.2 Specificity of $\gamma 1 / \sigma 1$ adaptin interactions . . . . . . . . . . . 70

3.3 A novel role for the N-terminal domain of $\mu 1 \mathrm{~A}$ in regulation of AP-1

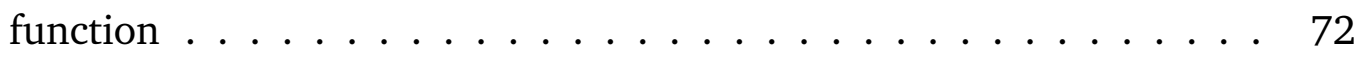


3.3.1 Insights from previous studies on $\mu 2 / \mu 1$-chimeric AP-1 complex 72

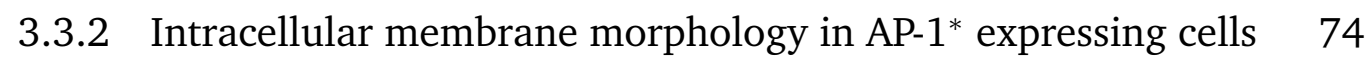

3.3.3 Analysis of AP-1* membrane recycling at the TGN . . . . . . 76

3.3.4 Analysis of known determinants that regulate AP-1/AP-1* membrane binding . . . . . . . . . . . . 79

3.3.4.1 Influence of phosphorylation kinetics on AP-1* membrane binding ............. 80

3.3.4.2 $\mathrm{Y} 2 \mathrm{H}$ analysis of interaction between $\mathrm{N}$-terminal seventy amino acids of $\mu 1 \mathrm{~A}$ and candidate proteins . . 81

3.3.5 Identification of proteins binding to the N-terminal seventy amino acids of $\mu 1 \mathrm{~A} \ldots \ldots \ldots . \ldots \ldots$

3.3.5.1 Analysis of the steady state distribution of AP-1/AP$1^{*}$ in MuL-HA expressing cells . . . . . . . 86

\begin{tabular}{llr}
4 & Discussion & 89 \\
\hline
\end{tabular}

$4.1 \quad$ AP-1 in intracellular sorting of Sortilin . . . . . . . . . . . . . . 89

$4.1 .1 \quad$ Perspective . . . . . . . . . . . . . . . 95

4.2 A role for the N-terminal domain of $\mu 1 \mathrm{~A}$ in regulation of AP-1 function 96

$4.2 .1 \quad$ Influence of the N-terminal domain of $\mu 1 \mathrm{~A}$ on AP-1 mediated

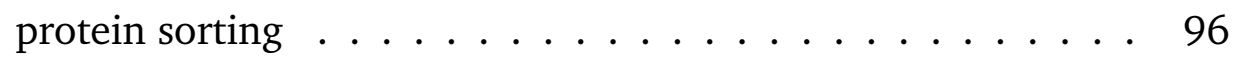

$4.2 .2 \quad$ Role in AP-1 membrane binding dynamics . . . . . . . . . . 97

4.2 .3 A novel protein as a potential regulator of AP-1 membrane

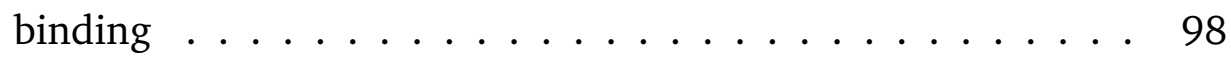

\begin{tabular}{ll}
\hline Summary & 101
\end{tabular}

\begin{tabular}{ll}
\hline References & 103
\end{tabular}

\begin{tabular}{ll}
\hline Acknowledgements & 115
\end{tabular} 


\section{Abbreviations}

$\begin{array}{ll}\text { ADP } & \text { Adenosine diphosphate } \\ \text { AP-1 } & \text { Adaptor protein-complex-1 } \\ \text { APS } & \text { Ammonium peroxide sulfate } \\ \text { ARF } & \text { ADP-Ribosylation Factor } \\ \text { ATP } & \text { Adenosine-5'-triphosphate } \\ \text { BFA } & \text { Brefeldin A } \\ \text { BLAST } & \text { Basic Local Alignment Search Tool } \\ \text { bp } & \text { Base pairs } \\ \text { CCV } & \text { Clathrin-Coated Vesicle } \\ \text { COP } & \text { Coat Protein } \\ { }^{\circ} \text { C } & \text { Grade Celsius } \\ \text { cDNA } & \text { Complementary DNA } \\ \text { Da } & \text { Dalton } \\ \text { ddH2O } & \text { double distilled }{ }_{2} \text { O } \\ \text { DMSO } & \text { Dimethylsulfoxide } \\ \text { DNA } & \text { Deoxyribonucleic acid } \\ \text { DTT } & \text { 1,4-Dithiothreitol } \\ \text { dNTPs } & \text { 2'-Deoxyribonucleoside-5'-phosphate } \\ \text { E.coli } & \text { Escherichia coli } \\ \text { EDTA } & \text { Ethylendiamine-tetra-acetate } \\ \text { ER } & \text { Endoplasmic Reticulum } \\ \text { EST } & \text { Expressed sequence tag } \\ \text { et al } & \text { et alteri (and others) } \\ \text { GGA } & \text { Golgi-localized, } \gamma \text {-ear containing, ARF-binding } \\ \text { GTP } & \text { Guanosine triphosphate } \\ \text { IPTG } & \text { isopropyl- } \beta \text {-D-galactopyranoside } \\ \text { kDa } & \text { kilodalton } \\ 1 & \text { liter } \\ \text { LAMP-1 } & \text { Lysosome Associated Membrane Protein-1 } \\ \end{array}$




\begin{tabular}{|c|c|}
\hline $\begin{array}{l}\text { LB-medium } \\
\mathrm{m}\end{array}$ & $\begin{array}{l}\text { Luria-Broth medium } \\
\text { meter }\end{array}$ \\
\hline $\mathrm{MEF}$ & Mouse Embryonic Fibroblast \\
\hline $\mathrm{mM}$ & milliMolar \\
\hline M & molar \\
\hline$\mu$ & micro, $-\left(\mathrm{x} 10^{-6}\right)$ \\
\hline $\mathrm{mA}$ & milliAmpere \\
\hline $\min$ & minute \\
\hline $\mathrm{MgCl}_{2}$ & Magnesium chloride \\
\hline MPR & mannose-6-phosphate receptor \\
\hline mRNA & messenger RNA \\
\hline MVB & Multi Vesicular Body \\
\hline $\mathrm{n}$ & nano, $-\left(\mathrm{x} 10^{-9}\right)$ \\
\hline OD & Optical density \\
\hline PAGE & Polyacrylamide gel electrophoresis \\
\hline PBS & Phosphate-buffered saline \\
\hline PCR & Polymerase chain reaction \\
\hline PI4P & Phosphotidylinositol 4-phosphate \\
\hline rpm & Rotation per minute \\
\hline RT & Room temperature \\
\hline s & second \\
\hline SDS & Sodium Dodecyl Sulfate \\
\hline $\mathrm{t}$ & time \\
\hline Taq & Thermophillus aquaticus \\
\hline TAE & Tris-acetate-EDTA-buffer \\
\hline TBS & Tris-buffered solution \\
\hline TCA & Trichloro-acetic acid \\
\hline TGN & Trans-Golgi Network \\
\hline $\mathrm{TE}$ & Tris-EDTA \\
\hline TEMED & N,N,N,N-tetramethylethylenediamine \\
\hline Tris & Tris-(hydroxymethyl)-aminomethane \\
\hline UV & Ultraviolet \\
\hline $\mathrm{V}$ & Volt \\
\hline $\mathrm{v} / \mathrm{v}$ & Volume per volume \\
\hline wt & Wild type \\
\hline $\mathrm{w} / \mathrm{v}$ & Weight per volume \\
\hline $\mathrm{Y} 2 \mathrm{H}$ & yeast-two-hybrid \\
\hline $\mathrm{Y3H}$ & yeast-three-hybrid \\
\hline
\end{tabular}




\section{Chapter 1}

\section{Introduction}

A typical mammalian cell contains up to 10,000 different kinds of proteins; a yeast cell about 5000. For proper organization and functioning of an eukaryotic cell,each of its different kind of proteins should be localized to correct cellular membrane or luminal compartment. This highly regulated and co-ordinated process of directing each newly made polypeptide to its appropriate destination is termed as protein sorting or protein targeting. Along the biosynthetic or the secretory pathway, newly synthesized proteins destined for secretion pass through an array of membrane enclosed organelles including the Endoplasmic Reticulum (ER), the Golgi complex and/or secretory granules before they can be secreted to the extra-cellular space. Proteins bound to reside at other compartments like the Plasma Membrane (PM), endosomes or lysosomes share the routes of the secretory pathway. On the other hand, the proteins that are endocytosed at the PM reach their intracellular destinations through the endocytic pathway along routes that overlap with the secretory pathway.

All protein transport pathways along the biosynthetic pathway are mediated by membrane-bound vesicles. The vesicular transport hypothesis was established based on the seminal work of George Palade and co-workers (Palade, 1975) according to which, membrane-bound vesicles harboring cargoes shuttle between organelles of the secretory pathway. The cargoes to be transported are selectively incorporated into forming vesicles from a donor compartment. The membraneous vesicles with cargoes are then subsequently targeted to a specific acceptor compartment, where the cargoes are unloaded by fusion of their limiting membranes.

Such highly accurate trafficking of vesicles along the cytoskeletal filaments is orchestrated and regulated by motor proteins and by a cross-talk between a variety of cytosolic and vesicle bound proteins. 


\subsection{Intracellular vesicle transport pathways}

A detailed picture of the molecular mechanisms involved in selective cargo transport between organelles have been provided by extensive genetic and biochemical analysis of the secretory pathway. The transport vesicles involved in protein traffic

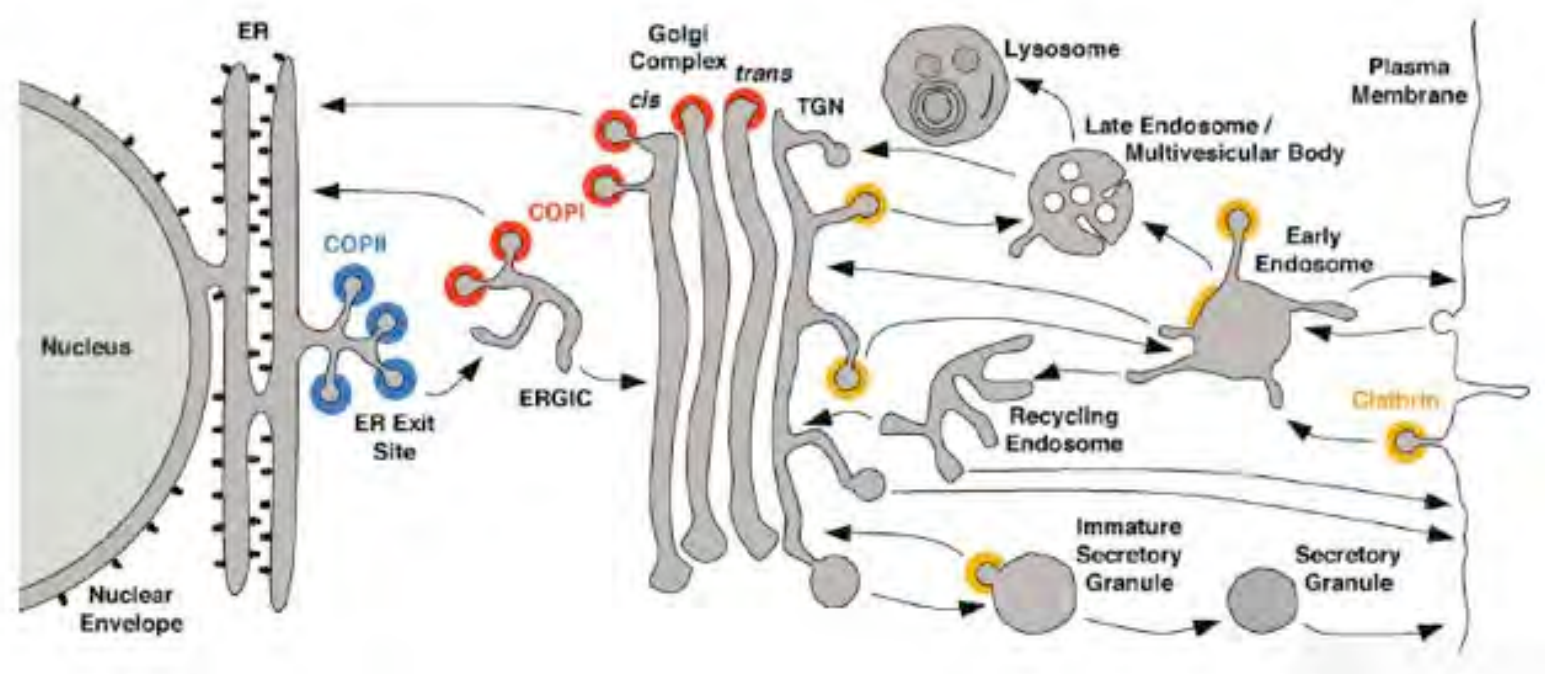

Figure 1.1: Vesicular transport pathways in a mammalian cell. The scheme depicts intracellular protein transport along the secretory, endocytic and lysosomal compartments. Arrows indicate various transport steps. The known or presumed locations of COPI(red), COPII (blue) and clathrin (orange) coats are indicated in colour. Clathrin coats are heterogenous and contain distinct combination of adaptor proteins at different membranes (Bonifacino and Glick, 2004). Additional coats or coat-like components exist but are not depicted in this figure.

in the secretory pathway are equipped with specific protein coats that mediate the budding and selective incorporation of cargo in the forming vesicles (Fig.1.1). Among various types of coats that operate, the most widely studied and known are, the COPI coat and the COPII coatomer complex that function at the early-secretory phase and second, those coats containing clathrin as their main constituent that function exclusively at the post-Golgi sorting events. Apart from the above mentioned pathways, the pathways that are mediated in a, coat-independent mechanism also exist, but are less characterized (Bonifacino and Glick, 2004).

The anterograde and the retrograde transport between ER and Golgi constitute the early-secretory pathway. COPII mediates protein export from ER to Golgi (anterograde) either directly or through a transient intermediate called the ERGolgi Intermediate Compartment (ERGIC) (Barlowe et al., 1994). Meanwhile COPI coatomer mediates transport from the Golgi to ER (retrograde) and also intra-Golgi transport. COPII coats assemble at specialized ER exit sites (also called transitional 
ER sites), which are generally devoid of ribosomes. Coat assembly begins through activation of small G-proteins; Sar1p for COPII and ARF1(ADP-ribosylation factor 1) for COPI and these activated G-proteins recruit the coat proteins. The COPI coatomer complex consists of seven subunits that are organised into one trimeric and two dimeric subcomplexes and the COPII coat has four subunits. Both COPII and COPI coats recognize the sorting signals present in the cytoplasmic tails of membrane cargo proteins.

When a protein to be secreted reaches trans-Golgi network (TGN), further transport between the TGN and the plasma membrane(PM) is maintained by clathrincoated vesicles(CCVs). As shown in Fig. 1.1, TGN acts as a major sorting station and a transport vesicle budding from the TGN can take different routes as dictated by the nature of cargo and the type of clathrin associated adaptor protein (AP) complex. Several different types of adaptor proteins and accessory proteins are found to be associated with clathrin(discussed in detail in the next section). By the constitutive secretory pathway, surface-destined cargo is delivered directly from TGN to the PM by a less understood mechanism and the precise identities of the proteins mediating constitutive secretion at TGN remains to be established. However, recently two proteins called FAPP1 and FAPP2 (four phosphate-adaptor protein 1 and 2) were identified to be components of a phosphotidylinositol-4-phosphate(PtdIns(4)P) and ARF-regulated machinery involved in the generation of constitutive TGN to PM carriers (Godi et al., 2004). In contrast to the constitutive pathway, cargoes in clathrin-coated vesicles can be vectorially delivered to the lysosomes or the PM through the internal endosomal system. The endosomal system acts as a sorting substation; anterograde pathways deliver the cargo to the lysosomes and PM and by retrograde pathway deliver back to the TGN. In cells that undergo signal-dependent regulated secretion, proteins are first incorporated into the secretory granules called immature secretory granules and then by a process of maturation get concentrated in so called mature secretory granules, which are then released into the extracellular space under appropriate signal stimulation (for review see (Tooze et al., 2001)).

COPI coatomer and clathrin share at least one conserved structural element, implying that they operate by similar principles. But clathrin coats are considerably more complex than COPII and COPI. A variety of clathrin adaptors are recruited from cytosol to membranes and a specific combination of which form a heterogenous membrane-proximal layer onto which clathrin is subsequently deposited. Moreover, when compared to COPI and COPII coat mediated traffic, each and every step in clathrin coated vesicle mediated transport is regulated by an ensemble of kinases, phosphatases and other accessory proteins necessary to maintain the 
fidelity and specificity of multiple post-Golgi sorting events mediated by clathrin coats.

\subsection{Components of Clathrin-coated vesicle transport machinery}

At least ten different transport vesicle coats, and therefore classes of transport vesicle have been identified so far (Robinson and Bonifacino, 2001). One of the major classes of transport vesicles is the clathrin-coated vesicles (CCVs) and their role in internalization of proteins from PM by a process called clathrin mediated endocytosis(CME), is the most extensively studied mechanism of vesicle biogenesis. There are well over 20 proteins implicated in CCV assembly. Many interact with multiple partners creating a complicated network of possible interactions. A CCV is typically spherical and $60-100 \mathrm{~nm}$ in diameter. CCVs and clathrin-coated pits are considered as having a three-layered structure consisting of an inner layer of embedded transmembrane protein cargo linked to the outer clathrin layer by a middle layer of adaptor molecules. Other components of a CCV or clathrincoated pits include a large array of proteins with diverse functions, referred to as accessory proteins, which play distinct and dedicated roles in CCV formation, budding and fusion. Over the past decades, biochemical studies dissected most of the components of CCV transport machinery, while recent structural studies on clathrin and clathrin coat components has shed much light on the mechanism of clathrin coat mediated cargo transport at the molecular level.

\subsubsection{Clathrin}

As a main constituent of CCVs, clathrin polymerizes into polyhedral lattices to form the outer layer of a forming vesicle. It acts as a molecular scaffold to provide stability to the vesicle and also as a platform for the recruitment of a subset of accessory proteins. A Clathrin coat is a three-dimensional array of triskelia. Each triskelion unit consists of three (approx.,190-kDa) clathrin heavy chains(CHCs) and three 25-29-kDa clathrin light chains(CLCs). These triskelia polymerize into polyhedral lattices of regular shape, with one unit centered on every edge and its legs lining two sides of a polygon. The N-terminal domains of CHCs are facing inwards to establish contact with adaptor molecules that link clathrin to the membrane and its C-terminal domain accomodates CLCs. For a detailed review on the structural organisation of clathrin and clathrin coats, see (Kirchhausen, 2000; Edeling et al., 
2006).

Purified clathrin can spontaneously assemble into cages at slightly acidic $\mathrm{pH}$ (Kirchhausen and Harrison, 1981) or , in the presence of adaptor proteins, into coats in vitro. Clathrin coats can form different shapes and sizes. But under physiological buffer conditions, in the presence of adaptor molecules, clathrin baskets appear smaller and are of more regular and uniform size (Pearse and Robinson, 1984). Clathrin-coated pits at the PM and TGN are not static, but are dynamic structures. Using fluorescence recovery after photobleaching(FRAP), it was shown that both clathrin and adaptors at coated-pits, rapidly exchange with free proteins in the cytosol and the adaptors were able to exchange independently, even when clathrin exchange was blocked (Wu et al., 2003). In another study (Hinrichsen et al., 2006), it was shown using electron microscopy that at cellular cortices, upon depletion of clathrin, the membrane domains containing only the adaptor molecules lack any curvature suggesting that clathrin is necessary for driving coated-pit invagination and was proposed that clathrin could contribute for vesicle budding by recruiting membrane-bending proteins like epsin. Moreover, apart from playing a major role in vesicular trafficking, a recent study (Royle et al., 2005) demonstrated a novel and an unconventional role for clathrin in mitosis, wherein clathrin binds the mitotic spindle through its heavy-chain and stabilizes it .

\subsubsection{Adaptor proteins}

Intracellular vesicle transport is mediated by clathrin adaptor molecules which are classified as either multimeric or monomeric adaptors whose function is compartment specific as depicted in Fig. 1.2.

\subsubsection{Heterotetrameric Adaptor Protein complexes}

Two adaptor protein complexes, AP-1 and AP-2, were originally purified from clathrin-coated vesicles (Hirst and Robinson, 1998; Schu, 2001; Robinson and Bonifacino, 2001). According to their ability to promote the assembly of clathrin cages in vitro they were named assembly polypeptides or adaptins (Pearse and Robinson, 1984). AP-1 localises to the TGN and to endosomes, whereas AP-2 is exclusively found at the plasma membrane. Database searches have identified two more members of the family, AP-3 and AP-4, which are absent from clathrin-coated vesicle preparations. Although AP-3 was initially assigned to a perinuclear region like the TGN and to endosomal structures, recent studies demonstrate that it mainly present on endosomal tubules (Dell'Angelica et al., 1997; Simpson et al., 


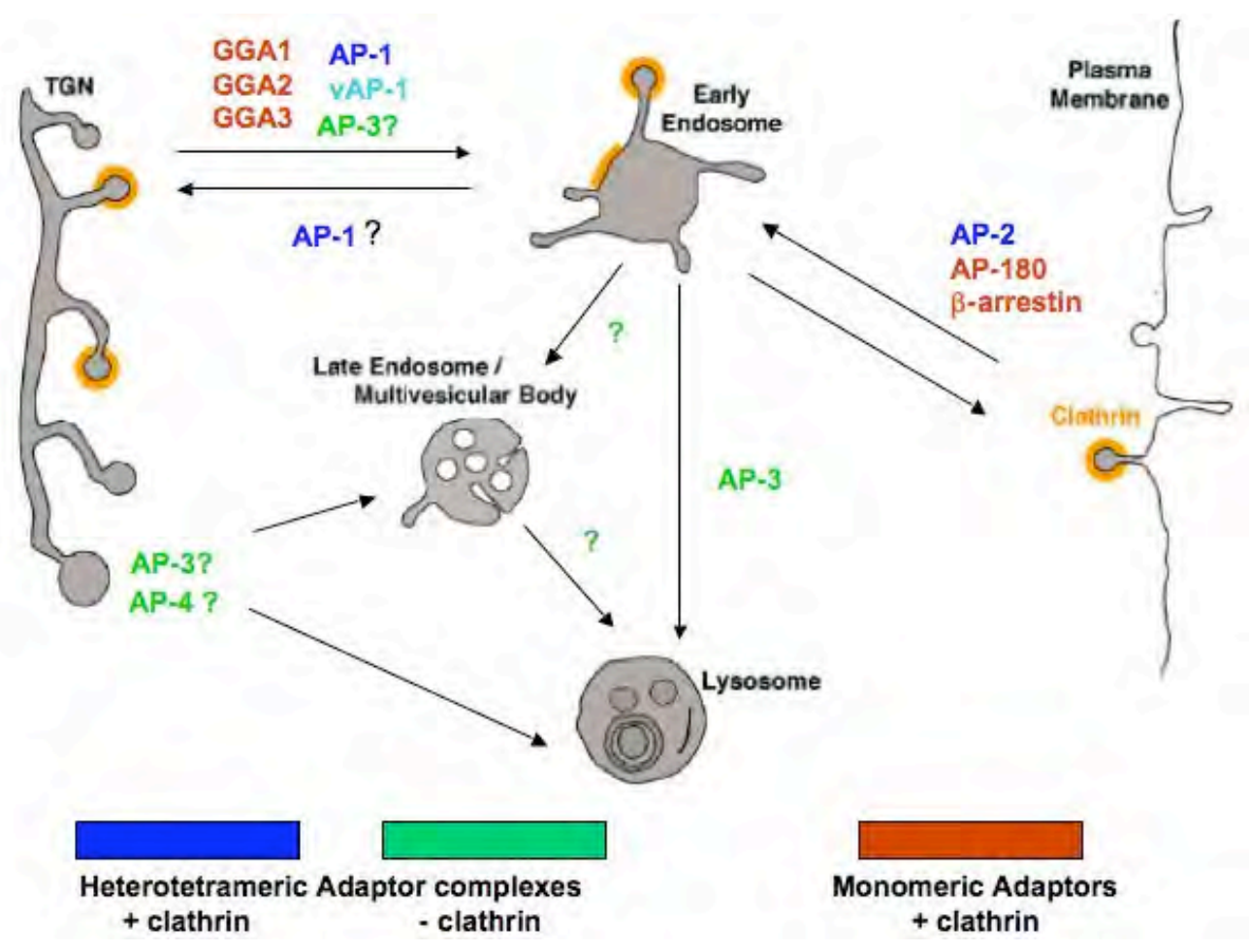

Figure 1.2: Compartment-specific role of Adaptor proteins.Different sets of heterotetrameric Adaptor Proteins (AP) operate vesicular transport either in Clathrin dependent (blue) or independent (green) fashion between specific routes. Also Monomeric Adaptors (red) perform overlapping functions along with AP complexes. Ambiguity of the role of adaptors in a certain pathway is indicated by a question mark.

1997; Peden et al., 2004). It was shown to interact and colocalise with clathrin, albeit to a lower extent compared to AP-1, suggesting a function as clathrin adaptor of lower affinity or different assembly properties (Dell'Angelica et al., 1998; Peden et al., 2004). In contrast, AP-4, which has been implicated in sorting at the TGN, obviously does not bind to clathrin (Simmen et al., 2002). All the adaptor complexes consist of four adaptin proteins. The two large subunits of about $100 \mathrm{kD}, \gamma / \alpha / \delta$ $/ \epsilon$ and $\beta$ 1-4 for AP1-4, respectively, fold into an amino-terminal trunk domain and a carboxyterminal ear or appendage domain that are connected by a largely unstructured hinge. The trunk domains together with the medium-sized subunits, $\mu 1-4$ (about $50 \mathrm{kD}$ ), and the small subunits $\sigma 1-4$ (about $20 \mathrm{kD}$ ) form the core complex. Between AP-1 and AP-2, $\beta$ is the most conserved subunit showing $84 \%$ sequence identity. Whereas the $\mu$ - and $\sigma$-subunits are about $40 \%$ identical, only $25 \%$ of the amino acids are conserved between $\gamma$ and $\alpha$, mostly due to the divergence of the ear domains. Despite the differences in primary sequence, predictions of secondary structure and hydrophilicity profiles indicate that all the four complexes are highly homologous. Comparison of the AP- 2 and AP- 1 crystal-structures confirms 
this prediction. Thus, the crystal structure of AP-2 can serve as a model for the entire family.
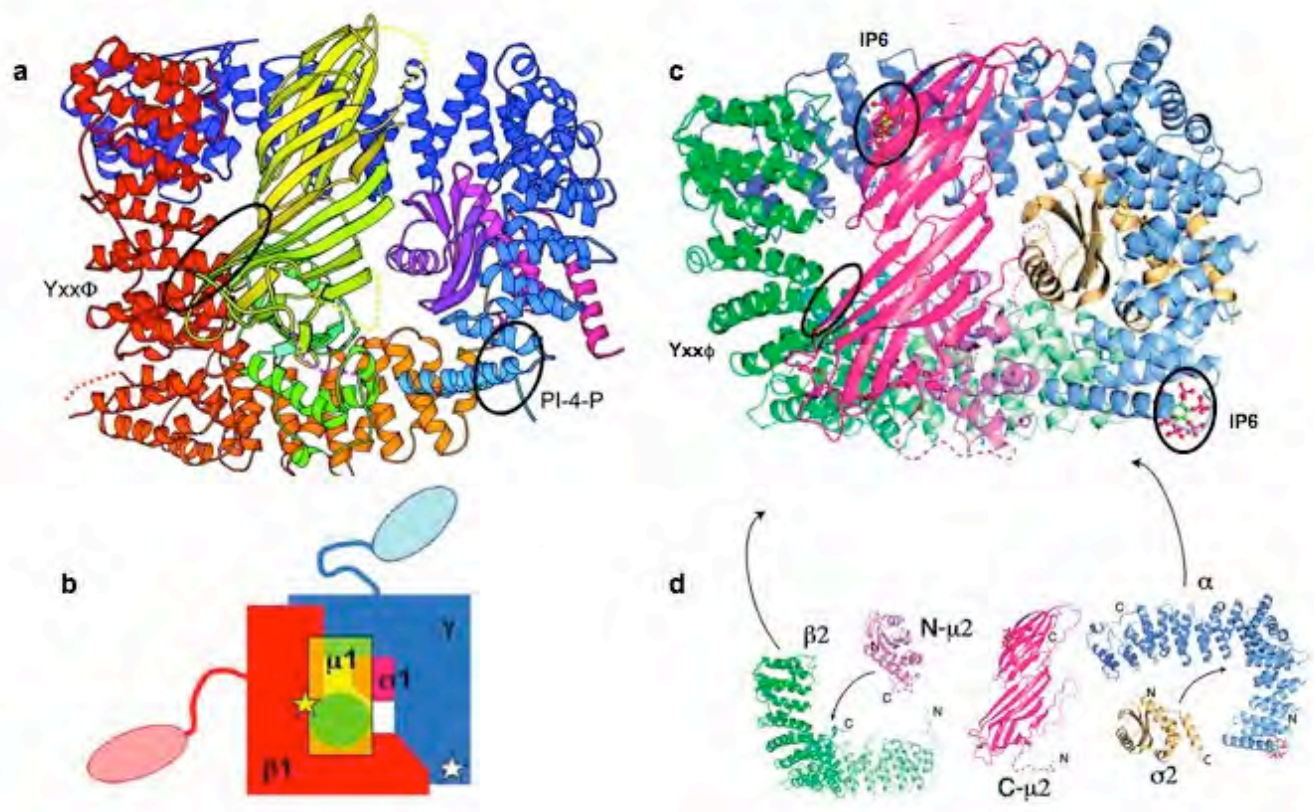

Figure 1.3: Structure and assembly of (a) AP-1 and (c) AP-2 core.Individual subunits are represented by respective colours in (b) and(d). The location of $Y \times x \phi$ binding site is indicated by black ellipse. Black circles represent PI-4-P binding site in AP-1 and D-myo-inositol-1,2,3,4,5,6hexakisphosphate(IP6) in AP-2. Note that AP-1(C- $\mu 1)$ lacks the phosphoinositide binding site compared to the corresponding site in AP2 (C- $\mu 2)$. (Heldwein et al., 2004, Collins et al., 2002)

The large subunits $\alpha$ and $\beta 2$ form curved arrays of $\alpha$-helices, which accommodate the globular $\sigma 2$ and N-terminal $\mu 2$-domains, respectively. $\sigma 2$ and N- $\mu 2$ both contain a central five-stranded $\beta$-sheet surrounded by $\alpha$-helices. $\mathrm{N}-\mu 2$ is connected to the all $\beta$-sheet by a 23-residue loop. All the subunits contact each other to some extent, but stronger interactions link $\alpha$ to $\sigma 2$ as well as $\beta 2$ to $\mu 2$. In fact, these two dimers show significant similarity on the amino acid level and their folds can be matched onto each other, particularly for the N-terminal domain of $\mu 2$ and $\sigma 2$ and for the amino-termini of the large subunits. $\sigma 2$ is related to the N-terminal domain of $\mu 2$ even at the primary sequence level. The AP- 2 core complex harbours two phosphoinositide binding sites, one in the $\mathrm{C}-\mu 2$ domain and one at the N-terminus of $\alpha$ ( Fig. 1.3). Sequence alignment of AP-2 and AP-1 counterparts indicates that these binding sites are basically conserved. Recently, the structure of AP-1 core complex was solved by X-ray crystallography (Heldwein et al., 2004) and it turned out to be that the overall structure was similar to AP-2, but significantly the AP-1(C$\mu 1$ ) lacks the phosphoinositide binding site compared to the corresponding site in 
AP2 (C- $\mu 2)$. The core complex measures about $100 \AA x 80 \AA$, the hinge regions of the two large subunits can probably stretch about 200-300 Åaway from the core, since they have no significant secondary structure. As a consequence, the ear domains can reach long distances facilitating interaction with other proteins.

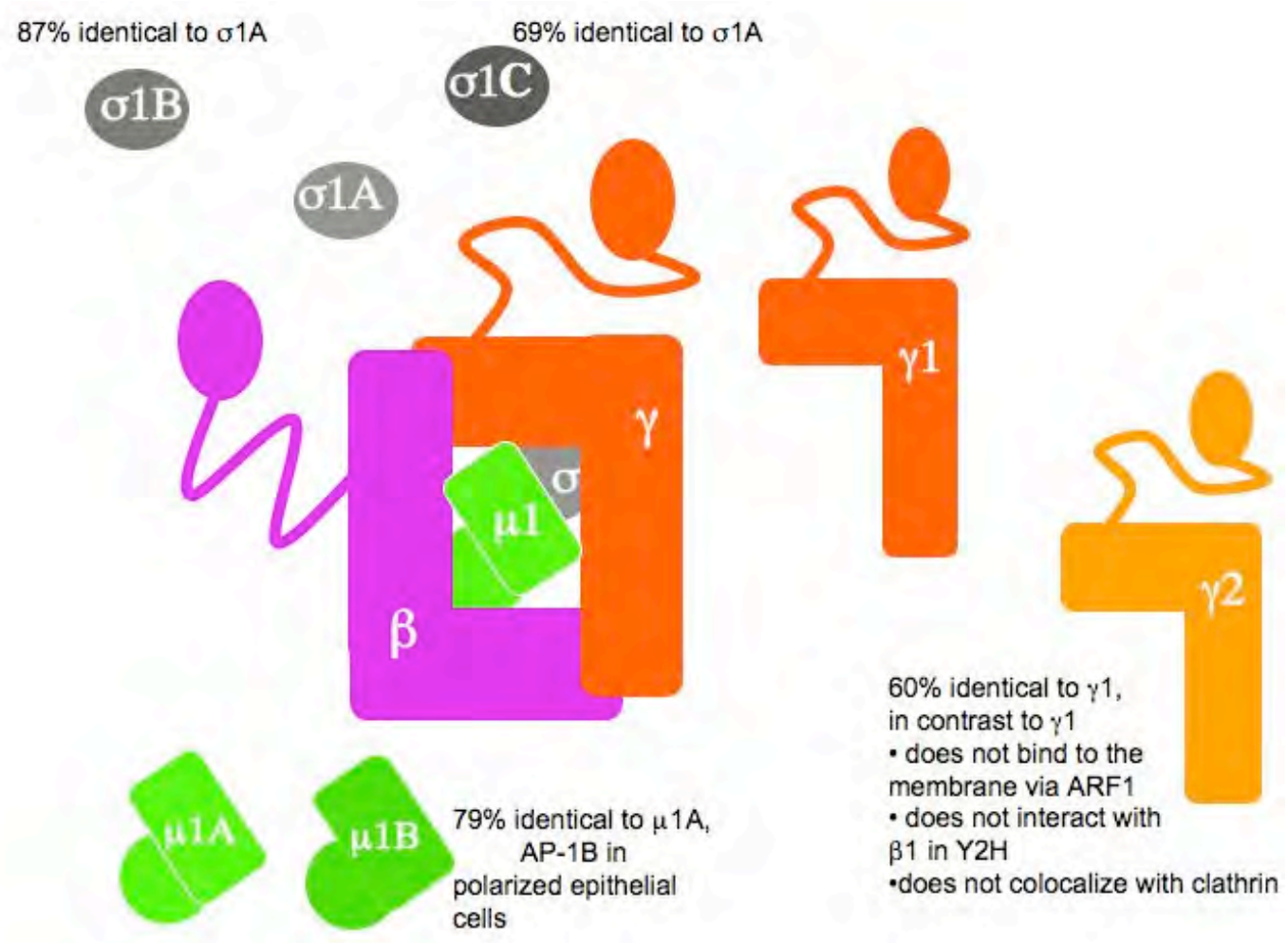

Figure 1.4: Schematic representation of variations in AP-1 subunit Isoforms. See text for details.

For many of the adaptor subunits, several genes are known in mammals. In the case of AP- 1 adaptor complex, except for the $\beta 1$ subunit, for all other three subunits at least one isoform is found to be expressed. The variations in the AP-1 subunit isoforms is schematically shown in Fig.1.4. $\gamma 2$-adaptin is about $60 \%$ identical to $\gamma 1$-adaptin, being shortened in the hinge region. In contrast to $\gamma 1$, it does not interact with $\beta 1$ in the yeast two-hybrid system, which indicates that they will not form a complex in vivo. Three genes are known for the $\sigma 1$-subunit, named $\sigma 1 \mathrm{~A}$, -B and -C that are about 70-80\% identical to each other. $\sigma 1 \mathrm{~A}$ and $\sigma 1 \mathrm{~B}$ apparently bind to both $\gamma 1$ and $\gamma 2$ in vitro (Takatsu et al., 1998, 2001). Moreover, a recent study of our lab, identified different splice variants for the $\sigma 1 \mathrm{~B}$ gene with tissue specific expression pattern which were designated as $\sigma 1 \mathrm{~B} 2$ and $\sigma 1 \mathrm{~B} 3$ and shown to be expressed mainly in the brain and skeletal muscle respectively (Riel, 2004). The significance of this tissue specific expression pattern is presently unknown. In 
addition to the ubiquitous subunit $\mu 1 \mathrm{~A}, \mu 1 \mathrm{~B}$ is selectively expressed in polarized cells and assembles into the variant complex AP-1B (Ohno et al., 1999; Fölsch et al., 1999; Eskelinen et al., 2002). $\alpha$-adaptin exists as two different proteins, $\alpha \mathrm{A}$ and $\alpha \mathrm{C}$, which are $80 \%$ identical (Ball et al., 1995). In addition to a ubiquitous alternative for $\sigma 3(\sigma 3 \mathrm{~B})$, neuron-specific variants are known for $\mu 3$-adaptin $(\mu 3 \mathrm{~B})$ and $\beta 3$ adaptin ( $\beta$-NAP) and they have been shown to form a variant neuronal AP-3B complex (Faúndez et al., 1998). No additional genes for AP-4 have been described.

\subsubsection{Monomeric Adaptor Proteins}

In addition to multimeric adaptor complexes, monomeric adaptor proteins were identified to exist, defined by their ability to recruit clathrin and to recognize the cargo for coated vesicle mediated transport. They are generally modular proteins, combining several conserved protein-protein or protein-lipid interaction domains.

The monomeric clathrin adaptors, which are referred to as alternate adaptors, that function at the PM, can be further classified based on their function either as cargo-specific adaptors (such as $\beta$-arrestins, ARH, Dab2 or numb) or phospholipid adaptors (proteins that belong to the epsin super family- epsin1, CALM/AP180, HIP1 and Hip1R). $\beta$-arrestins-1 and -2 recognize 7 -transmembrane helix GPCRs (G-protein coupled receptors) and mediate their rapid internalization and this is mediated by binding of $\beta$-arrestins to the PtdIns $(4,5) \mathrm{P}_{2}$ (Gaidarov and Keen, 1999), and the ability to bind physically to both clathrin and AP-2 (Claing et al., 2002; Marchese et al., 2003). ARH (autosomal recessive hypercholesteremia), Dab1/2 (Disabled1/2) and numb are clathrin adaptors involved in the uptake of the members of the LDLR family, by recognizing the sorting motif FxNPxY through their PTB (phospho-tyrosine binding) domain (Garcia et al., 2001; Morris et al., 2002; Santolini et al., 2000). Furthermore, the PTB domain of these proteins can bind to PtdIns(4,5) $\mathrm{P}_{2}$ and similar to $\beta$-arrestins, at their COOH-terminus, these proteins contain clathrin and AP-2-appendage binding sequences (Dho et al., 1999; Howell et al., 1999; Mishra et al., 2002a,b). A second group of putative alternate adaptors includes epsin 1, AP180/CALM and HIP1/Hip1R (Wendland, 2002). These proteins contain a structurally related $\mathrm{NH}_{2}$-terminal $\operatorname{PtdIns}(4,5) \mathrm{P}_{2^{-}}$ binding domain (the ENTH/ANTH domain) (Ford et al., 2001) and all bind to and colocalize with AP-2/clathrin. Epsins contain Ubiquitin interaction motifs (UIMs) and based on the role of yeast epsins, Ent1p and Ent2p which mediate endocytosis of ubiquitinated receptors, in mammals epsins may operate in internalization of ubiquitinated receptors, for example EGFRs. AP180 and HIP1/Hip1R lack these UIMs and in the case of AP180, an interaction with cargo proteins has not been 
demonstrated until now, suggesting that it serves mainly to stabilise and shape the coat. It is believed that these endocytic monomeric adaptors would probably function without AP-2 or alone, though not very efficient compared to the concerted activity (Hinrichsen et al., 2003; Motley et al., 2003). For example, AP180 promotes clathrin assembly onto a lipid monolayer in vitro and influences the size of coated vesicles in vivo (Zhang et al., 1998). However, AP-2 needs to participate to drive bud formation and the combination of AP180 and AP-2 works more efficiently in this respect than AP-2 alone. But a recent study has reported that Dab2 can sort LDLRs into coated-pits independent of AP-2 and ARH (Maurer and Cooper, 2006) which indicates that these monomeric adaptors are not just accessory proteins as previously thought, but can function as bonafide adaptors.
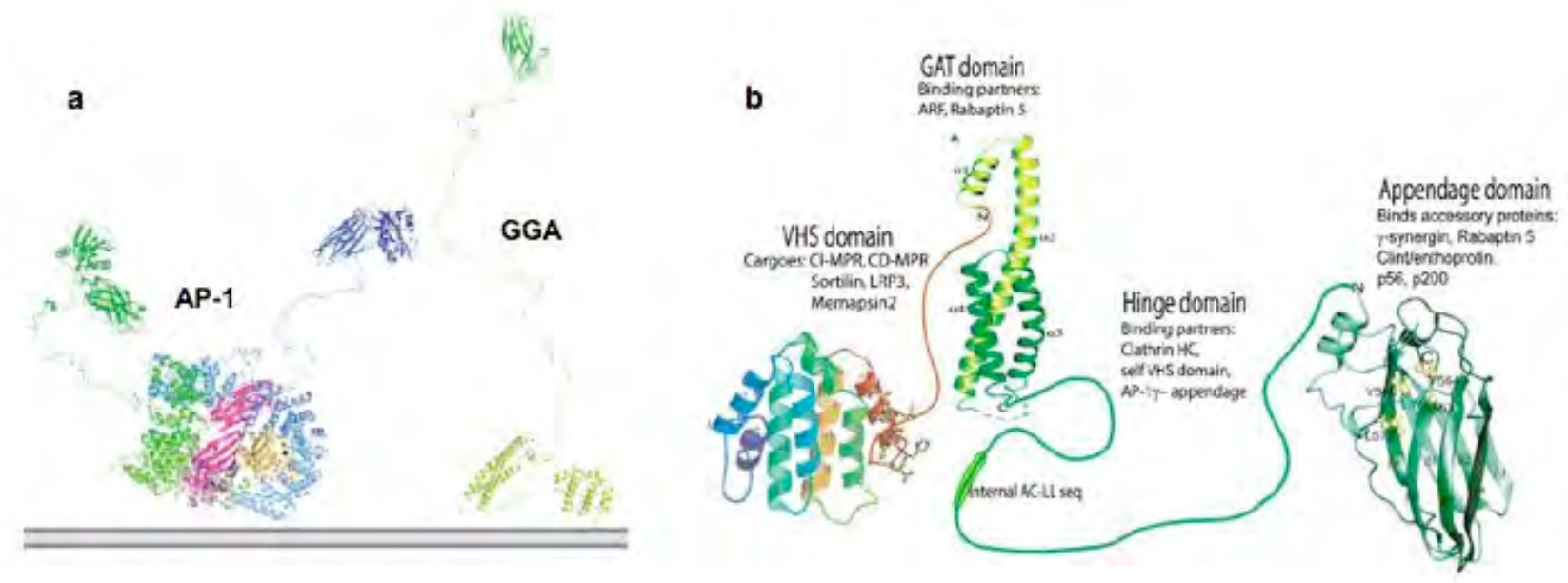

Figure 1.5: GGA1 in comparison to AP-1. (a) Structural models of AP-1 and GGA. (b) Model of the overall structure of GGA1. Different domains and their binding partners are shown. The VHS domain binds to the acidic-dileucine (AC-LL) motif present in the cargoes. Note that the Hinge domain harbors an Internal AC-LL motif which can mask the function of VHS domain by auto-inhibition and hence the sorting function of GGA1 can be regulated (Ghosh and Kornfeld, 2003b). There is no evidence that AP-1 has such a mode of regulation.

The GGA proteins were identified and extensively studied by several groups during the last few years in the context of sorting at the TGN (for rev. see (Bonifacino, 2004)). In addition, they localize to early endosomes. Three genes are known in mammals, encoding for GGA1, GGA2 and GGA3, and the family is conserved among eukaryotes as supported by two homologues in yeast. The GGA proteins consist of several domains that they share with other proteins: At the N-terminus they contain a common interaction module in vesicular traffic, the VHS domain (named after the first family members Vps27p, Hrs and STAM), followed by the GAT domain (which is also present in TOM1=target of Myb1), 
a rather unstructured linker and the C-terminal GAE ( $\gamma$-adaptin-ear-homologous) domain. These domains convey properties which are very similar to AP-1. By means of the GAT domain, GGAs bind to ARF1 and ARF3 which is sufficient for their targeting to the TGN. Sorting signals of the DxxLL type, including a cluster of acidic residues around the essential aspartate and and an upstream serine residue whose phosphorylation enhances sorting efficiency, are recognized by the VHS domain. Clathrin binds to clathrin box motifs in the linker region and to an additional site within GGA1-GAE. In analogy to the adaptins, the GAE domain accounts for recruitment of accessory proteins, displaying preferential affinity to p56, a protein of currently unknown function. Whereas p56 and $\gamma$-synergin bind to ear domains of both $\gamma 1$-adaptin and GGA in vitro, p56 colocalizes with GGA and $\gamma$-synergin with AP-1 in vivo and membrane association depends on the presence of GGA or AP-1, respectively (Lui et al., 2003). Rabaptin-5 associates with GGA proteins via the GAT and GAE domains and is involved in their endosomal targeting (Mattera et al., 2003). Despite the similarity of the three GGAs, they are not completely redundant: Crystal structures of the VHS domains revealed that GGA2 differs significantly from GGA1 and GGA3 such that it lacks a critical serine residue that is subject to phosphorylation-induced regulation of function of GGA1 and GGA2. In contrast to GGA2, the two latter proteins are regulated by phosphorylation which induces a conformational change. Phosphorylation of a serine residue adjacent to an internal acidic-cluster-dileucine motif in the linker region enables this motif to occupy the cargo binding site in the VHS domain. Since other interaction sites, namely for ARF and cargo proteins, become buried by this fold, this is considered to be the closed cytosolic form of GGA1 and GGA3. Membrane association probably occurs after dephosphorylation by PP2A, whereas phosphorylation can be mediated by CKII, a kinase associated with AP-1 (Doray et al., 2002; Ghosh and Kornfeld, 2003b). It was shown recently that all the GGAs colocalize in coated pits at the TGN and that their membrane-bound pools interact with each other via multiple domains. Depletion of any GGA protein resulted in increased degradation of the other two (Ghosh et al., 2003). Although GGA proteins exhibit the basic properties of adaptors and localize to clathrin-coated pits and/or CCVs by EM (Doray et al., 2002), they have not been detected in purified CCVs until now. They play an essential part during sorting events at the TGN and may cooperate with AP-1, but their function has not been clearly defined. Surprisingly, recent data demonstrate that GGAs bind to ubiquitinated proteins in vitro (Puertollano and Bonifacino, 2004). Ubiquitination of endocytosed proteins targets them to lysosomes for degradation. Cargo selection occurs at early endosomes and involves the ESCRT machinery as well as specialised clathrin-coated membrane patches initiated by the monomeric 
adaptor Hrs (Raiborg et al., 2001a). In contrast to other coated pits, this region does not generate coated vesicles, but probably clusters the components which should be present in the internal vesicles of a multivesicular body (Sachse et al., 2002). Like the GGA proteins, Hrs contains a VHS domain, followed by a FYVE module, which is a zinc finger fold that binds specifically to PI3P. A ubiquitin-interacting motif (UIM) serves to sequester ubiquitinated proteins destined for degradation in lysosomes. There are further two coiled-coil regions present and a functional clathrin box motif at the C-terminus. The endosomal localization of Hrs depends on a functional FYVE domain together with the coiled-coil domains (Raiborg et al., 2001b). All the GGA proteins were shown to interact with ubiquitin, but GGA3 displayed the highest affinity. Depletion of GGA3 by RNAi significantly delayed the degradation of EGFreceptor (EGFR) in lysosomes in addition to endosomal membrane reorganisation. As GGA3 binds to a subunit of the ESCRT complex as well, a role in ubiquitin sorting seems possible and has to be addressed by further experiments (Puertollano and Bonifacino, 2004)

\subsubsection{Accessory Proteins}

Apart from the clathrin adaptor molecules, discussed in the previous sections, a number of proteins are increasingly being identified which play necessary roles for clathrin-coated vesicle transport. Structural and biochemical studies have localized the binding sites of a plethora of accessory/regulatory proteins and other proteins called CLASPs (clathrin associated sorting proteins) to the appendage domains of adaptor proteins. These accessory/regulatory proteins act at distinct steps aiding clathrin-coat assembly formation, at downstream steps like inducing membrane curvature by recruiting actin cytoskeleton to membranes, at the step of vesicle scission by recruiting the GTPase-Dynamin and at steps involving the uncoating of CCV to be primed for fusion with the acceptor membrane.

BAR (Bin, amphiphysin, Rvs) and ENTH (Epsin N-terminal Homology) domains are families of $\alpha$-helical lipid bilayer binding modules found in proteins that function in endocytosis, actin regulation and signaling. Several members of these families not only bind the bilayer, but also participate in the regulation of its curvature. These properties are thought to play physiological roles at sites of membrane budding and at other sites where narrow tubular membranes occur in vivo. Studies of BAR and ENTH domains and of their flanking regions have provided new insights into mechanisms of membrane deformation and curvature sensing, and have emphasized the importance of amphipathic helices, thought to intercalate in one of the leaflets of the lipid bilayer, in the generation of membrane curvature (for 
review see (Itoh et al., 2001)). One of the widely studied BAR domain containing proteins are amphiphysin $1 / 2$ which contain $\mathrm{NH}_{2}$-terminal BAR domain followed by an unfolded region and a COOH-terminal SH3 domain. Their SH3 domains bind dynamin and synaptojanin, which are implicated in the fission and uncoating of endocytic vesicles respectively. The central region of the brain isoforms of amphiphysin comprises binding sites for the heavy chain of clathrin and for the clathrin adaptor AP-2 (Slepnev and De Camilli, 2000). Based on these findings, the amphiphysins were proposed to provide a functional link between clathrin-mediated budding, dynamin-dependent fission and the subsequent uncoating.

\subsubsection{Interaction of AP-1 with accessory Proteins}

The $\gamma 1$-ear domain contains a discontinuous motif of basic amino acids that was shown to interact with several proteins, like $\gamma$-synergin, Eps15, EpsinR, Rabaptin5, Snx9 and ARF1 GAP1 (Page et al., 1999; Kent et al., 2002; Hirst et al., 2003). Their respective binding site to $\gamma 1$-adaptin was found to conform more or less to the stretch DFxDF (Mills et al., 2003; Duncan et al., 2003). $\gamma$-synergin associates with cytosolic and membrane-bound AP-1 and is enriched in CCVs. Both a GTPase and AP-1 are necessary to recruit it onto the membrane. It further contains an 'Eps15-homology' (EH) domain, a common fold among endocytic proteins that binds to the tripeptidyl sequence motif 'NPF', suggesting a function as linker protein (Page et al., 1999; Hirst et al., 2003). Eps15 was mainly characterized in the context of clathrin-mediated endocytosis. Although it shows a BFA-sensitive perinuclear immunostaining, it remains unknown if Eps15 interacts with AP-1 in vivo (Kent et al., 2002). EpsinR (synonymous to Enthoprotin / Clint) belongs to a group of proteins that interact with phosphoinositides via their 'epsin N-terminal homology' or ENTH domain, in this case preferentially with PI4P (Mills et al., 2003; Hirst et al., 2003). Surprisingly, the crystal structure of the related epsin1 ENTH domain in the presence of phospholipid molecules visualised an additional amphipathic $\alpha$-helix compared to the lipid-free structure (Ford et al., 2002). When incubated with liposomes, this ENTH domain generated thin tubules. According to these observations, it was proposed that ENTH proteins can insert an amphipathic helix into the membrane and thereby promote membrane curvature. Even if epsinR seems to bind to lipids with less affinity, the versatile helix region is well conserved. Similar to $\gamma$-synergin, epsinR is enriched in CCVs and localizes to the membrane in an ARF-dependent manner, but it does not require AP-1 for membrane binding. Moreover, it directly interacts with clathrin (Mills et al., 2003) and the SNARE protein Vti1b (Chidambaram et al., 2004), supporting a role as tethering and 
stabilising factor in coat formation. Rabaptin-5, which binds to the ear domains of $\gamma 1$ and $\gamma 2$ in vitro, functions as a divalent linker between Rab4 and Rab5, GTPases involved in endosomal trafficking (Stenmark et al., 1995). It has been shown to interact with a dimeric $\gamma 1$ - $\sigma 1$-adaptin complex on endosomes and to connect it to Rab4 (Deneka et al., 2003). However, it is not known whether this occurs during coat formation or at a later stage of transport. As for ARF1 GAP and the sorting nexin 9 (Snx9), their physiological role as AP-1 binding partners remains to be demonstrated. Recently, a novel protein called $\gamma$-BAR ( $\gamma 1$-adaptin Brefeldin A Resistance) was found to interact with the $\gamma 1$-ear-domain but not with the highly homologous GAE domain of GGA1 and participate in the regulation of AP-1 membrane binding dynamics (Neubrand et al., 2005). Also, other accessory proteins like aftiphilin and NECAP (adaptin-ear-binding coat-associated protein) 1 and 2 were shown to bind to both $\gamma 1$-ear-domain and the GAE domain of GGA1 and also $\alpha$-ear-domain but with different affinities. It is speculated that this difference in affinities could be contributed by acidic residues that lie downstream of the identified interaction motifs WVQF and WGDF of these accessory proteins (Mattera et al., 2004).

\subsection{Life-cycle of Clathrin Coated Vesicles(CCVs)}

The mechanism of clathrin-coated vesicle formation and the events that follow thereafter have been extensively studied for AP-2 in the synaptic vesicle recycling and endocytic pathway. There is a significant variation in this mechanism at the TGN. It has been shown in vitro that adaptors bind to the terminal domain of clathrin and addition of $\mathrm{N}$-terminal fragment of the $\beta 2$-chain, comprising the hinge and the ear domain, will stimulate clathrin assembly (Shih et al., 1995). The entire process of clathrin lattice assembly has been reconstituted in an in vitro assay with fragments of clathrin and adaptors (Greene et al., 2000). The presence of two binding sites in one $\beta$-chain suggests that adaptors could potentially crosslink two CHCs to orient them in a conformation favourable for assembly. It has also been shown that phosphorylation of the $\beta$-chains plays an important regulatory role in CCV formation (Ghosh and Kornfeld, 2003b; Wilde and Brodsky, 1996). The hinge region of the $\beta$-chains is phosphorylated in the cytosol and hence cannot bind clathrin. When membrane-bound, $\beta$-chains are dephosphorylated and can interact with clathrin to stimulate assembly. However, the kinetics of phosphorylationdephosphorylation cycles with respect to the CCV formation is not well understood.

Initiation of the CCV formation occurs by the inward deformation of a flat array 
as a result of massive membrane reorganization (see Fig. 1.6, step 1). The factor that determines the location and initiation of coat assembly remains unknown. Adaptors serve to link clathrin to the membrane and to coordinate the structural assembly of the coat with the selection of cargo proteins and lipids. A number of proteins, in addition to the adaptors, contain clathrin-box sequences and any of these in principle could recruit clathrin. In addition to a clathrin scaffold, a membrane, cargo, and cargo adaptors, coated vesicles must include molecules that determine the destination and ultimate fusion of the vesicle. The identities of these targeting and fusion molecules are largely undetermined. They probably include soluble Nethylmaleimide sensitive factor (NSF) attachment protein receptors (SNAREs) and Rab proteins.

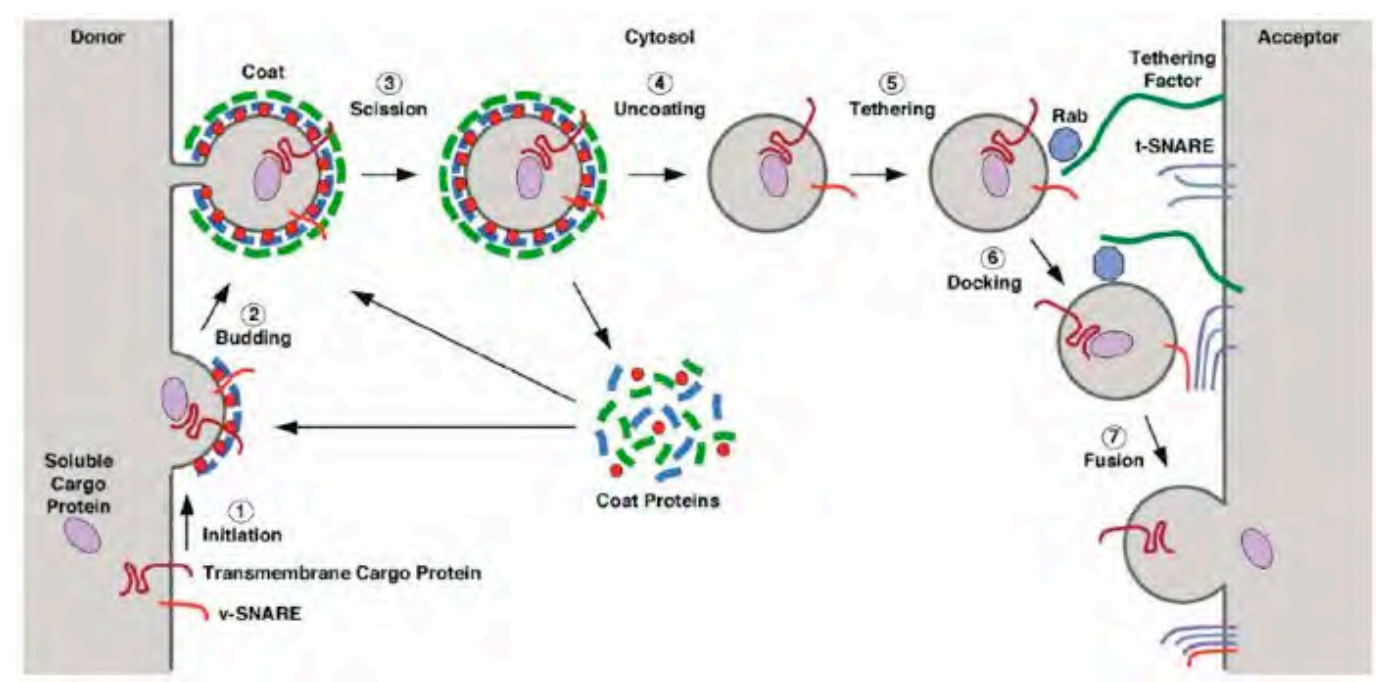

Figure 1.6: Steps in the life cycle of a coated vesicle.This scheme represents the steps involved in the Budding and Fusion of a coated vesicle. Formation of a coated vesicle at the donor membrane initiates by recruitment of membrane-proximal coat components (blue) to a membrane-associated GTPase (red) and/or to a specific phosphoinositide (1). Membrane-distal components (blue) are added and as the membrane curvature increases, the vesicle is severed $(2 \& 3)$. When the coated vesicle reaches the acceptor membrane, coat proteins are shed (4) and the coat proteins are recycled for next rounds of vesicle budding. The naked vesicle then tethers to the acceptor membrane guided by GTP-Rab and tethering factors; primed for fusion aided by $\mathrm{v}$ - and $\mathrm{t}$-SNARES complex formation $(5 \& 6)$. Fusion between the vesicle and acceptor membrane bilayer is promoted by this SNARE complex and the cargo transferred to the acceptor compartment (7). (from (Bonifacino and Glick, 2004)).

Once the growing buds (see Fig.1.6, step 2 and 3) at the sites of assembly are completely formed, they need to detach from the donor membrane as a vesicle and travel a certain distance in order to deliver their contents to the target organelle. Detachment of the coated vesicles from the plasma membrane requires the activity of a GTPase, dynamin (Hinshaw and Schmid, 1995). Two of the proteins recruited 
to a dynamin proline-rich region through their SH3 domains are amphiphysin and endophilin. Amphiphysin in turn interacts with synaptojanin. Synaptojanin is an inositol 5-phosphatase. Endophilin has lysophosphatidic acid acyltransferase activity (Huttner and Schmidt, 2000). This reaction generates phosphatidic acid potentially causing a change in membrane curvature and contributing to budding and scission. The activity of synaptojanin would favour release of dynamin by dephosphorylation of the head groups that recruit it. This suggests that PIP binding is a critical interaction stabilizing an assembled coat and that a inositide-specific phospholipase may play a role in vesicle uncoating (Cremona et al., 1999). Both isoforms of dynamin (Dynamin 1 and 2) have been shown to be involved in the formation of transport vesicles at the TGN hinting at similarities in the vesicle scission mechanism with plasma membrane CCVs.

The uncoating of the CCVs (fig. 1.6, step 4) is facilitated by the dissociation of the coat proteins from lipid bilayers of the membranes. Hsc70 is an ATPdependent chaperone, which binds relatively hydrophobic peptides or exposed protein sequences. It is involved in modulating the folding state of intracellular proteins for their translocation across ER, mitochondrial and lysosomal membranes. ATP hydrolysis by hsc70 is required for removing the clathrin triskelion from the vesicle and ADP stabilizes the hsc70- clathrin complex that is released (Prasad et al., 1994). Hsc70 proteins are drawn to specific targets by proteins that contain $\mathrm{J}$ domains (named after DnaJ, the partner of the E.coli Hsp70, DnaK). The coated vesicle protein Auxilin contains such a $\mathrm{J}$ domain. The recently defined role in cellular CCV disassembly for auxilin also strengthens the implication of hsc70 as a regulator of clathrin disassembly (Lemmon, 2001; Ungewickell et al., 1995). The phosphatase activity associated with auxilins could conceivably destabilize coat protein-PIP interaction. The recruitment of hsc70 to CCVs by auxilin followed by ATP binding and hydrolysis by hsc70 may cause a conformational change in assembled clathrin, triggering disassembly. It is not clear how lipid dissociation processes are coupled to disassembly of the clathrin lattice. Hsc70 releases clathrin, but not adaptors, from the isolated coated vesicles, which hinted that clathrin and adaptor dissociation are separate events. It was indeed shown that clathrin and adaptors dissociate from CCVs in separate steps (Hannan et al., 1998), but very little is known about how adaptors are released from the membranes. The regulatory events involved in sensing the completion of formation of the vesicle and initiating the uncoating reaction still remain a puzzle. Earlier studies had reported the requirement of a cytosolic factor in the uncoating process at the TGN which was also shown to require ATP and Hsc70 (Hannan et al., 1998). Recently it was shown that protein phosphatase 2A (PP2A) treatment of phosphorylated AP-1 from 
CCVs abolished binding to the tail peptides and released adaptors from CCVs to the cytosol. Hence PP2A was shown to be the cytosolic co-factor required in adaptor uncoating of CCVs at the TGN (Ghosh and Kornfeld, 2003a).

Some of the proteins, that can classify as cargo proteins during vesicle formation, belong to the transport machinery themselves. Rab proteins, another family of small GTPases, link the vesicles to the cytoskeleton or manage their correct docking at the target membrane. SNARE proteins mediate membrane fusion in the last step of transport (for rev. see (Jahn et al., 2003)). As assumed for all membrane fusion events, SNARE proteins reside on both the vesicle and the target membrane. Specific docking of the vesicle depends on the formation of the correct SNARE complex, which also approaches the two membrane surfaces to prepare for membrane fusion.

\subsection{Sorting signals}

The adaptor molecules bind to at least two different types of sorting signals in the tail of cargo. At least three types of sorting signals have been characterized in the context of AP mediated trafficking (for a detailed review, see (Bonifacino and Traub, 2003)).

\subsubsection{Tyrosine-based motifs}

Tyr-based motifs of the consensus $\operatorname{Yxx} \phi$ (x for any amino acid and $\phi$ for a bulky hydrophobic residue) are selectively recognized by the medium subunits. Cocrystallisation of the $\mu$-adaptin C-terminal domain with peptides containing a Tyrmotif identified the precise interaction site (Owen and Evans, 1998). Yxx $\phi$ motifs function in various transport pathways including endocytosis, lysosomal targeting or sorting to the basolateral plasma membrane (Marks et al., 1996; Bonifacino and Traub, 2003). Consistently, all the four $\mu$-adaptins bind to $\operatorname{Yxx} \phi$ sequences in vitro with distinct preferences for specific motifs (Ohno et al., 1998). The surrounding residues obviously contribute to this specificity as well as the exact spacing of the motif within the cytoplasmic domain of the protein. The motifs that were identified so far to be necessary for endocytosis are found in the middle of the cytoplasmic domain far from the transmembrane domain, but not at the Cterminus of the protein. In contrast, motifs for lysosomal targeting are often located close to the C-terminus. As shown for the lysosomal protein Lamp-1, changing the distance of the GYQTI signal from the membrane interfered with sorting to lysosomes, but not with internalization from the plasma membrane (Rohrer et al., 1996). Another Tyr-based motif with the consensus FxNPxY functions exclusively 
in clathrin- mediated endocytosis. Peptides comprising this signal can interact with the N-terminal domain of clathrin heavy chain as well as with purified AP-2 in vitro, but it is still unclear if these mediate the sorting in vivo. Moreover, monomeric proteins like ARH, Dab2 and numb, which were initially termed accessory proteins and were initially known for recognition of phosphotyrosine residues and implicated in downstream signalling of tyrosine-kinase receptors, show a remarkable affinity for unphosphorylated FxNPxY and are localized in coated-pits through interactions with AP-2, phospholipids and clathrin.

\subsubsection{Leu-based motifs}

Leu-based motifs were identified as sorting determinants from the plasma membrane and from the TGN to endosomes and lysosomes. Apparently, there are two different classes. (D,E)xxxL(L,I) signals, like $\mathrm{Yxx} \phi$, bind to adaptor complexes with preference for specific complexes and are found close to transmembrane domain as well as to the C-terminus. The acidic residue at the first position is not strictly required although favoured. Competition experiments with Tyr- and Leubased motifs demonstrated that they are recognized by independent binding sites (Marks et al., 1996). Until now, several subunits have been proposed to contain this site, namely $\mu$, as inferred from several in vitro studies, and $\beta$, which was crosslinked to peptides (Rapoport et al., 1998; Hofmann et al., 1999). Recently, Y3H experiments reported an interaction between $(\mathrm{D}, \mathrm{E}) \mathrm{xxxL}(\mathrm{L}, \mathrm{I})$ signals and a $\gamma 1$ $\sigma 1$ - or $\delta$ - $\sigma 3$-dimer, whereas none of the AP-1 or AP-3 subunits alone bound the motif (Janvier et al., 2003). The other class of Leu-based signals, DxxLL seems to be required for transport between TGN and endosomes. Because the leucines are generally preceeded by several acidic residues, these motifs are also named acidiccluster di-leucine motifs. In this case, the D residue cannot be replaced. In contrast to $(\mathrm{D}, \mathrm{E}) \mathrm{xxxL}(\mathrm{L}, \mathrm{I})$ signals, DxxLL does not bind to AP complexes in vitro, but to the monomeric adaptor group of GGA proteins (Zhu et al., 2001; Puertollano et al., 2001). Another family of sorting motifs consists of a stretch of acidic amino acids containing one to three phosphorylation sites for casein kinase II (CKII). This motif is present in a number of transmembrane proteins that cycle between the TGN and endosomes, and phosphorylation of the CKII sites is particularly required for retrieval from endosomes to TGN. A protein called PACS-1 (phosphofurin acidic cluster sorting protein 1 ) was shown to bind to phosphorylated acidic clusters, as well as to the adaptor complexes AP-1 and AP-3. Remarkably, the binding interface to AP-1 was mapped to $\mu 1$ and $\sigma 1$ (Wan et al., 1998; Crump et al., 2001). 


\subsection{Protein sorting at the TGN and endosomes}

A number of cargo proteins have been shown to interact with AP-1 at the TGN and are transported from the TGN to the endosomes by AP-1-mediated clathrin coated vesicle assembly. Most prominent among them are the MPRs (Hille Rehfeld, 1995), lysosomal membrane glycoproteins (LGP85 or LIMPII), invariant chain of MHC class II complexes (Rodionov and Bakke, 1998), furin (Molloy et al., 1999), gp48 (Reusch et al., 1999), HIV-1 Nef (Erdtmann et al., 2000). MPRs are the best known example of the transmembrane proteins sorted into the clathrin coats found on the TGN. They are selectively sorted at the TGN and exit this compartment in AP-1 containing clathrin-coated vesicles. MPRs are significantly enriched in purified clathrin-coated vesicle preparations (Borgne and Hoflack, 1997). Electron microscopic analysis have shown that both the MPRs and AP-1 or clathrin colocalize on budding profiles at the TGN (Klumperman et al., 1993, 1998). There is a direct interaction between the sorting signals in cytosolic domain of the MPR300 and AP-1 (Höning et al., 1997). AP-1-mediated sorting ensures that newly synthesized lysosomal hydrolases, bound to MPRs, are segregated from the molecules destined for constitutive transport to the plasma membrane and are delivered efficiently to the lysosomes via an intracellular route. However, since the GGA-proteins appear to have the same properties, the different functions of these two adaptor proteins in TGN-to-endosome sorting is not clear.

\subsubsection{AP-1 Versus GGA adaptors at the TGN sorting station}

For a long time, AP-1-containing-clathrin-coated vesicles were thought to form at the TGN and transport mannose-6- phosphate receptors (MPRs), the sorting receptors for lysosomal hydrolases that contain mannose-6-phosphate residues (Hille Rehfeld, 1995), to endosomes. The trafficking of MPRs in the $\mu 1 \mathrm{~A}$ 'knockout mice' (Meyer et al., 2000) provided the first evidence for a role of AP-1 in the retrograde transport from endosomes to the TGN. If AP-1 mediates anterograde transport from the TGN to endosomes, one would expect that in AP-1 deficient cells, the MPRs would get stuck in the TGN. But surprisingly, the MPRs exit the Golgi, get transported to the plasma membrane and are re-endocytosed, finally accumulating in an early endosomal compartment that contains the early endosome marker EEA1. This indicated that AP-1 might mediate not anterograde, but retrograde, transport between endosomes and TGN. The observation that Shiga toxin co-localizes with AP-1 on early/recycling endosomes during a $20{ }^{\circ} \mathrm{C}$ block of retrograde transport and that toxin transport is inhibited by BFA supports this idea (Mallard et al., 1998). 
This model gained further support when it was shown that the recently discovered GGA proteins mediate anterograde transport of MPRs and other transmembrane proteins that have acidic di-leucine motifs (Puertollano et al., 2001). However other studies showed by fluorescent tagged AP-1 that vesicular or tubular structures containing AP-1 leave the TGN and reach peripheral structures (Huang et al., 2001; Waguri et al., 2003), supports the idea that AP-1 is indeed involved in anterograde transport too.

When the N-terminal portion of a GGA protein that has the VHS and GAT domains, but lacks the clathrin-binding hinge domain and the GAE domain is expressed in mammalian cells, both MPRs accumulate in the TGN and clathrin is no longer detected on TGN membranes (Puertollano et al., 2001). AP- 1 localization is unaltered if the expression of the GGA N-terminus is kept at moderate levels. These data suggest that GGA proteins mediate clathrin-dependent anterograde transport of MPRs from the TGN to endosomes, a function long attributed to AP-1. So far, however, in mammalian cells there is no evidence that GGA proteins are a stable component of clathrin-coated vesicles; instead they redistribute very quickly to the cytoplasm under conditions where AP-1 stays on the membrane (Hirst et al., 2001). Subsequently, another study showed that the GGA hinge region binds to the $\gamma$-ear of AP- 1. This indicates that AP-1 and GGA proteins might interact and cooperate in the same sorting step (Doray et al., 2002). In immunoelectron microscopic studies using cells stably transfected with tagged GGA2, the authors demonstrate colocalization of GGA2 and AP-1 on coated buds of the TGN. Moreover, mutant MPR that does not bind GGA proteins fails to enter AP-1-coated vesicles.This let to the idea that GGA proteins are not necessarily packaged into vesicles but rather help recruit coat components and cargo into a budding vesicle. Further, the finding that the GGAs 1 and 3 are regulated by phosphorylation leading to an auto-inhibited state led to a hypothesis (Fig. 1.7) where GGAs were shown as adaptors that help to recruit cargo molecules into AP-1 conatining clathrin-coated vesicles. However, this model does not apply to GGA2, as it lacks the critical serine residue which was shown to be subjected to phosphorylation and dephosphorylation cycles. Altogether, the precise role of AP-1 and the GGAs is still not clear. 

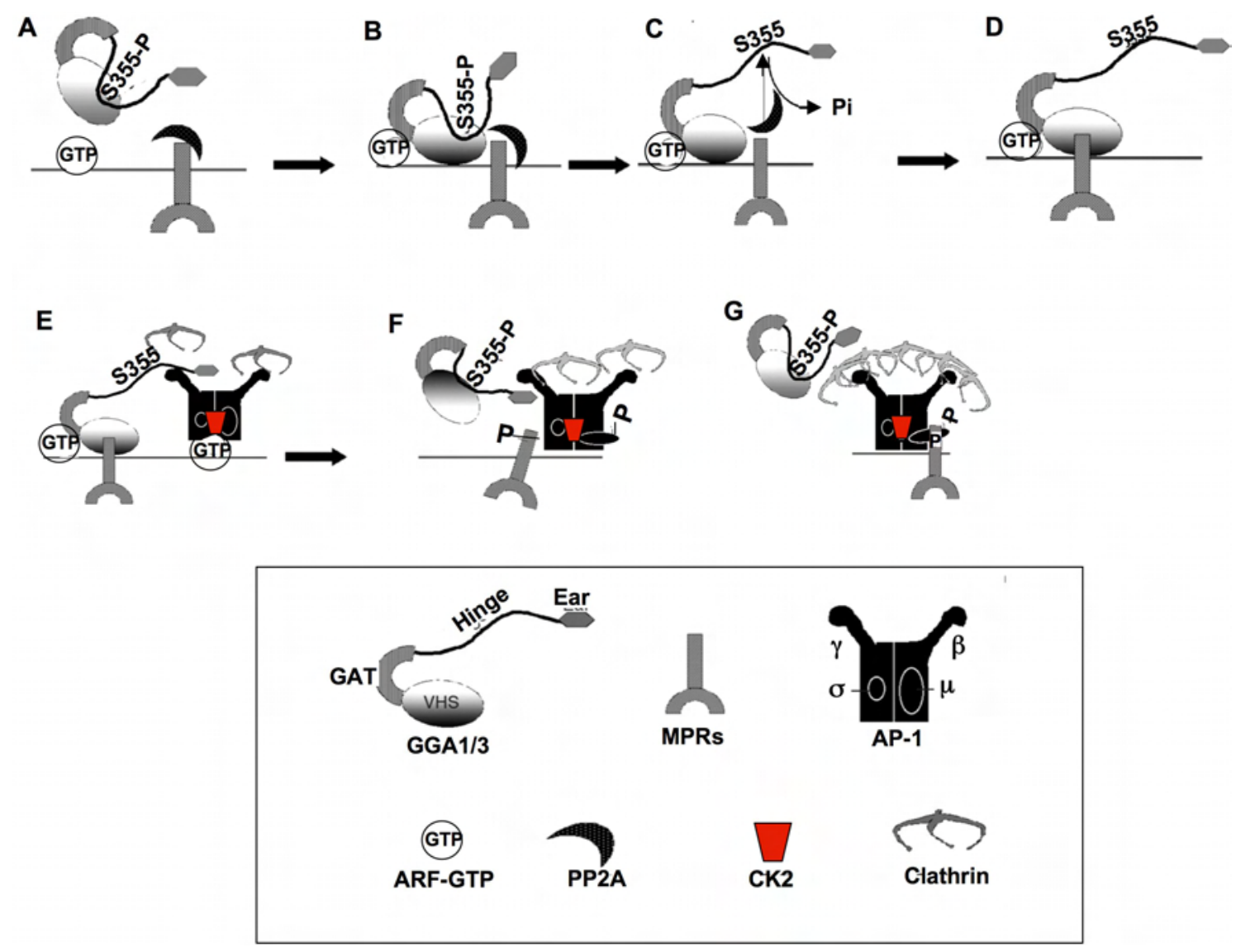

Figure 1.7: A model for Phosphoregulation of GGAs 1 and 3. (A) Cytosolic GGA in phosphorylation induced auto-inhibited state. (B) GGAs bind to membrane through ARF-GTP. (C) PP2A dephosphorylates GGA and relieves it from auto-inhibition. (D) The open form is now capable of binding to the cytoplasmic tail AC-LL motif of MPR. (E) GGAs can interact with $\gamma$-appendage domain of AP-1 on membranes via its hinge domain. (F) AP-1 associated CK2 phosphorylates GGAs and restores the auto-inhibited state. CK2 also phosphorylates the MPR tail. The phosphroylation of AP-1 $\mu 1 \mathrm{~A}$ at some point on membrane recruitment induces a open conformation of $\mu 1 \mathrm{~A}$, exposing ligand binding sites. This phosphorylation of MPR and of $\mu 1 \mathrm{~A}$ generates high avidity binding of MPR tails to AP-1, thereby ensuring directed transfer of cargo from GGAs 1 and 3 to membrane associated AP-1. (G) Thus MPR cargo molecules are trapped by AP-1 and as the clathrin coat assembly proceeds the auto-inhibited GGAs dissociate from AP-1 and are released to cytosol. (from (Ghosh and Kornfeld, 2003b)) 


\subsection{Aim of the study}

In the course of her Ph.D project in our group, C.Riel analyzed the expression pattern of $\sigma 1$-adaptin isoforms A,B and C in mouse tissues, each of which is encoded by an individual gene. In addition, she identified tissue specific variants of $\sigma 1 \mathrm{~B}$ adaptin (C.Riel and P.Schu, unpublished). $\mu 1$ adaptins and $\gamma 1-\sigma 1$ hemicomplex are able to bind to different sorting motifs in the cytoplasmic tails of cargo proteins. Sortilin receptor belongs to the family of Vps10p-domain receptors and it has been shown that it is sorted similar to the well studied mannose-6-phosphate receptors. Sortilin has been shown to be able to mediate lysosomal sorting, however it has also essential functions at the plasma membrane and internal organelles via heterodimerization. It has been reported that sortilin is sorted by GGA2, however nothing is known about AP-1-dependence of sortilin trafficking.

In a second project, the mechanism of AP-1 membrane binding should be studied. To identify possible function(s) of different domains of $\mu 1 \mathrm{~A}$-adaptin, a $\mu 2 / \mu 1$ chimera was constructed which was shown to assemble into a functional chimeric AP-1 complex, designated as AP-1*. But this AP-1* complex, was unable to restore the phenotype of $\mu 1 \mathrm{~A}$-deficient cells. During his Ph.D thesis project, Medigeshi R. Guruprasad discovered that the N-terminal seventy amino acids of $\mu 1 \mathrm{~A}$ are required for proper membrane-cytoplasm recycling of AP-1 and that impairing recycling causes mis-sorting of MPRs as indicated by secretion of their ligand cathepsin D into the culture medium (Guruprasad Medigeshi and P. Schu, unpublished). Therefore membrane binding of AP- $1^{*}$ should be analyzed in more detail. In addition, proteins binding to the $\mathrm{N}$-terminal seventy amino acids of $\mu 1 \mathrm{~A}$ were to be identified. 


\section{Chapter 2}

\section{Materials and Methods}

\subsection{Materials}

\subsubsection{Laboratory equipment}

Analytic balances type $1602 \mathrm{MP}$ and $1265 \mathrm{MP}$

Intelligent Dark Box II, Las-1000+

Ice machine

Centrifuges:

Eppendorf centrifuge Type 5415C and 5402

Table ultracentrifuge TL-100

Ulracentrifuge L8-70M

Cold Centrifuge J-21C and J2-MC

Labofuge GL

Rotors:

JA10, JA 20

Ti 45, Ti 60, Ti 70

TLA 45,

TLA-100.3

Electrophoresis chambers for agarose gels

Electrophoresis chambers for polyacrilamide gels

Gel dryer

Magnetic mixer

Microwave oven

pH-Meter

Photometer, UV 160 A

UV-hand lamp (365/254nm), Type 5415 and 5402
Sartorius, Goettingen

Fuji, Japan

Ziegra, Isernhagen

Eppendorf, Hamburg

Beckmann, München

Beckmann, München

Beckmann, München

Heraeus Sepatech

Beckmann, München

Beckmann, München

Beckmann, München

Beckmann, München

Workshop of the Institute

Workshop of the Institute

Bio-Rad, Hilden

IKA, Works, INC.

Siemens, München

Beckmann, München

Shimadzu, Kioto/Japan

Eppendorf, Hamburg 
Vacuum concentrator model $100 \mathrm{H}$

Vortex-Genie

DNA-Sequencer Type 310

Electroporator 1000 (used for bacteria)

Confocal Laser Scanning Microscope

Leica TCS SP2 AOBS

(Ar: 488, 514 nm; He/Ne: 543 nm; 63x Oil Objective)

Incubators Innova 4230 and 4330

Phosphoimager Fujix BAS1000

Ultra turrax T8

Supersignal Chemiluminiscent Substrate

Thermocycler GeneAmp PCR system 9600
Bachhofer, Reutlingen

Scientific Industries, USA.

ABI, PE Biosystems

Stratagene, USA

Leica, Bensheim

\subsubsection{Chemicals, plasticware and membranes}

Chemicals

Cell culture plasticware

Nitrocellulose membrane

PVDF membrane, $0.2 \mu \mathrm{M}$

Hybond-N Nylon membrane

Whatman GB002 paper

Whatman GB003 paper extra thick
Boehringer/Roche, Mannheim, Merck, Darmstadt, Roth, Karlsruhe, Serva, Heidelberg, Sigma, Deisenhofen Greiner, Frickenhausen Nalge Nunc International, Denmark Schleich and Schüll, Dassel Schleich and Schüll, Dassel Amersham Biosciences, UK Schleich and Schüll, Dassel Schleich and Schüll, Dassel

\subsubsection{Kits, spin columns and reagents}

\subsubsection{DNA, RNA}

HiSpeed Plasmid Midi kit

Omniscript Reverse Transcription kit

PCR purification kit

QIAprep Spin Miniprep kit

QIAquick Gel Extraction kit

Rapid-hyb hybridisation buffer
Qiagen

Qiagen

Qiagen

Qiagen

Qiagen

Amersham Pharmacia Biotech 
rediprimeTMII labeling system

RNAlater RNA Stabilisation Reagent

RNase inhibitor RNase Out

RNeasy Mini and Midi Kit

TA Cloning Kit

Effectene Transfection kit

Fugene Transfection reagent

Lipofectamine 2000

MATra transfection reagent
Amersham Pharmacia Biotech

Qiagen

Invitrogen

Qiagen

Invitrogen

Qiagen

Roche

Invitrogen

IBA,Goettingen

\subsubsection{Protein}

Bio-Rad Protein Assay

DAKO fluorescent mounting medium ECL Plus

ConcanavalinA-Sepharose 4B

Protease Inhibitor Cocktail

PD-10 Sephadex G-25 M column

Supersignal Chemiluminescence Kit

Roti-blue Colloidal Coomassie Brilliant Blue

Bovine Serum Albimin (BSA)

Iodoacetamide, Iodoacetic acid

PANSORBIN cells

Protein A sepharose

Protein $\mathrm{G}$ agarose

Prestained Marker
Bio-Rad

DakoCytomation, USA

Amersham Biosciences

Amersham Biosciences

Sigma

Amersham Pharmacia Biotech

Pierce, USA

Roth

Serva

Sigma

Calbiochem

Sigma

Sigma

Biorad

\subsubsection{Strains and Cell lines}

\subsubsection{Bacterial strains}

\begin{tabular}{|c|c|c|c|}
\hline Strain & Genotype & \multicolumn{2}{|l|}{ Reference } \\
\hline XL1-Blue & $\begin{array}{l}\text { recA1,endA1,gyrA96,th-1,hsdR17, } \\
\text { supE44,relA1,lac[F,proAB,laclqZ } \Delta M 15 \text {, } \\
\text { Tn10(Tetr)]c }\end{array}$ & Stratagene & \\
\hline BL21(DE3) & $\begin{array}{l}F^{-} \text {ompT,dcm }{ }^{+}, h s d S_{B}\left(r_{B}{ }^{-} m_{B}^{-}\right), \\
\text {Tet }^{r}, \operatorname{gal} \lambda(D E 3), \text { endA,Hte }\left[p T-T r x \mathrm{Cam}^{r}\right]\end{array}$ & (Yasukawa et al. & 1995) \\
\hline
\end{tabular}




\subsubsection{Yeast strains}

\begin{tabular}{|c|c|c|}
\hline Strain & Genotype & Reference \\
\hline AH109 & 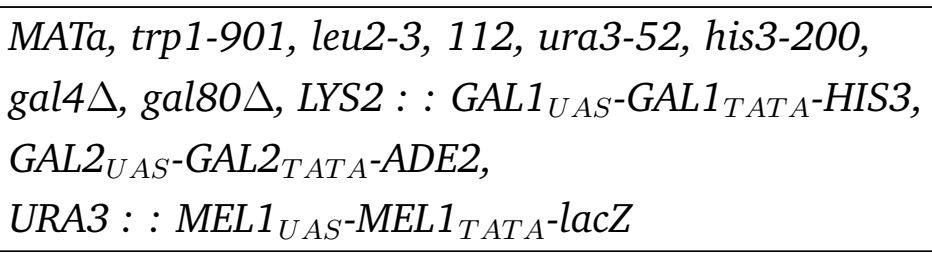 & CLONTECH \\
\hline CG1945 & $\begin{array}{l}\text { MATa, ura3-52, his3-200, ade2-101, lys2-801, } \\
\text { trp1-901, leu2-3, 112, gal4-542, gal80-538, cyh } 2 \text {, } \\
\text { LYS2 : : GAL1 } 1_{U A S}-G A L 1_{T A T A}-H I S 3 \text {, } \\
\text { URA3 : : GAL4 }{ }_{17-m e r s(x 3)}-C Y C 1_{T A T A} \text {-lacZ }\end{array}$ & CLONTECH \\
\hline
\end{tabular}

\subsubsection{Mouse cell lines}

\begin{tabular}{|c|c|c|}
\hline Cell line & Source & Reference \\
\hline E14-1 ES cells & $\begin{array}{l}\text { established from blastocysts of } \\
\text { mouse } 129 / \text { Ola strain }\end{array}$ & (Hooper et al., 1987) \\
\hline $\begin{array}{l}\text { neomycin resistant } \\
\text { MEF }\end{array}$ & $\begin{array}{l}\text { established from a } \\
\text { neomycin-resistant } \\
\text { mouse strain in this institute }\end{array}$ & (Müller et al., 1991) \\
\hline$\mu 1 \mathrm{~A}-/-\mathrm{MEF}$ & $\begin{array}{l}\text { established from the } \mu 1 \mathrm{~A}-/- \\
\text { mouse strain (mixed } \\
\text { C57 BL/6J : } 129 / \text { Ola background) }\end{array}$ & (Meyer et al., 2000) \\
\hline$\sigma 1 \mathrm{~B}-/-\mathrm{MEF}$ & $\begin{array}{l}\text { established from the } \sigma 1 \mathrm{~B}-/- \\
\text { mouse strain (mixed } \\
\text { C57 BL/6J : 129/Ola background) }\end{array}$ & $($ Riel, 2004) \\
\hline
\end{tabular}

\subsubsection{Mouse Strains}

\begin{tabular}{|l|l|l|}
\hline C57 BL/6J & Inbred strain,black colour & BRL,Switzerland \\
\hline $\mathbf{1 2 9 S V / J}$ & Inbred strain,agouti colour & BRL,Switzerland \\
\hline
\end{tabular}

\subsubsection{Vectors}

\subsubsection{Mammalian System:}

pGK Hygro Invitrogen life technologies

pUB/Bsd Invitrogen life technologies

pcDNA3.1 Invitrogen 
2.1.5.2 yeast System

$$
\begin{array}{ll}
\text { pGBT9 } & \text { BDBiosciences Clontech } \\
\text { pGBKT7 } & \text { BDBiosciences Clontech } \\
\text { pGADT7 } & \text { BDBiosciences Clontech } \\
\text { pBRIDGE } & \text { BDBiosciences Clontech }
\end{array}
$$

\subsubsection{Bacterial System}

$$
\begin{array}{ll}
\text { pGEX2T } & \text { Amersham Biosciences } \\
\text { pCR2.1 } & \text { Invitrogen }
\end{array}
$$

\subsubsection{Antibiotics and drugs}

Ampicillin

Blasticidin-S Hydrochloride

Hygromycin

Neomycin (Gentamycin sulfate or G418)

Penicillin/Streptomycin

$(100 \mathrm{x}=10,000 \mathrm{U} / \mathrm{ml})$

\section{Serva}

Invitrogen life technologies

Calbiochem

Gibco

Gibco

\subsubsection{Enzymes and Standards}

\begin{tabular}{|l|l|}
\hline Restriction endonucleases & New England Biolabs \\
\hline Klenow DNA polymerase & New England Biolabs \\
\hline DNA ligase & New England Biolabs \\
\hline Taq DNA polymerase & Amersham Pharmacia Biotech \\
\hline Alkaline phosphatase & Boehringer \\
\hline Ultrapure dNTP Set & Amersham Pharmacia Biotech \\
\hline Adenosine 5'-triphosphate (ATP) & Sigma \\
\hline 1-kb DNA ladder & Gibco BRL \\
\hline
\end{tabular}




\subsubsection{Primary antibodies}

\begin{tabular}{|c|c|c|c|c|}
\hline Antigen(epitope) & Type & WB & IF & Reference \\
\hline HA tag & mouse mAb & $1: 2000$ & $1: 100$ & Covance Inc. \\
\hline HA tag & Rat mAb & $1: 2000$ & $1: 100$ & Covance Inc. \\
\hline $\begin{array}{l}\gamma 1 \text {-adaptin } 100.3, \\
\text { (mouse } \gamma 1 \text {-adaptin hinge) }\end{array}$ & $\begin{array}{l}\text { mouse mAb, } \\
\text { purified }\end{array}$ & $1: 5000$ & $1: 100$ & $\begin{array}{l}\text { Transduction } \\
\text { Laboratories }\end{array}$ \\
\hline $\begin{array}{l}\mu 1 \mathrm{~A} \text {-adaptin } 144, \\
\text { (mouse } \mu 1 \mathrm{~A} \\
\text { residues } 295-310 \text { ) }\end{array}$ & rabbit pAb & $1: 1000$ & - & (Meyer et al., 2000) \\
\hline $\begin{array}{l}\alpha \text {-adaptin, } \\
\text { (mouse } \alpha \text {-adaptin } \\
\text { residues } 38-215 \text { ) }\end{array}$ & $\begin{array}{l}\text { mouse mAb, } \\
\text { purified }\end{array}$ & $1: 5000$ & $1: 100$ & Transduction Laboratories \\
\hline $\begin{array}{l}\text { MPR46-MSC I, } \\
\text { (cytoplasmic tail } \\
\text { of MPR46) }\end{array}$ & rabbit $\mathrm{pAb}$ & - & $1: 50$ & (Klumperman et al., 1993) \\
\hline $\begin{array}{l}\text { MPR300-I5, } \\
\text { (rat MPR300) }\end{array}$ & rabbit $\mathrm{pAb}$ & - & $1: 50$ & (Claussen et al., 1995) \\
\hline LAMP-1(1D4B) & rat $\mathrm{mAb}$ & - & $1: 100$ & $\begin{array}{l}\text { Hybridoma Bank } \\
\text { (USA) }\end{array}$ \\
\hline sortilin & mouse $\mathrm{mAb}$ & - & $1: 200$ & $\begin{array}{l}\text { C.M.Petersen } \\
\text { (Denmark) }\end{array}$ \\
\hline IL2R & mouse mAb & - & $1: 200$ & $\begin{array}{l}\text { C.M.Petersen } \\
\text { (Denmark) }\end{array}$ \\
\hline sortilin & rabbit $\mathrm{pAb}$ & $1: 200$ & - & Abcam (UK) \\
\hline
\end{tabular}

\subsubsection{Secondary antibodies}

Goat anti-rabbit Horseradish peroxidase conjugate (WB, 1:10,000)

Goat anti-mouse Horseradish peroxidase conjugate (WB,1:10,000)

Goat anti-rat Horseradish peroxidase conjugate (WB,1:10,000)

Goat anti-rabbit Alexa633 conjugate (IF,1:400)

Goat anti-mouse Alexa488 conjugate (IF,1:400)

Goat anti-rat Cy3 conjugate (IF,1:400)

All secondary antibodies were purchased from Dianova, Hamburg. 


\subsubsection{Stock solutions and buffers}

\section{M Sodiumphosphate buffer}

$1 \mathrm{M}$ solution of sodium di-hydrogen phosphate was slowly added to $1 \mathrm{M}$ di-sodium hydrogenphosphate solution with constant mixing on a magnetic stirrer till the $\mathrm{pH}$ came down to 7.4.

\section{$10 \times$ PBS}

$100 \mathrm{mM}$ sodium phosphate $\mathrm{pH} 7.4$

$9 \%$ sodium chloride

Dissolved in $800 \mathrm{ml}$ water and $\mathrm{pH}$ was adjusted to 7.4 with $\mathrm{HCl}$, volume was made up to $1000 \mathrm{ml}$ and autoclaved. Stored at room temperature.

\section{$1 \times$ TBS}

10 mM Tris/ $\mathrm{HCl}$ pH 7.4

150 mM Sodium chloride

$1 \times$ TAE 0.04 M Tris-acetate

1mM EDTA (pH 8.0)

\section{0x TAE}

$242 \mathrm{~g}$ Tris base

$57.1 \mathrm{~g}$ glacial acetic acid

$100 \mathrm{ml}$ of $0.5 \mathrm{M}$ EDTA (pH 8.0)

Dissolved in water and the final volume was made upto one litre.

\section{TE Buffer}

10 mM Tris/ HCl pH 7.5

$1 \mathrm{mM}$ EDTA

\subsection{Methods}

\subsubsection{Molecular biology}

\subsubsection{Cultivation of E.coli}

Luria Bertani (LB) medium

$10 \mathrm{~g}$ Bacto-Tryptone

$5 \mathrm{~g}$ Bacto-yeast extract

$5 \mathrm{~g} \mathrm{NaCl}$

Dissolved in $900 \mathrm{ml}$ distilled water, $\mathrm{pH}$ adjusted to 7.0 with $10 \mathrm{~N} \mathrm{NaOH}$, made up the volume to one liter, sterilized by autoclaving and stored at room temperature. LB-Ampicillin Agar Plates 
1.5\% of Agar was added to the LB medium and autoclaved. After autoclaving, the medium was let to cool down to $55{ }^{\circ} \mathrm{C}$ and ampicillin was added to a final concentration of $100 \mu \mathrm{g} / \mathrm{ml}$. This medium was poured into $10 \mathrm{~cm}$ petriplates in the hood and left undisturbed for about $30 \mathrm{~min}$ to solidify. LB-Agar plates were stored in the cold room.

\subsubsection{Preparation of electrocompetent Bacterial cells}

10\% (v/v) Glycerol : $1 \mathrm{ml}$ (1.26 g) of glycerol in $10 \mathrm{ml}$ sterile water

A single E. coli colony was inoculated into $10 \mathrm{ml}$ of LB media and allowed to grow overnight at $37{ }^{\circ} \mathrm{C}, 250 \mathrm{rpm}$ in a shaker incubator. This pre-culture was diluted 1:100 into fresh LB media and allowed to grow to an $\mathrm{OD}_{600}$ of $0.3-0.35$ at $37{ }^{\circ} \mathrm{C}$. The culture was pre-chilled on ice for $15 \mathrm{~min}$ and centrifuged at $5000 \mathrm{rpm}$ for 15 min at $4{ }^{\circ} \mathrm{C}$ in a JA-10 rotor. The pellet was resuspended in $500 \mathrm{ml}$ of ice cold water and centrifuged as described above. This washing was repeated once again with $250 \mathrm{ml}$ ice cold water. The resulting pellet was resuspended in $20 \mathrm{ml}$ of filter sterile ice-cold $10 \%$ glycerol and centrifuged at $4{ }^{\circ} \mathrm{C}$ for $15 \mathrm{~min}$ in a JA-20 rotor.The pellet was resuspended in $2 \mathrm{ml}$ of ice-cold $10 \%$ glycerol. Aliquots of $40 \mu \mathrm{l}$ were quick frozen in dry-ice and stored at $-80{ }^{\circ} \mathrm{C}$.

\subsubsection{Transformation of plasmid DNA by Electroporation}

SOC medium

$0.5 \%$ Yeast extract

$2 \%$ Bacto-tryptone

$10 \mathrm{mM}$ Sodium chloride

2,5 mM Potassium chloride

$10 \mathrm{mM}$ Magnesium sulfate

$10 \mathrm{mM}$ Magnesium chloride

$20 \mathrm{mM}$ Glucose

For each electroporation, a $40 \mu \mathrm{l}$ aliquot of the electrocompetent cells was thawed on ice and 1-5 $\mu$ l of the DNA sample was added and mixed.The resulting mixture was transferred into a sterile, pre-chilled electroporation cuvette and subjected to a $1800 \mathrm{~V}$ pulse in the electroporator. Immediately after the pulse, the cells were diluted in $500 \mu \mathrm{l}$ of ice-cold SOC medium and transferred to a sterile e-cup and incubated at $37{ }^{\circ} \mathrm{C}$ with mild shaking for 30-40 min. After the recovery period, the cells were plated onto LB plates supplemented with the appropriate antibiotic and allowed to grow over night at $37^{\circ} \mathrm{C}$. 


\subsubsection{Preparation of Glycerol stocks for bacterial strains}

Bacterial cultures were grown to $\log$ phase at $37{ }^{\circ} \mathrm{C}, 250 \mathrm{rpm}$ in a shaker-incubator. $0.7 \mathrm{ml}$ of this logarithmic culture was mixed with $0.3 \mathrm{ml}$ of sterile $100 \%$ glycerol. The contents were gently mixed, shock frozen on dry ice and stored at $-80{ }^{\circ} \mathrm{C}$.

\subsubsection{Plasmid DNA preparation and cloning protocols}

All required instructions from the manufacturer were followed for the isolation of plasmid DNA (Qiaprep Kit), for the digestion or modificaion of DNA by enzymes, for the analysis of DNA by agarose gel electrophoresis in TAE buffer and for the purification of DNA fragments from sliced agarose gels (Qiaspin Gel Extraction Kit).

\subsubsection{Determining the concentration of DNA}

DNA concentration was determined using a spectrophotometer. DNA was diluted in water and the absorbance was measured at $260 \mathrm{~nm}$. Absorbance or optical density (OD) of 1 at $260 \mathrm{~nm}$ corresponds to $\sim 50 \mu \mathrm{g} / \mathrm{ml}$ of double stranded DNA or $\sim 40 \mu \mathrm{g} / \mathrm{ml}$ of single stranded DNA and RNA or $\sim 20 \mu \mathrm{g} / \mathrm{ml}$ of oligonucleotides. The ratio between the readings at $260 \mathrm{~nm}$ and $280 \mathrm{~nm}$ (OD260/ OD280) provides an estimate of the purity of the nucleic acid. Pure preparations of DNA and RNA have OD-260/OD-280 values of 1.8 and 2.0, respectively. Any contamination with proteins or phenol would yield values less than mentioned above.

\subsubsection{Restriction endonuclease digestion of DNA}

The activity of restriction enzymes is measured in terms of 'Units' (U).

One unit of restriction enzyme is the amount of enzyme required to completely digest $1 \mu \mathrm{g}$ substrate DNA in $1 \mathrm{hr}$.

$\begin{array}{ll}\text { Plasmid DNA } & 0.5-1 \mu \mathrm{g} \\ 10 \mathrm{x} \text { buffer } & 2 \mu \mathrm{l} \\ \text { Restriction enzyme } & 1-2 \mathrm{U} \\ \text { BSA } & 1 \mu \mathrm{g} / \mu \mathrm{l} \text { (added where essential) }\end{array}$

Water was added to a final volume of $20 \mu \mathrm{l}$. Reaction mix was incubated at 37 ${ }^{o} \mathrm{C}^{*}$ for 2 hours.

*Incubation temperatures were set as recommended by the manufacturer (New England Bio Labs Beverly U.S.A.) which varies from enzyme to enzyme. Reaction mix was analyzed on agarose gel $(0.8$ to $2 \%$ depending on the size of the DNA of 
interest) The buffers and enzymes used were all from New England Biolabs. The composition of the buffers is as follows:

NEB 1: $10 \mathrm{mM}$ Bis Tris Propane-HCl (pH 7.0), $10 \mathrm{mM} \mathrm{MgCl}_{2}, 1 \mathrm{mM}$ DTT

NEB 2: $10 \mathrm{mM}$ Tris-HCl (pH 7.9), $10 \mathrm{mM} \mathrm{MgCl}_{2}, 50 \mathrm{mM} \mathrm{NaCl}, 1 \mathrm{mM}$ DTT

NEB 3: 50 mM Tris-HCl (pH 7.9), $10 \mathrm{mM} \mathrm{MgCl}_{2}, 100 \mathrm{mM} \mathrm{NaCl}, 1 \mathrm{mM}$ DTT

NEB 4: 20 mM Tris-acetate (pH 7.9), 10 mM Magnesium acetate, 1 mM DTT, 50 $\mathrm{mM}$ Potassium acetate

\subsubsection{Agarose gel electrophoresis of DNA}

The size and purity of DNA is analyzed by agarose gel electrophoresis. Concentration of agarose used for analysis is inversely proportional to the size of the DNA of interest, that is, the larger the DNA the lower the concentration of agarose.

\begin{tabular}{|c|c|}
\hline Agarose concentration (\%) & DNA size $(\mathrm{kb})$ \\
\hline 0.7 & $20-1$ \\
0.9 & $7-0.5$ \\
1.2 & $6-0.4$ \\
1.5 & $4-0.2$ \\
2.0 & $3-0.1$ \\
\hline
\end{tabular}

Gel loading buffer (10x)

$0.25 \%(\mathrm{w} / \mathrm{v})$ Bromophenol blue

$40 \%$ Saccharose in $1 \mathrm{x}$ TAE

Agarose was weighed and dissolved in $1 \mathrm{x}$ TAE by boiling in microwave oven. The agarose solution was cooled to $60{ }^{\circ} \mathrm{C}$ and ethidium bromide was added to a final concentration of $0.5 \mu \mathrm{g} / \mathrm{ml}$. This was poured into the agarose gel cassette and allowed to polymerize completely. The sample DNA was mixed with gel loading buffer and loaded onto the gel. The gel electrophoresis was carried out at $100 \mathrm{~V}$. Ethidium bromide is a fluorescent dye which contains a planar group that intercalates between the stacked bases of the DNA. The fixed position of this group and its close proximity to the bases cause the dye to bind DNA. It results in an increased fluorescent yield compared to that of the dye in free solution. Ultraviolet radiation at $254 \mathrm{~nm}$ is absorbed by the DNA and transmitted to the dye; radiation at $302 \mathrm{~nm}$ and $366 \mathrm{~nm}$ is absorbed by the bound dye itself. In both cases, the energy is re-emitted at $590 \mathrm{~nm}$ in the red orange region of the visible spectrum. Hence DNA can be visualized under a UV transilluminator. The gel can be photographed using a gel documentation system. 


\subsubsection{Isolation of RNA from mouse cells or mouse tissue}

All the preparations were done according to the RNeasy protocol (Qiagen) with the following details: For isolation of total RNA from cells, they were grown to confluency on a $10 \mathrm{~cm}$ culture dish and lysed directly on the plate. The lysate was eventually stored at $-80^{\circ} \mathrm{C}$. For isolation of total RNA from tissue, the organs were excised from a mouse and immediately either frozen in liquid nitrogen for storage at $-80{ }^{\circ} \mathrm{C}$ or immersed in RNAlater reagent $(10 \mathrm{ml} / \mathrm{g}$ tissue $)$ for storage at $4{ }^{\circ} \mathrm{C}$ or at lower temperature. An appropriate amount of tissue was cut into mm-sized pieces and homogenised with an ultra-turrax. For tissues with a low yield of RNA such as brain, heart etc., about twice the recommended tissue weight could be successfully loaded onto one column, if the volume of lysis buffer and subsequent buffers was doubled as well.

\subsubsection{Polymerase chain reaction (PCR)}

PCR is a rapid and versatile in vitro method for amplifying defined target DNA fragments by DNA polymerase which occurs naturally in living organisms, where it duplicates DNA when cells divide. It is used to amplify a short, well-defined part of a DNA strand. Taq polymerase is widely used but the disadvantage is that it lacks $3 \rightarrow 5$ ' proof reading exonuclease activity, leading to mutations in the sequence. For amplification of DNA fragments of size above $1 \mathrm{~kb}$, Expand high fidelity Taq polymerase (Roche) was used.

\begin{tabular}{lll}
\hline Condition & Temperature & Time \\
\hline Denaturation & $95^{\circ} \mathrm{C}$ & $2 \mathrm{~min}$ \\
Amplification & $95^{\circ} \mathrm{C}$ & $40 \mathrm{sec}$ \\
& $55^{\circ} \mathrm{C}$ & $40 \mathrm{sec}$ \\
& $72^{\circ} \mathrm{C}$ & $1 \mathrm{~min}$ for upto $1.5-2 \mathrm{~kb}$ \\
Extention & $72^{\circ} \mathrm{C}$ & $10 \mathrm{~min}$ \\
End & $4{ }^{\circ} \mathrm{C}$ & $\infty$ \\
\hline
\end{tabular}




\title{
PCR Mix
}

\begin{tabular}{lr}
\hline Components & Volume \\
\hline 10X Taq buffer (100 mM Tris/HCl pH 9.0; 500 mM KCl) & $10 \mu \mathrm{l}$ \\
$2.5 \mathrm{mM}$ dNTPs (Amersham, Stock 100 mM) & $10 \mu \mathrm{l}$ \\
$25 \mathrm{mM} \mathrm{MgCl}_{2}$ & $5 \mu \mathrm{l}$ \\
5-primer (Stock $100 \mathrm{pmol} / \mu \mathrm{l})$ & $2 \mu \mathrm{l}$ \\
3-primer (Stock 100 pmol/ $\mu \mathrm{l})$ & $2 \mu \mathrm{l}$ \\
Template DNA (40 ng/ $\mu \mathrm{l})$ & $1 \mu \mathrm{l}$ \\
Taq polymerase $+1 / 10$ Pfu-Polymerase & $1 \mu \mathrm{l}$ \\
Final volume made upto $100 \mu$ l with HPLC water & \\
\hline
\end{tabular}

\subsubsection{Ligation}

Joining linear DNA fragments together by a phosphodiester bond between the 3' hydroxyl of one nucleotide and the 5' phosphate of another is called ligation. T4 DNA ligase is used for this purpose, it ligates the cohesive ends of the fragments. There are two types of ligation viz., cohesive end or sticky end ligation and blunt end ligation, depending on the restriction digestion of the DNA. For the blunt end ligation and also if digestion was with single sticky end enzyme, the vector should be dephosphorylated by alkaline phosphatase, and the ligation mix should be incubated for overnight.

For example:

\author{
$\mathrm{x} \mu \mathrm{l} \quad$ linearised vector DNA \\ y $\mu \mathrm{l}$ Insert DNA [1(vector DNA):10 (insert DNA)] \\ $1.5 \mu \mathrm{l} \quad$ 10x Ligase buffer \\ $1.0 \mu \mathrm{l}$ T4-DNA-Ligase $(400 \mathrm{U} / \mu \mathrm{l})$
}

Made upto $10 \mu \mathrm{l}$ with $\mathrm{ddH}_{2} \mathrm{O}$, incubated overnight at $16^{\circ} \mathrm{C}$ or $1 \mathrm{hr}$ at RT and transformed into E.coli competent cells.

\subsubsection{Sequencing PCR}

DNA sequencing was done by Sanger's method using a DNA sequencer. The plasmid DNA to be sequenced was supplied with a mixture of all four nucleotides plus four ddNTPs, each labeled with a tag that fluoresces different color. For sequencing reaction, clean DNA should be used (prepared by QIAprep-Spin-Miniprep-Kit). 
For example:

$\mathrm{x} \mu \mathrm{l} \quad$ Plasmid-DNA $(0.2-0.5 \mu \mathrm{g})$

$3.2 \mu \mathrm{l} \quad$ Forward primer or reverse primer $(1 \mathrm{pmol} / \mu \mathrm{l})$

$2.0 \mu \mathrm{l}$ ABI PRISM Dye Terminator Cycle Sequencing Ready Reaction Premix

$\mathrm{x} \mu \mathrm{l} \quad$ HPLC- $\mathrm{H}_{2} \mathrm{O}$ (Make it upto $10 \mu \mathrm{l}$ )

\begin{tabular}{lll}
\hline Condition & Temperature & Time \\
\hline Denaturation & $96{ }^{\circ} \mathrm{C}$ & $10 \mathrm{sec}$ \\
Amplification & $96{ }^{\circ} \mathrm{C}$ & $10 \mathrm{sec}$ \\
& $50^{\circ} \mathrm{C}$ & $5 \mathrm{sec}$ \\
& $60^{\circ} \mathrm{C}$ & $4 \mathrm{~min}$ \\
25 cycles & & \\
End & $4{ }^{\circ} \mathrm{C}$ & $\infty$ \\
\hline
\end{tabular}

The PCR product for sequencing should be cleaned thoroughly to remove fluorescently labeled free ddNTPs to avoid background. To the PCR product $2 \mu \mathrm{l}$ of $3 \mathrm{M}$ Sodium acetate ( $\mathrm{pH}$ 5.2) and $50 \mu \mathrm{l} 95 \%$ ethanol (room temperature) was added, incubated at RT for $10 \mathrm{~min}$ and centrifuged for $20 \mathrm{~min}$ at $13000 \mathrm{rpm}$. The pellet was washed with $250 \mu \mathrm{l}$ of $70 \%$ ethanol for $10 \mathrm{~min}$ at $13000 \mathrm{rpm}$, dried and resuspended in $25 \mu \mathrm{l}$ of HPLC water and given for sequencing.

\subsubsection{Yeast Genetics}

\subsubsection{Lithium acetate mediated yeast transformation}

\section{Stock solutions}

50\% PEG (3350) Sterilize with $0.22 \mu \mathrm{m}$ cellulose-acetate membrane filter

10X LiAc (1M ) Adjust $\mathrm{pH}$ to 7.5 with Glacial acetic acid.

Filter sterilize as above

10X TE

$100 \mathrm{mM}$ Tris- $\mathrm{HCl} \mathrm{pH} 8.0$

10 mM EDTA pH 8.0

Sterilize by autoclaving

This method is extremely efficient to transform PCR products, plasmids and libraries. The yeast cells are made competent freshly before the transformation. All materials used for this procedure should be sterilized well. Preparation of competent cells. 
Inoculate a single colony of yeast into $5 \mathrm{ml}$ YAPD medium and grow overnight. Inoculate $250 \mathrm{ml}$ of YAPD medium with $5 \mathrm{ml}$ of pre-inoculum and grow for 4- 5 hr (OD600 $0.6-0.8$ ) at $30{ }^{\circ} \mathrm{C}$ and $225 \mathrm{rpm}$. Pellet the cells at 5,000 rpm for 15 min at $4{ }^{\circ} \mathrm{C}$ in JA-20 rotor. Washed the pellet twice with ice cold sterile water. Resuspended the pellet in $3 \mathrm{ml} 1 \mathrm{X}$ LiAc/TE sterile.

Incubate yeast competent cells for about $30 \mathrm{~min}$ at $30^{\circ} \mathrm{C}$ and aliquot $100 \mu \mathrm{l}$ for transformation by making a cocktail as follows.

\begin{tabular}{lr}
\hline Ingredients & Volume or Amount \\
\hline Yeast cells in LiAc/TE & $100 \mu \mathrm{l}$ \\
PEG/LiAc (sterile) & $700 \mu \mathrm{l}$ \\
SSDNA (Salmon/Herring sperm DNA) & $5 \mu \mathrm{l}$ \\
pDNA (in less than $5 \mu \mathrm{l})$ & $0.1 \mu \mathrm{g}$ \\
\hline
\end{tabular}

Incubate the cells at $30{ }^{\circ} \mathrm{C}$ for 30 to $60 \mathrm{~min}$. After the incubation time, the mixture heat shock to the cells at $42{ }^{\circ} \mathrm{C}$ for $15 \mathrm{~min}$. Spin the cells for $1 \mathrm{~min}$ at 2000 $\mathrm{rpm}, 4^{\circ} \mathrm{C}$ in a tabletop centrifuge. Discard the supernatant. Wash the cells at least once with $1 \mathrm{ml}$ of water at room temperature so that PEG is completely washed out (PEG can induce fusion of cells). Resuspend the pellet in $200 \mu \mathrm{l}$ of sterile water and plate on appropriate plate lacking the auxotrophic marker. Incubate the cells at 30 ${ }^{\circ} \mathrm{C}$ for 3 to 5 days until colonies appear.

\subsubsection{Yeast cell plasmid rescue}

\section{Reagents}

Appropriate SC medium

TE $\mathrm{pH}-7.5$

$3 \%$ SDS

$0.2 \mathrm{~N} \mathrm{NaOH}$

$3 \mathrm{M} \mathrm{CH} \mathrm{COONa}_{3}$

$\mathrm{pH}-5.3$

Phenol Chloroform Absolute alcohol and 70\% ethanol

Yeast cells with the pDNA were grown $(\sim 24-32 \mathrm{hr})$ in selective medium. Pelleted the cells $1.5 \mathrm{ml}$ of well grown cell culture of yeast and resuspended in $100 \mu \mathrm{l}$ of lysis buffer (TE $\mathrm{pH}-7.5,3 \%$ SDS and $0.2 \mathrm{~N} \mathrm{NaOH}$ ) and incubated at room temperature for $30 \mathrm{~min}$. To the above lysate $600 \mu \mathrm{l}$ of TE $\mathrm{pH}-7.5$ was added, followed by the 
addition of $70 \mu \mathrm{l}$ of $3 \mathrm{M}$ sodium acetate $\mathrm{pH}-5.3$. Vortex and extract sequentially with phenol/chloroform $(1: 1)$ and chloroform. Precipitated the plasmid pDNA in the aqueous supernatant with two volumes of ethanol. Incubate at $-20{ }^{\circ} \mathrm{C}$ for an hour. Pelleted the pDNA at $12,000 \mathrm{rpm}$ for $10 \mathrm{~min}$ at $4{ }^{\circ} \mathrm{C}$. Rinse the pellet with $70 \%$ ethanol and resuspended the pDNA in $20 \mu \mathrm{l}$ of TE or sterile water. $2 \mu \mathrm{l}$ of this is used to transform in E.coli cells by electroporation. The transformants were selected on LB plates containing appropriate antibiotic marker.

\subsubsection{Testing interaction by growth}

The growth patterns of different yeast transformants were compared on selection plates by growth test either by dropping nearly equal number of cells or by streaking. For drop out the transformant were inoculated in $8 \mathrm{ml}$ of YEPD or $\mathrm{SD}$-medium and grown overnight at $24^{\circ} \mathrm{C}$ till $\mathrm{OD}_{600} 0.2-1.0$. The selection plates SD/-Leu/-Trp, SD/-His/-Leu/-Trp, SD/-Ade/-Leu/-Trp and SD/-Ade/-His/-Leu/-Trp plates were pre-warmed at $30^{\circ} \mathrm{C}$. For each strain, two dilutions were prepared in sterile water viz., $0.01 \mathrm{OD} / \mathrm{ml}$ and $0.05 \mathrm{OD} / \mathrm{ml} .10 \mu \mathrm{l}$ from each dilution is spotted onto the pre-warmed plates using a pattern. The spots were allowed to dry and the plates were incubated at $30{ }^{\circ} \mathrm{C}$. The growth was scored after $24 \mathrm{hr}$ and the strength of interaction was assessed based on the growth of yeast; faster growth implicates strong interaction.

Other approach for growth test was by streaking the transormants on the selection plates SD/-Leu/-Trp, SD/-His/-Leu/-Trp, SD/-Ade/-Leu/-Trp and SD/Ade/-His/-Leu/-Trp plates were pre-warmed at $30{ }^{\circ} \mathrm{C}$. Monitored the growth after 36-48 hrs.

\subsubsection{Filter lift assay - lacZ reporter gene}

\section{Reagents}

\begin{tabular}{ll}
\hline Components & Amount (g) \\
\hline $\mathrm{NaH}_{2} \mathrm{PO}_{4} \cdot \mathrm{H}_{2} \mathrm{O}$ & 13.79 \\
$\mathrm{KCl}$ & 0.75 \\
$\mathrm{MgSO}_{4} \cdot 7 \mathrm{H}_{2} \mathrm{O}$ & 0.246 \\
\hline
\end{tabular}

X-Gal(5-bromo-4-chloro-3-indolyl- $\beta$-d-galactopyranoside):(20 mg/ml) Disslove one gram of X-Gal in $50 \mathrm{ml}$ of $\mathrm{N}, \mathrm{N}$-dimethylformamide. Store at $-20^{\circ} \mathrm{C}$. 


\section{$\mathrm{Z}$ buffer/ $\beta$-mercaptoethanol}

This should be made fresh by adding $270 \mu \mathrm{l}$ of -mercaptoethanol to $100 \mathrm{ml}$ of $\mathrm{Z}$ buffer.

\section{Z buffer/ $\beta$-mercaptoethanol/X-Gal}

This should be made fresh by adding $270 \mu \mathrm{l}$ of $\beta$-mercaptoethanol and $1.67 \mathrm{ml}$ of $\mathrm{X}$-Gal solution $(20 \mathrm{mg} / \mathrm{ml})$ to $100 \mathrm{ml}$ of $\mathrm{Z}$ buffer.

Yeast transformants to be checked for the $\beta$-galactosidase (lac $Z$ reporter gene) were streaked on SD/-Leu/-Trp plates and grown for 36-48 hr. Carefully placed a sterile $75 \mathrm{~mm}$ circle of Whatmann No.1 filter paper on top of the colonies or patches growing on selective medium. Ensured that filter paper makes good contact with the colonies. Mark the orientation of the filter paper relative to the plate using an 18-guage needle to punch through the filter in an asymmetric pattern. Remove the filter paper from the plate with a clean forceps after it has become fully absorbed to the colonies and immerse in liquid nitrogen for 10-15 sec. Filter paper was removed from the the liquid nitrogen and thawed by placing on a piece of plastic wrap with colony side up. Repeat freeze and thaw cycle twice. Placed another $75 \mathrm{~mm}$ sterile Whatman No. 1 filter into an empty petri plate and dispense $1.25 \mathrm{ml}$ of $\mathrm{Z}$ buffer/ $\beta$ mercaptoethanol/X-Gal onto the filter, on top of this placed the filter with colony side up. Wrap it with aluminium foil and incubate at $37^{\circ} \mathrm{C}$ for $24 \mathrm{hr}$.

\subsubsection{X- $\alpha$-Gal assay}

Mel1 is an endogenous gene found in several yeast strains because $\alpha$-galactosidase is a secreted enzyme, it can be assayed directly on X- $\alpha$-Gal (No.8061-1, BD Bioscience) indicator plates, which employs blue/white screening. Yeast transformants to be checked for the $\alpha$-galactosidase (Mel1 reporter gene) were streaked on SD/-Leu/Trp or SD/-Ade/-His/-Leu/-Trp plates containing X- $\alpha$-Gal and grown for 36-48 hr and monitored for the color development. 


\subsubsection{Cell culture and transfections}

\subsubsection{Basics}

Normal growth medium:

Trypsin-EDTA solution:

phosphate-buffered saline (PBS):

Freezing medium:
DMEM, 10\% fetal calf serum, Pen-Strep

$0.5 \mathrm{~g} / 1$ Trypsin, $0.2 \mathrm{~g} / 1$ EDTA

$150 \mathrm{mM} \mathrm{NaCl}, 120 \mathrm{mM} \mathrm{KCl}$, $10 \mathrm{mM} \mathrm{Na}{ }_{2} \mathrm{HPO}_{2} / \mathrm{KH}_{2} \mathrm{PO}_{2} \mathrm{pH} 7.4$

$10 \%$ DMSO in normal growth medium

All cell lines were grown in Dulbecco's modified Eagle's medium (DMEM) supplemented with $10 \%$ fetal calf serum (FCS) and 1\% penicillin/streptomycin (Invitrogen) under $5 \% \mathrm{CO}_{2}$ at $37^{\circ} \mathrm{C}$.

\subsubsection{Passaging by trypsinisation}

Cell line was passaged every 2-7 days depending on the growth rate. After two washes with PBS, $0.5 \mathrm{ml}$ trypsin solution per $25 \mathrm{~cm}^{2}$ was added to the cell layer. The cells were incubated at $37^{\circ} \mathrm{C}$ for $5 \mathrm{~min}$, then singled by tapping to the dish and resuspended in medium. To concentrate, wash or harvest cells, centrifugation was always done at 500xg for $5 \mathrm{~min}$.

\subsubsection{Freezing cells for stock maintenance}

Usually, cells from a confluent $35 \mathrm{~cm}^{2}$ flask were frozen as 2-4 vials. Cells were pelleted after trypsinisation, resuspended in $1 \mathrm{ml}$ freezing medium per vial, immediately placed on ice and stored at $-80{ }^{\circ} \mathrm{C}$ overnight. The vials were transferred to the liquid nitrogen container the following day.

\subsubsection{Thawing cells}

The frozen vial was taken out of liquid nitrogen, the cap was carefully loosened to release pressure and the vial was thawed in $70 \%$ ethanol at $37{ }^{\circ} \mathrm{C}$, until the suspension had almost completely melted. Under the hood, the cells were then transferred to a tube containing $5 \mathrm{ml}$ chilled cell culture medium. The cells were pelleted, resuspended in prewarmed culture medium and transferred to a culture flask. Medium was replaced on the next day. 


\subsubsection{Transient transfection}

Transient transfection was performed with Lipofectamine 2000 (Invitrogen) or magnetic bead assisted transfection(MaTra)(IBA, Goettingen) and followed the recommended protocol. Typically, $1 \mu \mathrm{g}$ of plasmid DNA/well were used for transfection in a 24 well plate. Approximately 18 hours after transfection, the cells were processed for subsequent analysis.

\subsubsection{Biochemical Methods}

\subsubsection{Protein estimation by BIORAD reagent}

Bovine Serum Albumin (BSA) stock solution $1 \mathrm{mg} / \mathrm{ml}$

Concentration range : $2-16 \mu \mathrm{g} / \mu \mathrm{l}$

A standard curve was made using BSA in the range of $2-16 \mu \mathrm{g} .10 \mu \mathrm{l}$ of the sample was used for the protein estimation. The sample volume was made up to $800 \mu \mathrm{l}$ with water. $200 \mu \mathrm{l}$ of the BIORAD reagent was added to each samples, votexed and incubated for 3 - $5 \mathrm{~min}$ at room temperature, $200 \mu \mathrm{l}$ from each tube was pipetted into ELISA strips and optical density was measured at $595 \mathrm{~nm}$ in the ELISA reader.

\subsubsection{Solubilization of proteins}

$2 \times$ Laemmli buffer

125 mM Tris-Cl, pH 6.8

$4 \%$ SDS

0.004\%Bromophenol blue

$20 \%(\mathrm{w} / \mathrm{v})$ Glycerol

$10 \% \beta$-mercaptoethanol

Protein samples dried by speed vac were resuspended in $1 \mathrm{x}$ Laemmli buffer. The resuspended proteins were boiled at $95{ }^{\circ} \mathrm{C}$ for $5 \mathrm{~min}$, cooled on ice for $1 \mathrm{~min}$, centrifuged at 13,000 rpm for $2 \mathrm{~min}$ and resolved by SDS-PAGE.

\subsubsection{SDS-Polyacrylamide Gel Electrophoresis}

The elecrophoretic separation of the proteins was performed through high-tris discontinuous SDS polyacrylamide gel electrophoresis. A system with vertical oriented glass plates ( $16 \times 16 \mathrm{~cm} ; 1 \mathrm{~mm}$ spacer) was used. The gels were prepared as follows: first the resolving gel (12.5-17.5\% acrylamide) was put between glass plates and covered with a layer of butanol. After polymerisation of the gel was completed, butanol was removed and the space between glass plates was dried with Whatmann 
paper. Then the concentrating gel was put on top of the polymerised resolving gel and the sample combs were introduced immediately. After polymerization was complete, the combs were removed. The glass plates with the gel between them were fixed inside the electrophoresis chamber and covered with electrophoresis buffer. Protein samples were mixed with Laemmli loading buffer 1:1, denatured by $95{ }^{\circ} \mathrm{C}$ and centrifuged at $14000 \mathrm{rpm}$ in a table-top centrifuge at room temperature. The supernatant was introduced into the stacking gel pockets. Electrophoresis was performed for 2-3 hours with the constant current of $35 \mathrm{~mA}$.

Electrophoresis buffer:

SDS (w/v) - 10g/10 litre

Glycine (w/v) - 144,27g/10 litre

Tris (w/v) - 60,53g/10 litre

20\% Ammoniumperoxiddisulfate (APS) in ddH2O

\subsubsection{Stacking gel(1X)}

\begin{tabular}{ll}
\hline Ingredients & $5 \%$ \\
\hline 30\% Acrylamide solution: (ml) & 0.4 \\
1\% bisacrylamide solution (ml) & 0.275 \\
ddH2O (ml) & 1.175 \\
0,5 M Tris- $\mathrm{HCl}, \mathrm{pH} 6.8(\mathrm{ml})$ & 0.625 \\
$(10 \%)$ SDS $(\mu \mathrm{l})$ & 25 \\
TEMED $(\mu \mathrm{l})$ & 2.5 \\
$(20 \%)$ APS $(\mu \mathrm{l})$ & 10 \\
\hline
\end{tabular}

\subsubsection{Separating gel (1X)}

\begin{tabular}{ll}
\hline Ingredients & $15 \%$ \\
\hline 30\% Acrylamide solution: $(\mathrm{ml})$ & 8.75 \\
1\% bisacrylamide solution $(\mathrm{ml})$ & 2.74 \\
ddH2O (ml) & 1.4 \\
$1.5 \mathrm{M}$ Tris- $\mathrm{HCl}, \mathrm{pH} 8.8(\mathrm{ml})$ & 4.3 \\
$(10 \%)$ SDS $(\mu \mathrm{l})$ & 175 \\
TEMED $(\mu \mathrm{l})$ & 14.58 \\
$(20 \%)$ APS $(\mu \mathrm{l})$ & 58.33 \\
\hline
\end{tabular}




\subsubsection{Staining with Roti-Blue Colloidal Coomassie}

Colloidal Coomassie staining is one of the most sensitive staining protocols. Due to the colloidal properties of the dye, it binds with high specificity to proteins and only minimal to the gel matrix. This allows visualisation of proteins separated by SDS-PAGE with sensitivity as high as $30 \mathrm{ng}$ of protein. Roti-Blue collodial Coomassie was purchased from Roth and staining was performed according to the protocol as follows. Immediately after completion of electrophoresis, gels were incubated in the fixing solution for $60 \mathrm{~min}$, shaking. The staining solution was applied on to the gels for $60 \mathrm{~min}$. In special cases the gels were stained for several days, which improved the sensitivity of the staining. After staining, gels were incubated in the washing solution for 5-10 min and then kept in the stabilising solution or prepared for drying in the drying solution.

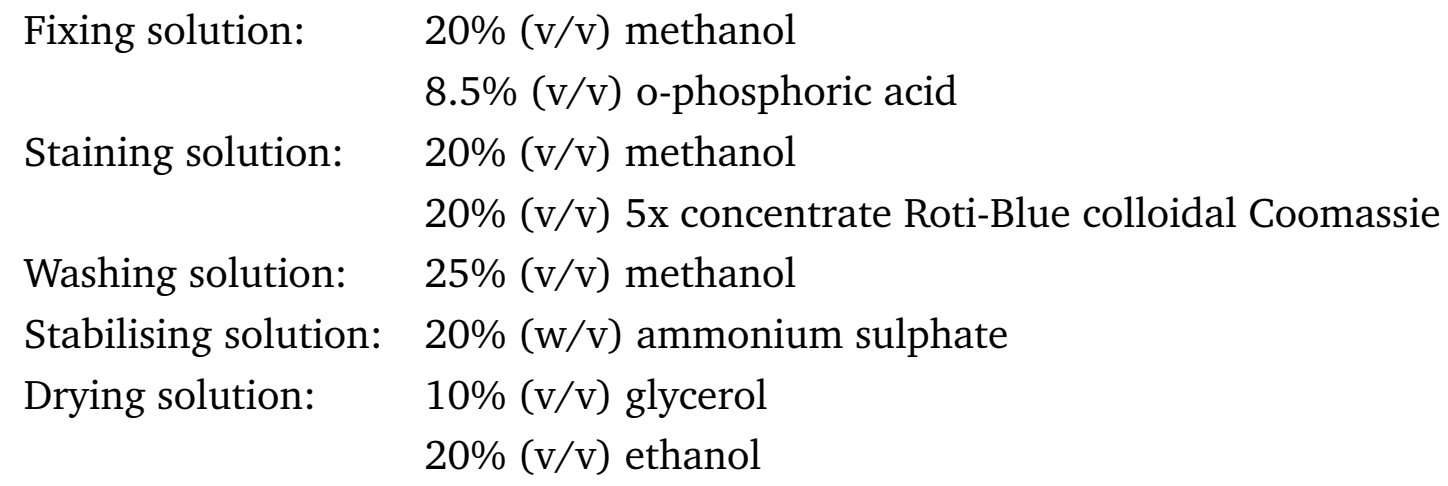

\subsubsection{Western blot (semi-dry)}

Cathode buffer

Anode buffer

Phosphate-buffered saline (PBS)

TBST

Blocking buffer

stripping solution

SDS stripping solution
$40 \mathrm{mM}$-aminocaproic acid, $20 \mathrm{mM}$ Tris-Cl, 20\% (v/v) methanol, pH 9.0 (pH was adjusted with free Tris base before addition of methanol) 75 mM Tris-Cl, 20\% (v/v) methanol, pH 7.4 $140 \mathrm{mM} \mathrm{NaCl}, 2.7 \mathrm{mM} \mathrm{KCl}, 10 \mathrm{mM}-$ $\mathrm{Na}_{2} \mathrm{HPO} 4,1.8 \mathrm{mM} \mathrm{KH} \mathrm{PO}_{2}$, $\mathrm{pH} 7.3$ $0.1 \%(\mathrm{v} / \mathrm{v})$ Tween-20 in TBS $5 \%$ milk powder in TBST $0.2 \mathrm{M} \mathrm{NaOH}$ 16 mM Tris-Cl pH 6.8, 2\% SDS, $0.1 \mathrm{M}$-mercaptoethanol

Six pieces of $3 \mathrm{~mm}$ Whatman paper and one piece of nitrocellulose membrane were cut to the size of the SDS gel. Gel and membrane were equilibrated for 5-15 min 
in cathode buffer. The blot was assembled without air bubbles according to the following scheme:

cathode (-)

3x paper in cathode buffer

gel

membrane

3x paper in anode buffer

anode $(+)$

For transfer, the current was set to $1 \mathrm{~mA} / \mathrm{cm}^{2}$ gel size for $45-60 \mathrm{~min}$. The membrane was then briefly washed with PBST and incubated in blocking buffer for one hour at room temperature. Decoration with the primary antibody diluted in blocking buffer was performed by incubating overnight at $4^{\circ} \mathrm{C}$. After three 30-45 min washes with TBST, the membrane was incubated with horse radish peroxidase (HRP)-coupled secondary antibody, diluted 1:10,000 in blocking buffer, for one hour at room temperature. The blot was washed three times 30-45 min with PBST and incubated with chemiluminescence substrate solution. Intelligent Dark Box II Camera was used for signal detection.

\subsubsection{Stripping of nitrocellulose membranes}

Removal of antibodies from a blot was done under mild conditions, if it should serve to reduce the background for incubation with another primary antibody, either from a different species or for a protein of clearly distinct size than in the first decoration. After washing the membrane in TBST, it was incubated 5-10 min in 0.2 $\mathrm{M} \mathrm{NaOH}$ stripping solution. Washed the membrane in distilled water for $10 \mathrm{~min}$. For stringent stripping, the blot was incubated in SDS stripping solution for $30 \mathrm{~min}$, tightly closed, on a wheel at $50{ }^{\circ} \mathrm{C}$, followed by several washes in TBST. Decoration of the membrane was done as described above, starting from the blocking step again. 


\subsubsection{Preparation of cytosol fraction for pull-down assay}

Buffer-A

PBS containing protease inhibitors

1 mM EDTA

$1 \mathrm{mM}$ PMSF

5 mM IAA (iodo-acetamide)

$0.1 \%$ Triton X-100

Cells were grown to confluency in a $10 \mathrm{~cm}$ dish till near-confluency in DMEM medium. They were washed twice with cold PBS and scraped in lysis buffer A, sonicated $3 \mathrm{x} \mathrm{10s}$ and centrifuged at 3,000 rpm, $5 \mathrm{~min}$. The pellet was discarded and the supernatant was centrifuged at 100,000g (45,000 rpm; TLA-45 rotor), 1 $\mathrm{hr}, 4^{\circ} \mathrm{C}$. Supernatant was the cytosol and the pellet was the membrane fraction. The cytosol fraction was preserved and the protein concentration determined. The cytosol containing equal amount of total protein was used for incubation with GSTfusion protein bound to GSH-Sepharose beads.

\subsubsection{0 purification of GST-fusion proteins}

buffer A: PBS, protease inhibitors with 0.1\%Triton X-100

buffer B: $50 \mathrm{mM}$ Tris- $\mathrm{Cl} \mathrm{pH}$ 8.0, $10 \mathrm{mM}$ reduced glutathione, adjusted to $\mathrm{pH}$ 8-8.5 with $\mathrm{NaOH}$ (prepared freshly)

A bacterial clone expressing the GST-fusion protein under the control of an inducible promoter was grown in a $10 \mathrm{ml} \mathrm{LB}$ preculture overnight. The culture was diluted 1:20 in fresh LB medium and grown for $3 \mathrm{~h}$ up to an optical density of 0.3-0.5 $(600 \mathrm{~nm})$. Protein expression was induced by adding $0.15 \mathrm{mM}$ IPTG to the culture, which was further grown for $3-5 \mathrm{hr}$ at $30{ }^{\circ} \mathrm{C}$. The cells were harvested at $(6,000$ rpm in a JA 10 rotor) and washed once with cold PBS. All the following steps were performed on ice. The pellet was resuspended in $4 \mathrm{ml}$ buffer $\mathrm{A}$, and lysed in french press with maximum 1000psi. The insoluble fraction was pelleted by centrifugation at $11,000 \mathrm{xg}(12,000 \mathrm{rpm}$ in a JA 20 rotor) and the supernatant transferred to a new tube. 50-100 $\mu$ l of glutathione-coupled sepharose beads (75\% suspension), corresponding to a bead volume of 100-125 $\mu$, were centrifuged at $1000 \mathrm{rpm}$ for 5 min in a tabletop centrifuge and washed twice with $0.5 \mathrm{ml}$ of buffer A. The beads were incubated with the soluble lysate fraction on a wheel at room temperature for $1 \mathrm{hr}$. After the incubation period, the sepharose was washed 5 times with buffer A. GST-fusion protein was eluted from the beads by adding $250 \mu \mathrm{l}$ of buffer D and incubating the sample for $15 \mathrm{~min}$ at room temperature. Elution was repeated at least 2 times. The eluted GST-fusion protein/GST alone was coupled to the beads 
and used to pull-down assay.

\subsubsection{Indirect immunofluorescence}

Cells were seeded on round coverslips in a 24-well plate 1-2 days in advance and grown to $50-70 \%$ confluency. All the incubation steps given were performed by filling the wells with $0.2 \mathrm{ml}$ of the respective solution unless indicated otherwise. Solutions were changed carefully, for the cells should neither be washed away nor become dry. The lid of the cell culture plate was closed during prolonged incubations and upon application of the secondary, fluorescent antibody the samples were protected from light.

\section{Fixation with para-formaldehyde:}

PFA reacts with nitrogen-containing groups and in effect crosslinks the proteins in a cell.

3\% para-formaldehyde (PFA):

The required amount of PFA was suspended in $1 / 5$ of the final volume, mixed with $50 \mu \mathrm{l}$ of $1 \mathrm{M} \mathrm{NaOH}$ per $\mathrm{g}$ PFA and dissolved by incubation at $60{ }^{\circ} \mathrm{C}$ for about 1 $\mathrm{hr}$ with intermittent mixing. The solution was always freshly prepared but can be stored for $1-2$ weeks at $4{ }^{\circ} \mathrm{C}$.

$0.5 \%$ saponin-PBS:

Prepared fresh from a $10 \%$ stock solution stored at $-20^{\circ} \mathrm{C}$

\section{Protocol}

Cells were washed twice with PBS and fixed with 3\% PFA for 20 min at room temperature. After two washes with PBS, aldehyde groups were blocked by 50 $\mathrm{mM} \mathrm{NH}_{4} \mathrm{Cl}$ for $10 \mathrm{~min}$ at room temperature. The cells were washed twice with PBS and permeabilised for $3 \times 5 \mathrm{~min}$ at room temperature with $0.5 \%$ saponin in PBS. After permeabilization, cells were wased once with PBS/saponin and incubated with primary antibody for $45 \mathrm{~min}-1 \mathrm{hr}$ at $37{ }^{\circ} \mathrm{C}$. Then cells were washed twice with PBS/saponin and incubated with $10 \%$ goat serum in PBS. After incubation, cells were washed once with PBS/saponin and incubated with secondary antibody coupled with flurochrome for $40 \mathrm{~min}$ at $37^{\circ} \mathrm{C}$. Then cells were washed twice with PBS and twice with water. The coverslips were carefully taken out with forceps, dried excess water and mounted upside-down over a drop of DAKO mounting medium on a glass slide. The slides were protected from light to dry overnight 
and sealed with nail-polish the next day. Confocal images were taken on Leica TCS SP2 AOBS laser scanning microscope.

\subsubsection{Trypsin sensitivity assay}

\section{preparation of adaptors from CCVs}

Cells were plated on $15 \mathrm{~cm}$ dishes to $80 \%$ confluency. Washed the cells twice with $20 \mathrm{ml}$ of ice-cold PBS. The cells were scrapped with a rubber police man in $1 \mathrm{ml}$ of MES buffer (0.1 M MES pH 6.6, 1mM EGTA, $0.5 \mathrm{mM} \mathrm{MgCl} 2,0.02 \%$ sodium nitrite). The cell suspension was frozen for minimum $20 \mathrm{~min}$ at $-80{ }^{\circ} \mathrm{C}$. The cells were thawed on ice and and lysed by gently passing through 22G needle for 10 times. Centrifuged the lysate at $1000 \mathrm{xg}\left(3000 \mathrm{rpm}\right.$ in Heraeus centrifuge) at $4{ }^{\circ} \mathrm{C}$. The pellet containing unlysed cells and the nuclear fraction was discarded and the postnuclear supernatant (PNS) was centrifuged at 7000xg (9000 rpm) for $40 \mathrm{~min}$ at 4 ${ }^{\circ} \mathrm{C}$. The resulting supernatant was subjected to centrifugation at $100,000 \mathrm{~g}$ (45000 $\mathrm{rpm}$ ) in an ultracentrifuge using TLA45 rotor at $4{ }^{\circ} \mathrm{C}$. The resulting pellet containing CCVs, was resuspended in $100 \mu \mathrm{l}$ of $1 \mathrm{M}$ Tris ( $\mathrm{pH}$ 8.0) and incubated for $20 \mathrm{~min}$ at room temperature, to strip the membrane bound proteins. Then, centrifuged the fraction at $100,000 \mathrm{~g}$ for $45 \mathrm{~min}$ at $4{ }^{\circ} \mathrm{C}$. The resulting supernatant was saved and its $\mathrm{pH}$ was adjusted to 7.0 and diluted such that the final concentration of Tris is 0.2 M. The concentration of protein was estimated by Biorad method.

\section{Partial Trypsin digestion}

Trypsin stock solution: $1 \mathrm{mg} / \mathrm{ml}$ in millipore grade water working concentration of trypsin: $0.01 \mu \mathrm{g} / \mu \mathrm{l}$ Trypsin inhibitor stock solution: $1 \mathrm{mg} / \mathrm{ml}$ Equal amount of total protein from CCVs were subjected to digestion with increasing concentrations of trypsin $(0 ; 0.25 ; 0.5 ; 0.75 ; 1.5 \mu \mathrm{g} / \mathrm{ml})$ by incubating the mixture at room temperature for 15 minutes. Immediately and quickly, trypsinisation was stopped, by adding trypsin-inhibitor to a final concentration of $20 \mu \mathrm{g} / \mathrm{ml}$ and placing the tubes in ice. The samples were boiled at $95{ }^{\circ} \mathrm{C}$ in $1 \mathrm{X}$ laemlli buffer containing $50 \mathrm{mM}$ DTT and placed on ice for 5 minutes and centrifuged for 5 minutes at 13000 rpm and subjected to 10\% SDS-PAGE, blotted onto nitro-cellulose membrane and probed with anti- $\gamma-1$ and anti- $\mu-1$ antibody. The signals were quantified by $2 \mathrm{D}$ densitometry. 


\subsubsection{Fluorescence recovery after photobleaching (FRAP)}

Cells were grown to 80\% confluency and transfected using FuGENE 6 (Roche Diagnostics, Penzberg, Germany). After $12 \mathrm{~h}$ cells received $5 \mathrm{mM}$ sodium butyrate for $18 \mathrm{~h}$. Cultures were confluent at the time of the experiment excluding differences due to cell cycle transissions. Hypertonic treatment was performed by incubating the cells in $0.2 \mathrm{M}$ sucrose for $30 \mathrm{~min}$ at $37{ }^{\circ} \mathrm{C}$. FRAP experiments were performed with a laser scanning confocal microscope (Leica TCS SP2, Heidelberg, Germany) using a $63 \mathrm{x}, 1.2$ N.A. plan apochromat water objective. The EGFP- $\gamma 1$-adaptin construct was excited with the $488 \mathrm{~nm}$ line of an Argon ion laser. Fluorescence was detected between 500 and $600 \mathrm{~nm}$. A single photobleaching run consisted of 10 prebleach and 150 postbleach frames. A defined region at the TGN was scanned for photobleaching once at $2 \mathrm{~mW}$ laser power (at back pupil of objective) for $1.3 \mathrm{~s}$ to reduce the fluorescence intensity to background level. The recovery of fluorescence was monitored by scanning the whole cell every $1.5 \mathrm{~s}$ at $0.15 \mathrm{~mW}$ laser power. All FRAP experiments were performed at $28{ }^{\circ} \mathrm{C}$ with opened pinhole for high signal-to-noise ratio. Images were analysed using Metamorph software (Universal Imaging, Philadelphia, USA). Fluorescence signals in the photobleached region were corrected for background. For reconstructing the recovery time series fluorescence in the bleached region of the TGN was normalized with respect to the fluorescence of a nearby unbleached region at each time point. This way we corrected for the loss of total fluorescence due to the bleaching in the TGN subregion as well as for the slight photobleaching during the collection of the recovery time series. Fluorescence recovery data are normalized with the measured prebleach intensity, $\mathrm{I}_{\text {pre }}$, and the intensity measured directly after photobleaching, $\mathrm{I}_{0}$, according to $\mathrm{I}_{n}(\mathrm{t})=\left(\mathrm{I}(\mathrm{t})-\mathrm{I}_{0}\right) /\left(\mathrm{I}_{p r e}-\mathrm{I}_{0}\right)$.

\subsubsection{Immunoelectron microscopy}

Ultrathin cryosections were prepared as described. Cells were fixed with $2 \%$ paraformaldehyde and $0.1 \%$ glutaraldehyde in $0.1 \mathrm{M}$ sodium phosphate buffer ( $\mathrm{pH} 7.4$ ) for $30 \mathrm{~min}$ at RT directly on cell culture dishes. Cells were postfixed with $4 \%$ para-formaldehyde and $0.1 \%$ glutaraldehyde for $2 \mathrm{hr}$ on ice and removed from the dishes with a cell scraper. After washing twice with PBS/0.02 M glycine, cells were embedded in $10 \%$ gelatin, cooled on ice and cut into small blocks. Blocks were infused with $2.3 \mathrm{M}$ sucrose overnight, mounted on small metal pins, frozen and stored in liquid nitrogen. Ultrathin sections were cut at $-110{ }^{\circ} \mathrm{C}$ using a diamond knife (Diatome) in an ultracryomicrotome (Leica, Bensheim, Ger), collected using a 1:1 mixture of 1.8\% methyl-cellulose and 2.3 M sucrose (Liou et al., 1996), and 
deposited on formvar- and carbon-coated grids. For immunolabelling, sections were incubated with antibodies against clathrin heavy-chain, $\gamma 1$-adaptin or LAMP-1 (additional bridging antibody rabbit anti-rat IgG) for $30 \mathrm{~min}$ followed by a $20 \mathrm{~min}$ incubation with protein A-gold ( 5 and $10 \mathrm{~nm}$ ). For double labelling experiments, sections were fixed for $5 \mathrm{~min}$ with $1 \%$ glutaraldehyde after incubations with the first antibody and protein A-gold. After quenching with glycine $(0.02 \mathrm{M})$ and blocking with BSA, sections were incubated with the second antibody and protein A-gold. BSA-gold endocytosis was done by incubating the cells in DMEM, 0.5\% BSA and 5.0 OD BSA-gold (G. Posthuma, University Utrecht, NL). Sections were contrasted with uranyl acetate/methyl-cellulose (Tokuyasu, 1978) for $10 \mathrm{~min}$ on ice, embedded in the same solution and examined with a Philips CM120 electron microscope (Philips, Berlin, Ger). Images were processed for presentation using Adobe Photoshop versions 5 and 7.

\subsubsection{Preparation of whole cell lysate from isolated adipocytes}

\section{Krebs-Ringer bicarbonate buffer(KRB)}

$12.5 \mathrm{mM}$ HEPES-NaOH (pH 7.4), $120 \mathrm{mM} \mathrm{NaCl}, 6 \mathrm{mM} \mathrm{KCl}, 1.2 \mathrm{mM} \mathrm{MgSO}$, $1 \mathrm{mM}$ $\mathrm{CaCl}_{2}, 1 \mathrm{mM} \mathrm{Na} \mathrm{HPO}_{4}, 10 \mathrm{mM} \mathrm{NaHCO} 3,10 \mathrm{mM}$ Glucose.

\section{Homogenization buffer}

20 mM Tris-HCl (pH 7.4), 1 mM EDTA, 255 mM sucrose.

For isolation of adipocytes, the epidydymal fat pads were excised from a mouse and used fresh. The tissue was carefully cut into mm-sized pieces with a scalpel. The minced tissue was incubated at $37{ }^{\circ} \mathrm{C}$ with gentle shaking for not more than $30-40 \mathrm{~min}$ in $2 \mathrm{ml}$ of $\mathrm{KRB}$ containing $10 \mathrm{mg} / \mathrm{ml}$ untreated bovine serum albumin and $10 \mathrm{mg}$ crude collagenase -Type II (sigma). The digested tissue was washed five times with $2 \mathrm{ml}$ of $\mathrm{KRB}$ by centrifugation at $600 \times \mathrm{g}_{\max }$ over $1 \mathrm{~min}$. During each wash step, the supernatant and pellet containing connective tissues were carefully removed by aspiration and care was taken not to aspirate the isolated adipocytes that float on top of the supernatant. The isolated adipocytes were washed twice with homogenization buffer at approx. $20^{\circ} \mathrm{C}$ by centrifugation as before. After washes, the cells were resuspended in $1 \mathrm{ml}$ of homogenization buffer and homogenizaion was done with 10 strokes in a teflon pestle homogenizer. The homogenate was centrifuged at $16,000 \times \mathrm{xg}_{\max }$ at $4{ }^{\circ} \mathrm{C}$ for $15 \mathrm{~min}$ in a cooling table-top centrifuge. the solidified fat cake was carefully removed and the supernatant was saved for further analysis. The total protein concentration was estimated by Bio-Rad assay and 100 $\mu \mathrm{g}$ of total protein were seperated in a SDS-PAGE, blotted onto membranes and 
decorated with appropriate antibodies and signals quantified using 2D densitometry. 



\section{Chapter 3}

\section{Results}

\subsection{Role of AP-1 in intracellular sorting of sortilin}

\subsubsection{Analysis of the intracellular distribution of sortilin in AP-1 deficient cells}

Sortilin is a member of a growing family of Vps10p-domain containing, multiligand type I receptors involved in intracellular sorting of various ligands. A recent study identified, that the cytoplasmic tail of sortilin contained bonafide intracellular sorting signals (Nielsen et al., 2001). The primary sequence of the cytoplasmic domain of sortilin is closely related to the cytoplasmic domain of cation-independent mannose-6-phosphate receptor (MPR300), with a conserved tyrosine-based endocytic motif and a di-leucine based Golgi to endosome transport motif. Sortilin was shown to be predominantly localized at the TGN and vesicles with an extensive co-localization with MPR300 (Petersen et al., 2001). Moreover, it was shown that the MPR300-sortilin tail chimeras were as effective as wildtype MPR300 in restoring intracellular transport of $\beta$-hexosaminidase in $\mathrm{mpr}^{-}$cells, indicating that sortilin cytoplasmic domain can substitute for the cytoplasmic domain of MPR300 in lysosomal sorting. The authors also reported that the sortilin cytoplasmic domain interacted with GGA2, a member of the GGA family of monomeric adaptors that are involved in the transport of cargo from the TGN to the endosome, thereby concluding that the transport of sortilin from the TGN to endosomes is mediated by GGA2 (Nielsen et al., 2001). Previous studies from our group, based on AP-1deficient mice ( $\mu 1 \mathrm{~A}$-adaptin 'knock-out') has demonstrated the involvement of AP-1 in the transport of MPR300. Since sortilin exhibits similar sorting characteristics as of MPR300, we were interested to know the fate of sortilin in AP-1-deficient cells.

As a first step, we analyzed the intracellular distribution of sortilin in AP-1- 
deficient MEFs (mouse embryonic fibroblasts) by indirect-immunofluorescence microscopy. $\mu 1 \mathrm{~A}-/$ - fibroblasts and control(ct) fibroblasts ( $\mu 1 \mathrm{~A}-/$ - fibroblasts ectopically expressing $\mu 1 \mathrm{~A}$ ), shown to rescue the $\mu 1 \mathrm{~A}$ deficient phenotype (Meyer et al., 2000) were used. The cells were plated on coverslips and after recovery, transiently transfected with sortilin(human full length construct in pcDNA3.1). The cells were fixed and permeabilized, 18 hours post-transfection, using the PFA fixation method and stained with either mouse anti- $\gamma 1$ adaptin; mouse anti-sortilin antibody and rabbit anti-MPR300 antibody(Fig. 3.1) or rat anti-LAMP1 antibody (Fig. 3.2).

As previously shown, in $\mu 1 \mathrm{~A} \mathrm{-/-} \mathrm{cells,} \gamma 1$ adaptin staining is lost or very weak at the TGN (Fig. 3.1 D) with more peripheral distribution of MPR300 (Fig. 3.1 E) compared to ct cells, where both show overlapping staining at the TGN (Fig. 3.1 C). The distribution of sortilin(Fig. $3.1 \mathrm{G}$ ) and MPR300 (Fig. $3.1 \mathrm{H}$ ) in ct cells was mainly peri-nuclear and overlapped extensively (Fig. 3.1 I). But in $\mu 1 \mathrm{~A}-/-$ cells, there was a remarkable distribution of sortilin mainly to peripheral structures(Fig. $3.1 \mathrm{~J}$ ) with dispersed staining, overlapping extensively with re-distributed MPR300 (Fig. 3.1 L). Since MPR300 is known to re-distribute to early endosomal structures in AP-1-deficient cells, this co-localization implies that sortilin is also re-distributed to early-endosomes in a similar way as MPR300.

Since sortilin staining appeared even more widely dispersed compared to MPR300 positive endosomes in $\mu 1 \mathrm{~A}$-/- cells, we co-stained sortilin also with LAMP1, a marker for late endosomes and lysosomes (Fig. 3.2). In ct cells, $\gamma 1$ adaptin (Fig. 3.2 A) and LAMP1(Fig. 3.2 B) are completely segregated in distinct structures (Fig. 3.2 C) serving as marker for the TGN and late endosomes/lysosomes respectively. In $\mu 1 \mathrm{~A} \mathrm{-/-} \mathrm{cells,} \mathrm{as} \mathrm{shown} \mathrm{in} \mathrm{Fig.} \mathrm{3.2(D,E} \mathrm{and} \mathrm{F),} \mathrm{sortilin} \mathrm{is} \mathrm{found} \mathrm{in}$ dispersed structures, which were LAMP1 negative implying that sortilin is not missorted to lysosomes. To validate whether AP-3 is involved in the sorting events of sortilin, we analyzed the steady-state distribution of sortilin in AP3-/- (mocha) cells which are deficient for $\delta$-3 adaptin. AP-3-/- cells transiently expressing sortilin were fixed as mentioned above and stained with mouse anti-sortilin antibody and rat anti-LAMP1 antibody. Sortilin stained predominantly in the peri-nuclear area(Fig. 3.2 G) and was completely segregated from LAMP1 positive structures(Fig. 3.2 I) implying that its steady-state distribution is unaltered in the absence of AP-3.

Endocytosed sortilin was shown to travel to the TGN showing little or no recycling (Nielsen et al., 2001). In $\mu 1 \mathrm{~A}$-/- cells it has been shown previously that the endosome-TGN transport of the MPRs is blocked leading to the accumulation of the MPRs in early-endosomes (Meyer et al., 2000). Since the steady-state localization of sortilin was redistributed to MPR300 positive early-endosomes in $\mu 1 \mathrm{~A}-/$ - cells, we were interested to know whether the endosome-TGN transport of sortilin is affected 


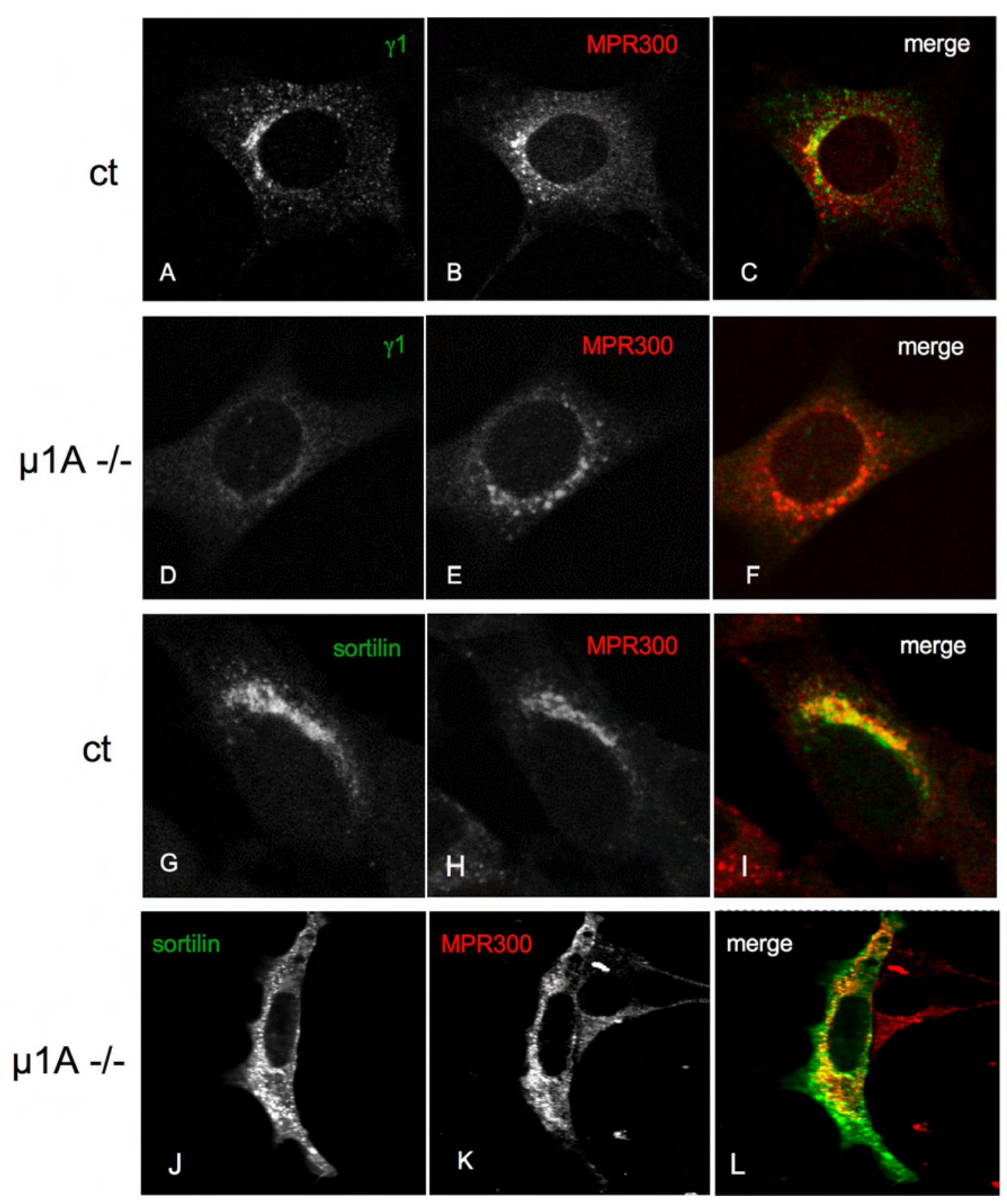

Figure 3.1: Intracellular localization of sortilin in AP-1 $(\mu 1 \mathrm{~A})$ deficient cells. Sortilin was transiently transfected in control(ct) and $\mu 1 \mathrm{~A}$ 'knockout' ( $\mu 1 \mathrm{~A}-/-)$ cells and fixed using $3 \%$ PFA, 18 hours post-transfection. Fixed cells were permeabilized with $0.5 \%$ saponin in PBS and incubated with antibodies against either $\gamma 1$ adaptin (anti-mouse IgG-Alexa 488 (green);A,D) and MPR300 (anti-rabbit IgG-Texas Red (red); B,E); merge ( $C$ and $F$ ) respectively or sortilin (anti-mouse IgG-Alexa 488 (green); G,J) and MPR300 (red;H,K); merge (I and L) respectively. 


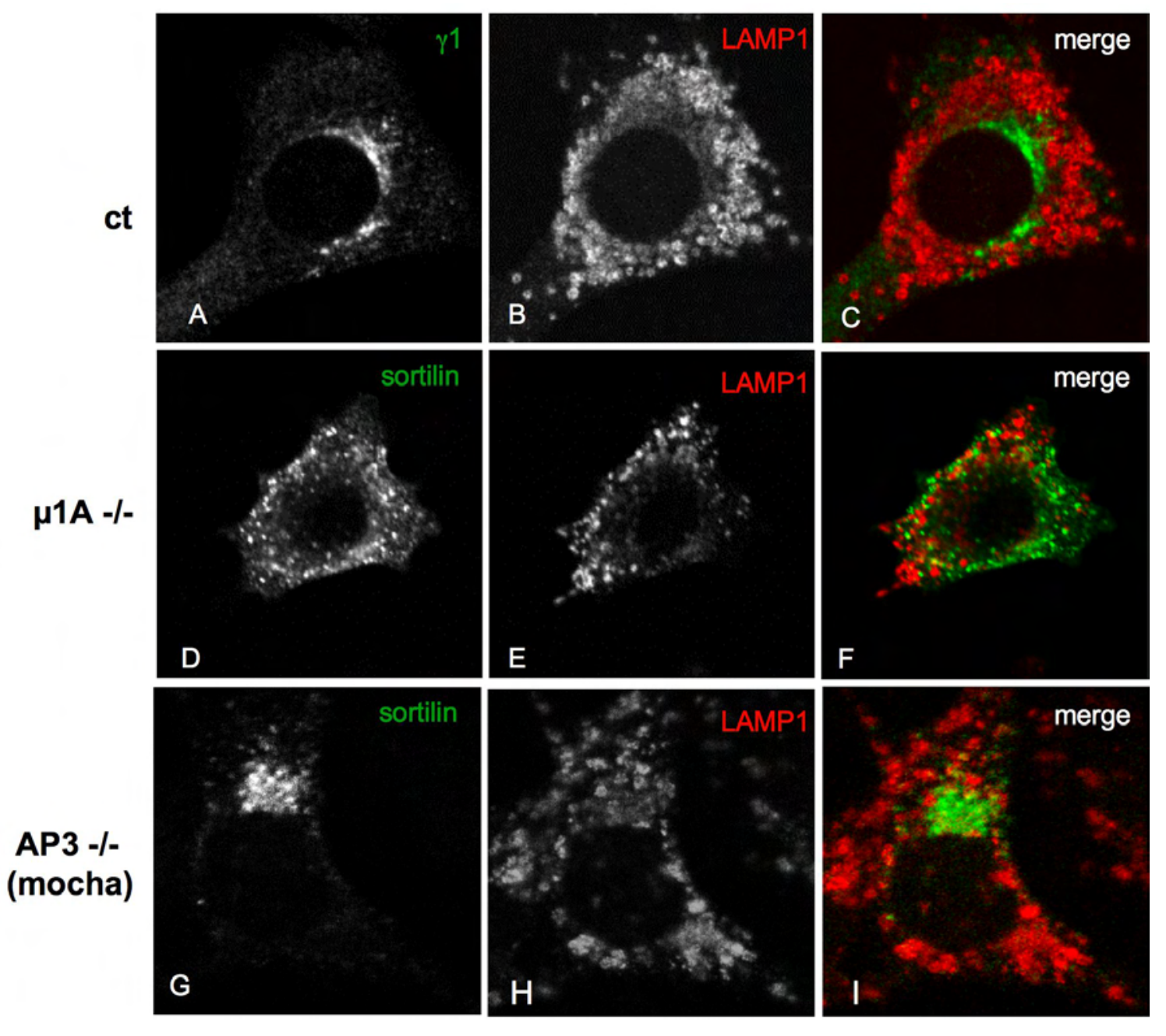

Figure 3.2: Intracellular localization of sortilin in AP-1-deficient and AP-3-deficient cells.Fixed cells were stained with antibodies against $\gamma$-1adaptin (green;A), sortilin (green;D and $G$ ) and LAMP1 (red;B,E and $H$ ). Merge represent the overlay of respective images ( $A, B: C$; $\mathrm{D}, \mathrm{E}: \mathrm{F} ; \mathrm{G}, \mathrm{H}: \mathrm{I})$.

in a similar way. For this purpose, we performed sortilin antibody endocytosis assay in control(ct) as well as in $\mu 1 \mathrm{~A}-/$ - cells and analyzed the intracellular distribution of sortilin by indirect-immunofluorescence microscopy. Control(ct) and $\mu 1 \mathrm{~A}$ 'knockout' ( $\mu 1 \mathrm{~A}-/-)$ cells were grown on coverslips and were transiently transfected with sortilin. At 18 hours post-transfection, the cells were allowed to endocytose sortilin antibody for $30 \mathrm{~min}$ at $37{ }^{\circ} \mathrm{C}$. Endocytosis was stopped immediately after $30 \mathrm{~min}$ by incubating the cells in ice and washing with ice-cold PBS to remove unbound antibodies. The cells were fixed and permeabilized and incubated with Alexa 488conjugated secondary antibody.

In control cells, endocytosed sortilin localized in the peri-nuclear region in a similar way as observed in steady-state (Fig. $3.3 \mathrm{~A}, \mathrm{~B}$ ). This indicates, that 
endocytosed sortilin reaches the TGN. But in $\mu 1 \mathrm{~A}-/$-cells, endocytosed sortilin did not display a peri-nuclear concentration, but was seen redistributed to peripheral structures as observed at steady-state (Fig. 3.3 C,D), indicating that the endosome to TGN transport is blocked upon AP-1 deficiency ( $\mu 1 \mathrm{~A}$ 'knock-out'), in a similar way, as shown for the MPRs (Meyer et al., 2000).

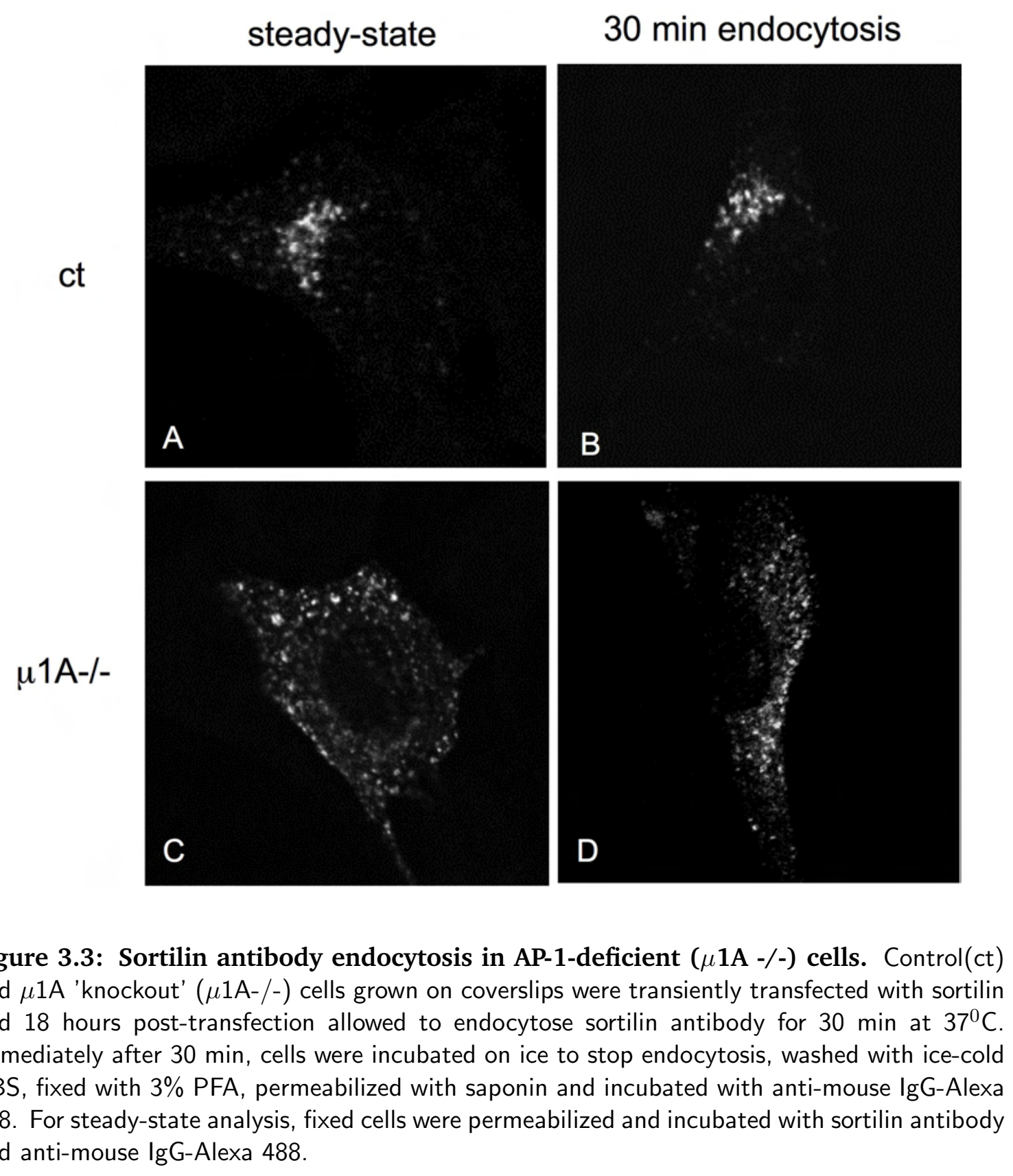




\subsubsection{Analysis of interaction between sortilin and AP-1}

\subsubsection{1 $\mathrm{Y} 2 \mathrm{H}$ analysis of sortilin-tail interaction with $\mu 1 \mathrm{~A}$}

The observed difference in the steady-state localization of sortilin in $\mu 1 \mathrm{~A}-/$ - cells prompted us to check whether the sortilin tail could to $\mu 1 \mathrm{~A}$. We adopted the $\mathrm{Y} 2 \mathrm{H}$ method to check for possible interaction, as this system has proven to be successful in identifying even weak interactions. Two different constructs of sortilin cytoplasmic domain(CD) were used in this analysis, because the sortilin CD contains two sorting motifs: a tyrosine-based motif and a di-leucine motif (Fig. 3.4). Both motifs are able to mediate binding to AP-1 and AP-2 complexes as it has been shown for MPR300 and many other proteins (Bonifacino and Traub, 2003).
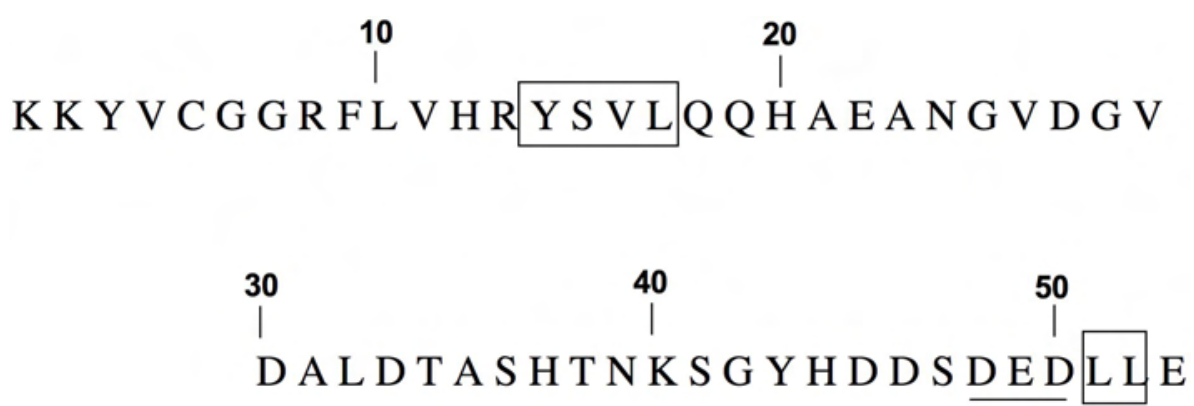

Figure 3.4: Primary structure of sortilin cytoplasmic domain(CD).The tyrosine-based and di-leucine based sorting motifs are highlighted by boxes and the acidic motif is underlined.

To this end, the cDNAs encoding the full length $\mathrm{CD}$ (sortilin $\mathrm{CD}$ ) and a truncated form lacking the tyrosine-based motif, but containing the di-leucine motif (sortilin $\mathrm{CD}(\Delta 1-30)$ ), were cloned into the bait vector (pGBT9) as a fusion to the Gal4 DNAbinding domain. As prey, the cDNA encoding the C-terminal domain (150-450 aa) of $\mu 1 \mathrm{~A}$ ( $\mu 1 \mathrm{~A} \mathrm{CT}$ ) that contains the sorting motif binding site, was cloned into the prey vector (pGADT7) as a fusion to the Gal4 activation domain. Since sortilin CD contains sorting motifs related to MPR300 and MPR46, cDNAs encoding the CDs of both the MPRs were also cloned into bait vectors and included in this analysis. Both the bait and the prey vectors were co-transformed into the yeast strain AH109 using the standard lithium-acetate transformation method. The co-transformants were allowed to grow in liquid medium and equal amount of exponentially growing cells were dotted in respective selection plates in the fashion as shown (Fig. 3.5) and incubated at $30{ }^{\circ} \mathrm{C}$ for a minimum of 3 to 5 days. The growth in the selection plates is a direct measure of activation of reporter genes and thereby is a measure of the strength of interaction. To test for the possibility of auto-activation of the reporter genes, the baits were co-transformed with empty prey vector and vice versa. 
As shown in Fig. 3.5, after 3 days of incubation, the observed growth of yeast clones corresponding to MPR300 CD and $\mu 1 \mathrm{~A} \mathrm{CT}$, but not in the respective control spot indicates a positive and strong interaction between MPR300 CD and $\mu 1 \mathrm{~A} C T$.

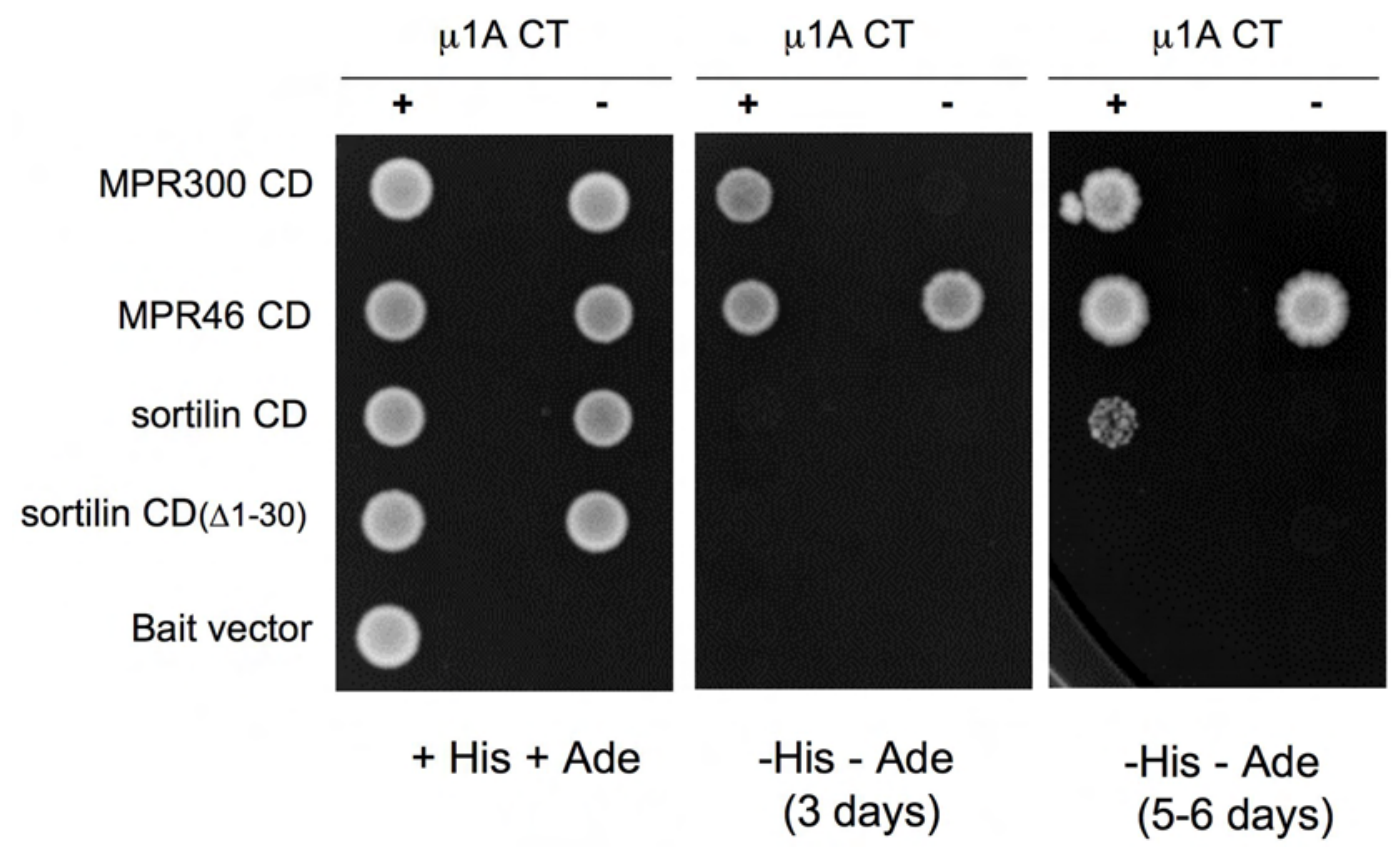

Figure 3.5: Y2H analysis of Interaction between sortilin CD and $\mu 1 \mathrm{~A}$. cDNAs encoding cytoplasmic domains(CD) of MPR300,MPR46 or sortilin (full length or truncated) were cloned into bait vector pGBT9 and cDNA encoding C-terminal domain(150-450 aa) of $\mu 1 \mathrm{~A}$ was cloned into prey vector pGADT7 to be expressed as a fusion with Gal4-DNA Binding domain and Gal4 Activation domain respectively. The bait and prey constructs were co-transformed into yeast strain $\mathrm{AH} 109$ in a combination as depicted in the figure and co-transformants were grown in liquid medium. Exponentially growing cells were diluted 1 to 10 and $5 \mu \mathrm{l}$ were dotted on selection plates (-Histidine-Adenine; -His-Ade) to test for activation of reporter genes and recorded after incubation for either 3 or 6 days. Growth in reporter selection plates indicates interaction. $(+)$ and (-) indicate the presence or absence of the prey $\mu 1 \mathrm{~A}$.

However, MPR46 CD exhibited auto-activation and so its interaction could not be validated. In the case of sortilin CD and $\mu 1 \mathrm{~A} C \mathrm{~T}$, growth was observed only after 5 to 6 days of incubation, implying a weak interaction. No interaction was observed between sortilin $\mathrm{CD}(\Delta 1-30)$ and $\mu 1 \mathrm{~A}$ CT. The above results indicate that sortilin CD interacts directly with $\mu 1 \mathrm{~A} \mathrm{CT}$ albeit weakly and this interaction is most likely, solely mediated by the tyrosine-based motif, as the sortilin CD lacking this motif failed to show even weak interaction. 


\subsubsection{In vitro analysis of sortilin and AP-1 interaction}

To confirm the interaction between sortilin $\mathrm{CD}$ and $\mu 1 \mathrm{~A}$ as observed by $\mathrm{Y} 2 \mathrm{H}$ interaction study, sortilin CD- $\mu 1 \mathrm{~A}$ interaction was tested in a GST-pull down assay using GST-sortilin CD fusion proteins and cytosol preparations from control and $\mu 1 \mathrm{~A}$ -/- cells. Sortilin $C D$ and sortilin $C D(\Delta 1-30)$ cDNAs were cloned into the vector pGEX2T through BamHI and EcoRI sites to express N-terminally fused GST fusion proteins in E.coli BL21(DE3).

Purification of the fusion proteins from bacterial lysate were done by binding the fusion proteins or GST alone to glutathione sepharose 4B and subsequent elution with reduced glutathione. The purity of the GST-fusion proteins were checked by loading $25 \mu \mathrm{g}$ of the purified proteins on a 15\% SDS-PAGE and visualizing after coomassie staining. As shown, the purified fusion proteins were pure enough to be used for further pulldown assays (Fig. 3.6).

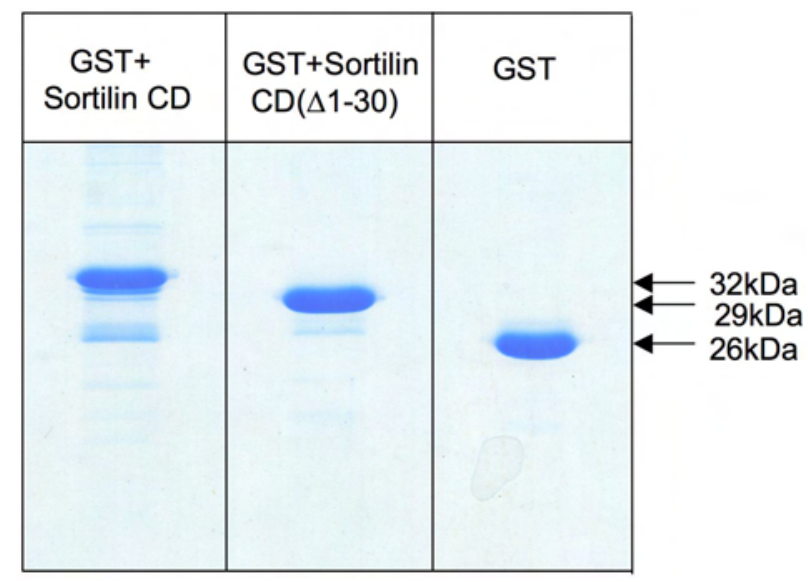

Figure 3.6: Purification of GST-Sortilin fusion proteins for pull down assay. GSTsortilin fusion proteins or GST alone from bacterial lysate were bound to Glutathione sepharose $4 \mathrm{~B}$, washed and eluted with elution buffer containing $10 \mathrm{mM}$ reduced glutathione. $25 \mu \mathrm{g}$ from each purified fraction were loaded on a 15\% SDS-PAGE and stained with coomassie-blue. Their respective molecular sizes are indicated.

For preparation of cytosol fractions from control and $\mu 1 \mathrm{~A}-/$ - cells, the cells were plated in $10 \mathrm{~cm}$ plates and when sub-confluent, harvested by scrapping in ice-cold lysis buffer that contains $0.5 \%(\mathrm{v} / \mathrm{v})$ Triton X-100. The cells were then lysed by sonication and the cytosol was prepared using the protocol as described in the materials and methods. Cytosol at a total protein concentration of $1.5 \mathrm{mg} / \mathrm{ml}$ in lysis buffer was added to $150 \mu \mathrm{g}$ of GST-fusion proteins or GST alone pre-bound to $100 \mu \mathrm{l}$ of glutathione sepharose beads and incubated overnight at $4{ }^{\circ} \mathrm{C}$ with endover-end rotation. After the incubation period, the flow-through(FT) was collected, beads were washed five times with $100 \mu$ of lysis buffer and then eluted with 10 
$\mathrm{mM}$ reduced glutathione and pooled the eluate fractions(E). 5\% of FT and 35\% of Eluate(E) fractions were seperated on a 7.5\% SDS-PAGE and blotted on PVDF membranes and tested for $\gamma 1$ adaptin (Fig. 3.7). It has been demonstrated that upon $\mu 1 \mathrm{~A}$-deficiency, protein levels of $\gamma 1$ and $\sigma 1$ adaptins are decreased (Meyer et al., 2000). Therefore the signal for $\gamma 1$ adaptin in the SM fraction from $\mu 1 \mathrm{~A}-/$ - cytosol is reduced to $70 \%$.

GST-Sortilin CD efficiently pulled down AP-1 from the control(ct) cytosol compared to GST-Sortilin $\mathrm{CD}(\Delta 1-30)$ that lacks the tyrosine-based motif (Fig. 3.7). Yet a weak signal for $\gamma 1$ was observed in the pull-down fraction of GST-Sortilin $\operatorname{CD}(\Delta 1$ 30) which means that the interaction is not completely lost. This result together with the $\mathrm{Y} 2 \mathrm{H}$ results, indicates that the interaction between GST-Sortilin CD and AP-1 is mediated by the tyrosine-based motif that binds to the $\mu 1 \mathrm{~A}$ subunit.

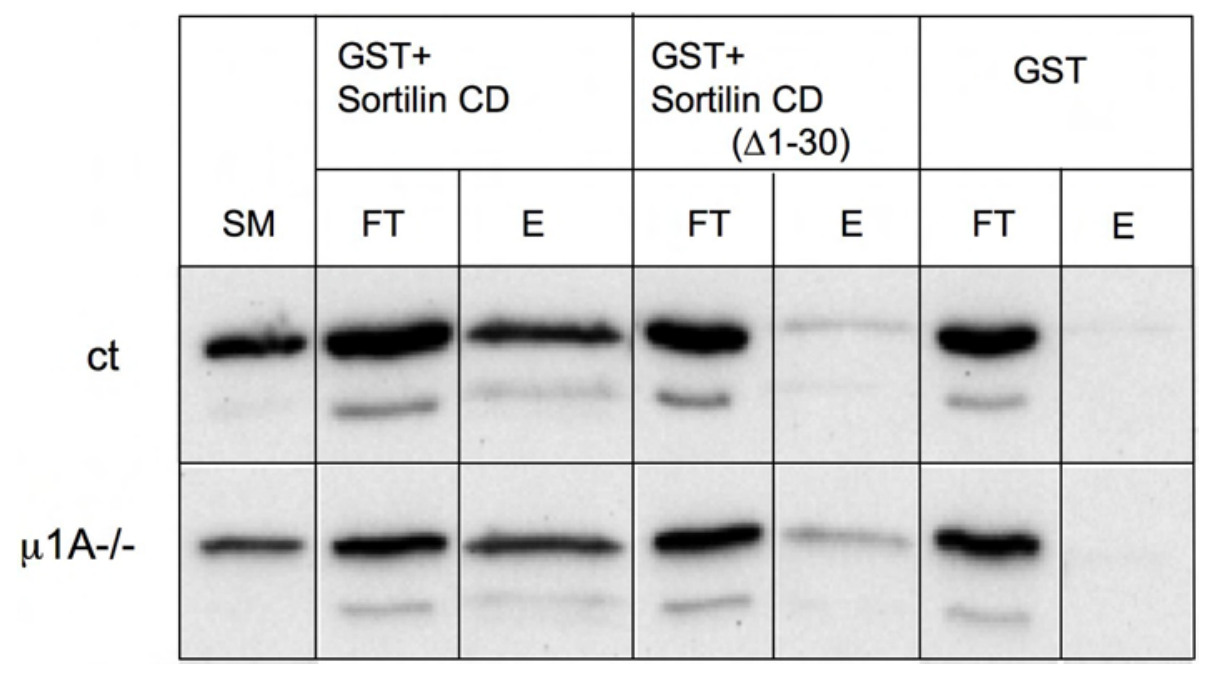

Figure 3.7: In vitro interaction of sortilin and AP1. Cytosol from ct or $\mu 1 \mathrm{~A}-/-$ cells at a total protein concentration of $1.5 \mathrm{mg} / \mathrm{ml}$ were added to $150 \mu \mathrm{g}$ each of GST-Sortilin CD, GSTSortilin $\mathrm{CD}(\Delta 1-30)$ or GST alone bound to $100 \mu \mathrm{l}$ of GSH-sepharose beads. After over night incubation, the flow-through(FT) was collected, beads washed 5 imes with lysis buffer and the bound fractions were eluted with $10 \mathrm{mM}$ reduced glutathione and the eluate $(\mathrm{E})$ fractions pooled. $1 \%$ of starting material(SM), $5 \%$ of FT and $35 \%$ of $E$ fractions were loaded on a $7.5 \%$ SDS-PAGE and blot was decorated with anti- $\gamma$-1adaptin antibody (representative of two experiments).

Surprisingly, similar results were observed when cytosol from $\mu 1 \mathrm{~A}-/-$ cells was used for pull-down assays (Fig. 3.7). Apparently GST-Sortilin CD was able to pull down $\gamma 1$-adaptin indicating that sortilin could bind to $\gamma 1$, or most likely to a $\gamma 1$ $\sigma 1$ hemi-complex, independent of $\mu 1 \mathrm{~A}$. It has been shown that a trimeric complex consisting of a $\gamma 1-\sigma 1-\beta 1$ adaptin still exists in $\mu 1 \mathrm{~A}-/$ - fibroblasts (Meyer et al., 2000). However, this trimeric complex is non-functional in protein sorting, because it does not bind to membranes in vivo, leading to a loss of $\gamma 1$ adaptin staining 
in the peri-nuclear area, but with a weak cytoplasmic staining, as also shown in (Fig. 3.1 E). So, it is possible that sortilin CD interacts with the cytosolic $\gamma 1-\sigma 1$ $\beta 1$ complex present in $\mu 1 \mathrm{~A}-/$ - cells. Recent studies have shown that $\gamma 1-\sigma 1$ hemicomplex interacts with acidic di-leucine motif present in the cytosolic tails of HIVNef and LIMP-II (Janvier et al., 2003) and so one can expect that the interaction between sortilin and the $\gamma 1-\sigma 1$ hemi-complex could be mediated by the acidic dileucine motif present in sortilin $\mathrm{CD}$, although only a weak signal for $\gamma 1$ was observed in the pull-down fraction of GST-Sortilin $C D(\Delta 1-30)$. One possible explanation to this discrepancy could be that the acidic di-leucine motif in GST- Sortilin $\operatorname{CD}(\Delta 1$ 30) was not completely accessible for interaction due to steric hinderance or due to the requirement of a post-translational modification. It is also possible that binding of the di-leucine motif is mediated by the $\beta 1$-adaptin core domain. The interaction observed between sortilin and AP-1 was authentic since no interaction was observed when GST alone was used for the pulldown with control(ct) or $\mu 1 \mathrm{~A}-/$ - cytosol (Fig. 3.7).

\subsubsection{Analysis of Interaction between sortilin and $\gamma 1-\sigma 1$ hemi-complex}

\subsubsection{Yeast-3-Hybrid analysis}

To elaborate on the In vitro interaction studies, we decided to verify a direct interaction between sortilin and the $\gamma 1-\sigma 1$ hemi-complex using the Yeast-3-Hybrid(Y3H) method. $\mathrm{Y} 3 \mathrm{H}$ is a valuable tool to check the interaction between three proteins, wherein the interaction between two chimeric proteins requires the presence of a third protein.

The cDNAs encoding the full length sortilin CD or sortilin CD ( $\Delta 1-30)$ were cloned into one of the two cloning sites of the bait vector pBridge to be expressed as a fusion with the Gal4-DNA binding domain. Full length cDNAs encoding different isoforms of $\sigma 1$ adaptin, $\sigma 1 \mathrm{~A}, \sigma 1 \mathrm{~B} 1$ - its splice variants $\sigma 1 \mathrm{~B} 2, \sigma 1 \mathrm{~B} 3$ and $\sigma 1 \mathrm{C}$ were cloned into the second cloning site of pBridge vector to be expressed under the control of MET25 promoter. $\sigma 1$-adaptins are therefore only expressed, if the yeast is grown in medium lacking methionine and are repressed in the presence of $1 \mathrm{mM}$ methionine. The cDNA encoding $\gamma 1$ adaptin was cloned into the prey vector to be expressed as a fusion with the Gal4-activation domain. Bait and prey constructs were co-transformed into yeast strain CG1945 using lithium acetate transformation protocol. The co-transformants were grown in liquid medium containing $1 \mathrm{mM}$ methionine, then equal amount of cells from exponentially growing cultures were dotted onto medium lacking histidine and methionine (-His,-Met), incubated for 3 to 5 days and results were recorded. As shown in Fig. 3.8 A, sortilin CD and 
A

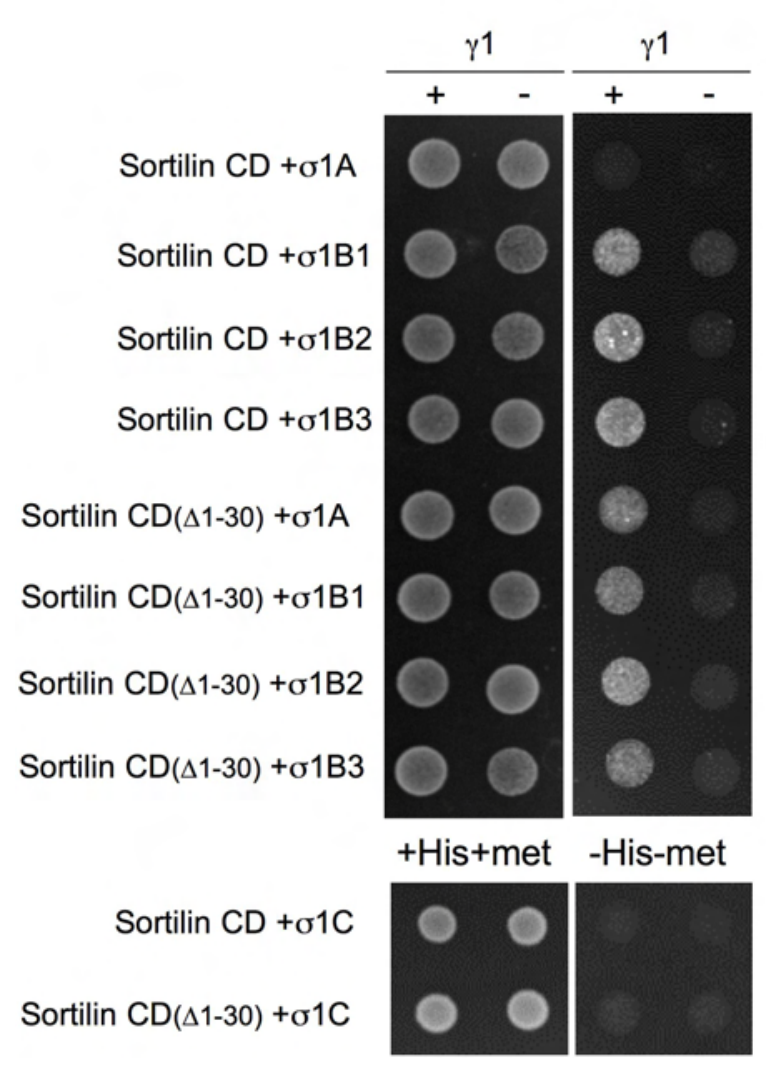

B

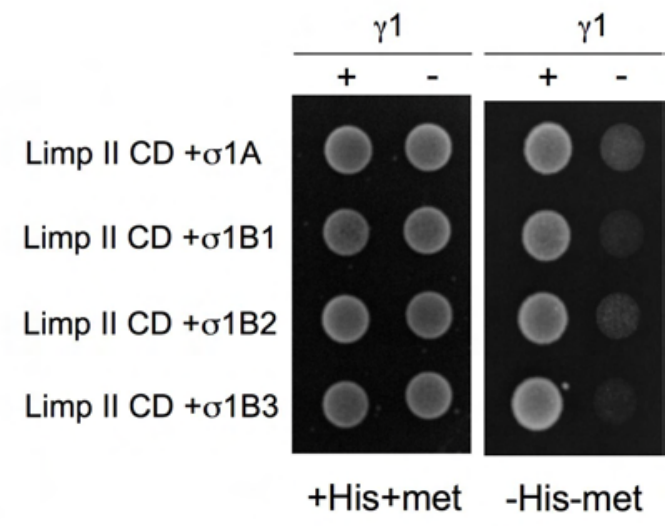

Figure 3.8: Y3H analysis of the interaction between sortilin and $\gamma 1-\sigma 1$ hemicomplex.(A) sortilin $\mathrm{CD} /$ sortilin $\mathrm{CD}(\Delta 1-30)$ or (B) LIMP II cytosolic tail as a fusion of Gal4DNA binding domain and different isoforms of $\sigma 1$ adaptin viz., $\sigma 1 \mathrm{~A}, \sigma 1 \mathrm{~B} 1$ and its splice variants $\sigma 1 \mathrm{~B} 2, \sigma 1 \mathrm{~B} 3$ and $\sigma 1$, were expressed from the bait vector pBridge. The $\sigma 1$ adaptins were under the control of MET25 promoter;expressed when the yeast is grown in medium lacking methionine and repressed in the presence of $1 \mathrm{mM}$ methionine. $\gamma 1$ adaptin was expressed as a fusion of Gal4-activation domain from the prey vector pGADT7. Both the bait and prey constructs were co-transformed into yeast strain CG1945. The co-transformants were grown in liquid medium containing $1 \mathrm{mM}$ methionine and equal $\mathrm{OD}$ of cells from exponentially growing culture were dotted onto medium lacking histidine and methionine (-His,-Met); incubated for 3 to 5 days and interaction was evaluated according to the growth of yeast in the selection medium.

sortilin $\mathrm{CD}(\Delta 1-30)$ interacted with the $\gamma 1-\sigma 1$ hemi-complexes. Since, the truncated sortilin $\mathrm{CD}(\Delta 1-30)$, lacking the tyrosine-based motif, but containing the di-leucine motif was sufficient for the interaction, it can be concluded that the interaction between sortilin and the $\gamma 1-\sigma 1$ hemi-complex is mediated by the di-leucine motif. Surprisingly, this interaction was restricted only to the hemi-complex containing the $\sigma 1 \mathrm{~B}$ isoform and its splice variants $\sigma 1 \mathrm{~B} 2$ and $\sigma 1 \mathrm{~B} 3$, but little to $\sigma 1 \mathrm{~A}$ and no interaction with and $\sigma 1 \mathrm{C}$ containing hemi-complexes was detected. Interestingly no interaction was detectable with sortilin $\mathrm{CD} \gamma 1-\sigma 1 \mathrm{~A}$, however sortilin $\mathrm{CD}(\Delta 1$ - 
30) containing only the di-leucine motif did show a weak $\gamma 1-\sigma 1 \mathrm{~A}$ binding. This indicates that aa 1-30 of sortilin CD prevent binding of the membrane distal dileucine motif with a $\gamma 1-\sigma 1 \mathrm{~A}$ hemi-complex, presumably by adopting a secondary structure preventing di-leucine motif binding to the hemi-complex. For example, the acidic residues preceding the di-leucine motif could bind to membrane proximal basic residues. This also indicates that the affinities of sortilin to $\gamma 1-\sigma 1 \mathrm{~B}$ hemicomplexes are higher than the $\gamma 1-\sigma 1 \mathrm{~A}$ binding affinities, because they are able to bind to the full-length tail.

Further, it was interesting to note different affinities to the $\sigma 1 \mathrm{~B}$ splice variants. $\sigma 1 \mathrm{~B} 1$ containing hemi-complex showed strong interaction with sortilin $\mathrm{CD}$ and $\sigma 1 \mathrm{~B} 2$ containing hemi-complex showed strong interaction with sortilin $\mathrm{CD}(\Delta 1-30)$ as assessed from the difference in growth rate of the yeast in corresponding spots (Fig. $3.8 \mathrm{~A}$ ). Absence of growth in yeast containing the baits and empty prey vector or vice versa served as controls for auto-activation. In a seperate experiment, we checked for interaction between sortilin and $\gamma 1$ subunit alone, but no interaction was observed. The interaction observed only with the hemi-complex implies that both subunits are necessary for interaction with sortilin. The low affinities of the interactions observed are also demonstrated by the absence of detectable $\beta$ galactosidase activities in a colony filter-lift assay and by complete inhibition of growth in the presence of $0.5 \mathrm{mM} 3$-AT, an inhibitor of the HIS3 gene product.

Since it was reported in a study (Janvier et al., 2003), that the acidic di-leucine motif of the type D/EXXXL(L/I) present in the cytoplasmic tails of HIV-Nef and LIMPII mediated their interaction with the $\gamma 1-\sigma 1 \mathrm{~A}$ hemi-complex, we were interested to know whether the differential interaction, observed in our interaction studies with the sortilin tail (Fig. 3.8 A), is maintained with other di-leucine motif containing proteins. For this purpose we chose to test for the interaction of LIMP-II cytosolic tail with $\gamma 1-\sigma 1$ hemi-complexes. All four different $\gamma 1-\sigma 1$ hemi-complexes interacted with the LIMP-II cytosolic tail (Fig. 3.8 B) with no apparent difference in specificity or affinity. However, the affinities appear to be higher than those of sortilin, indicating that only low-affinity binding allows to discriminate between the family of $\gamma 1-\sigma 1$ hemi-complexes.

\subsubsection{In vitro interaction of sortilin with AP-1 from $\sigma 1 \mathrm{~B}-/-$ cells}

After observing an interaction between sortilin and the $\gamma 1-\sigma 1 \mathrm{~B}$ hemi-complex at the Y3H level, we performed GST pull-down assay to see whether we can observe the interaction in vitro. The $\sigma 1 \mathrm{~B}$-deficient mouse embryonic fibroblasts were obtained from a $\sigma 1 \mathrm{~B}$ 'knock-out' mouse generated in our lab (Riel, 2004). GST-Sortilin CD, 


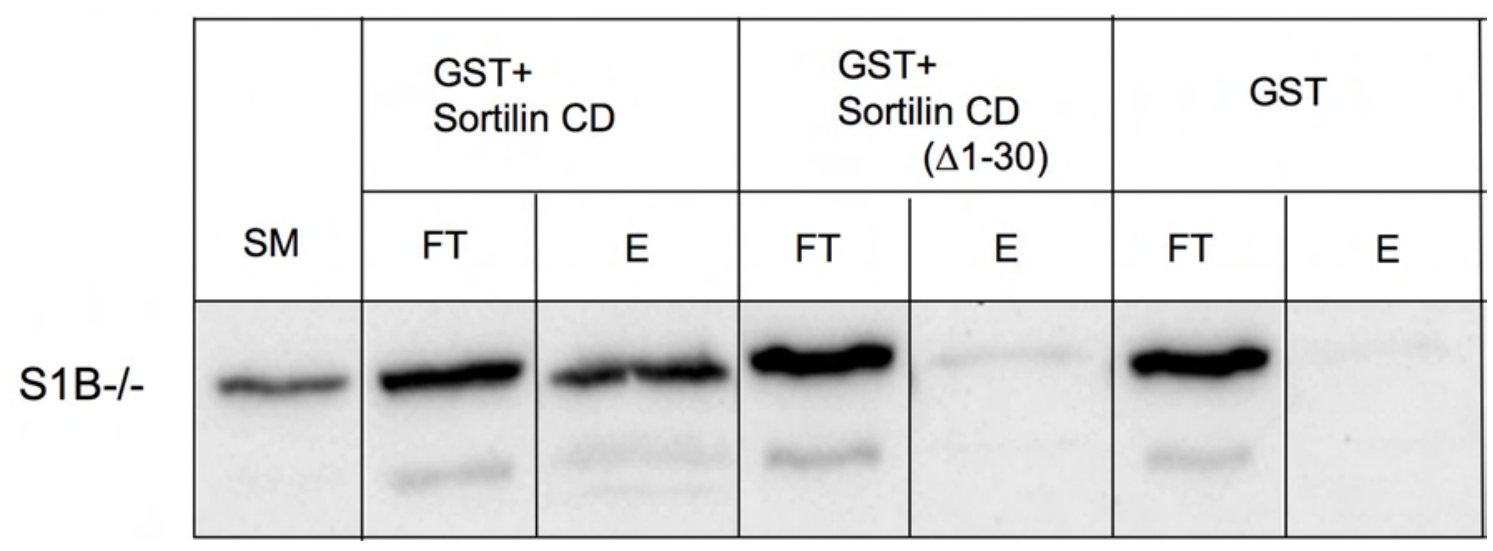

Figure 3.9: In vitro interaction of sortilin and AP-1 in $\sigma 1 \mathrm{~B}-/-$ cells. GST-Sortilin or GST alone bound to beads were incubated with cytosol prepared from $\sigma 1 \mathrm{~B}-/-\mathrm{MEFs}$. The flowthrough(FT) was collected before washing the beads 5 times with lysis buffer and finally eluted with reduced Glutathione, $\mathrm{pH}(8.0)$. $1 \%$ of the starting material(SM), $5 \%$ of flowthrough(FT) and $35 \%$ of the Eluate(E) were loaded on a $7.5 \%$ SDS-PAGE and the blot was probed with antibody against $\gamma 1$ adaptin (representative of two experiments).

GST-Sortilin $C D(\Delta 1-30)$ or GST alone bound to GSH-sepharose beads and cytosol prepared from $\sigma 1 \mathrm{~B}$-/ - fibroblasts were incubated overnight at $4{ }^{\circ} \mathrm{C}$ and the pulldown assay was performed essentially in the same way as described in the previous section (3.1.2.2).

GST-Sortilin CD was able to pull-down AP-1 from the $\sigma 1 \mathrm{~B}-/$ - cytosol, but no interaction with either GST-Sortilin $\mathrm{CD}(\Delta 1-30)$ or GST alone (Fig. 3.9), was detected. This was not surprising, because sortilin CD is able to bind to $\mu 1 \mathrm{~A} \mathrm{CT}$ through the tyrosine-based motif (Fig. 3.5). Thus this interaction appears to be responsible for the pull-down of AP-1 by sortilin CD. In all pull-down experiments, the contribution of the di-leucine motif is barely detectable, confirming the lowaffinity interaction observed in the $\mathrm{Y} 3 \mathrm{H}$ assay. This observation substantiates the idea that sortilin CD interacts with AP-1 via both the tyrosine-based and acidic dileucine motif independent of each other.

\subsubsection{Analysis of steady-state distribution of Sortilin in $\sigma 1 \mathrm{~B}-/-$ cells}

Having observed from previous experiments that sortilin could interact with $\gamma 1$ $\sigma 1 \mathrm{~B}$ hemi-complex, we were interested to check for the steady-state distribution of sortilin in $\sigma 1 \mathrm{~B}$-/- fibroblasts by indirect immunofluorescence microscopy. Wildtype (WT) and $\sigma 1 \mathrm{~B}-/-$ MEFs transiently expressing sortilin were fixed, permabilized according to the PFA fixation method and incubated with mouse anti-sortilin and rabbit anti-MPR300/rat anti-LAMP1. The secondary antibodies conjugated with 


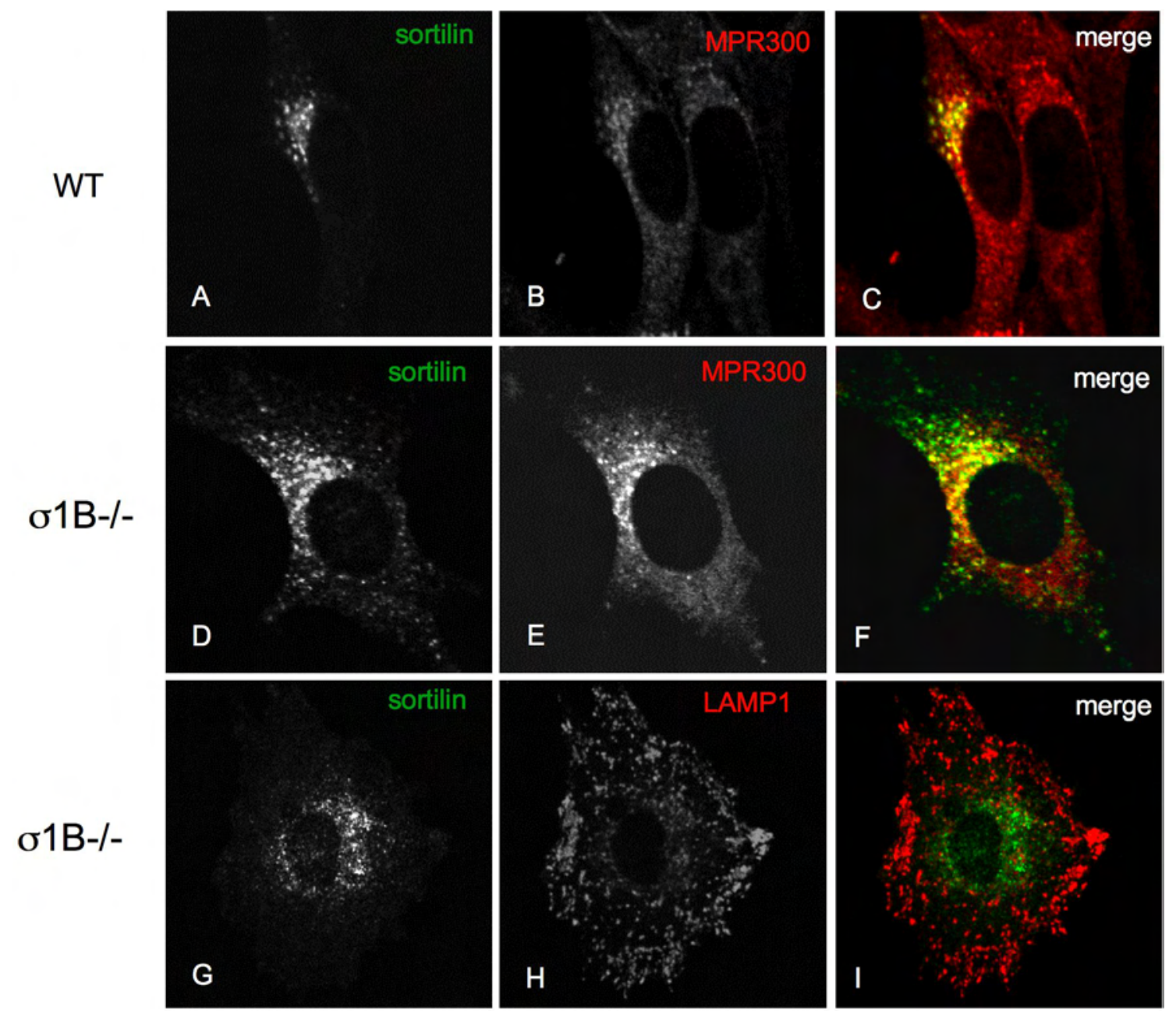

Figure 3.10: Steady state distribution of sortilin in $\sigma 1 \mathrm{~B}-/-$ MEFs . Cells transiently expressing sortilin were fixed using 3\% PFA and permeabilized by $0.5 \%$ saponin in PBS, $\mathrm{pH}(7.4)$ and incubated with antibodies against sortilin (green; $A, D, G$ ), MPR300 (red;B,E) and LAMP1(red,H). Merge C, F and I represent the overlay of images $A \& B$, D\&E and G\&H respectively.

fluorescent probes used were goat anti-mouse Alexa 488 for sortilin, goat anti-rabbit Texas Red and goat anti-rat Cy3 for LAMP1.

In $\sigma 1 \mathrm{~B}-/-$ cells, sortilin was found to be predominantly localized in the perinuclear region and showed high overlap with the distribution of MPR300 (Fig. 3.10 D,E and F) as observed in wild-type cells (Fig. 3.10 A,B and C). This indicates, that the steady state distribution of sortilin was unaltered upon $\sigma 1 \mathrm{~B}$ deficiency. When costained along with LAMP1 a marker for late endosomes/Lysosomes, sortilin staining was completely segregated from LAMP1 staining (Fig. 3.10 G,H and I). 


\subsubsection{Analysis of intracellular distribution of Sortilin sorting- motif mutants}

To further delineate the interactions observed between sortilin and AP-1 and to characterize the sorting motifs that mediate this interaction, we looked for the intracellular distribution of sortilin CD mutants in control (ct) and $\sigma 1 \mathrm{~B}-/-\mathrm{MEF}$ by indirect immunofluorescence microscopy. For this purpose, Interleukin-2 Receptor$\alpha(\operatorname{IL} 2 \mathrm{R} \alpha)$-sortilin CD mutant chimeras were used, which were obtained from Prof. C.M.Petersen's lab (Denmark) as part of a collaboration. Sortilin CD mutants were created by either deleting certain selected potential residues or exchanging them by alanine, or by aspartate in the case of S47. These sortilin CD mutants were combined with the extracellular and transmembrane segments of IL2R $\alpha$ to create IL2R-Sor CD mutants (Nielsen et al., 2001). The interleukin-receptor luminal domain allowed immunofluorescence microscopy analysis with a commercially available interleukin-receptor antibody. In our study, these IL2R-Sor CD mutants were transiently transfected into either Control(ct) or $\sigma 1 \mathrm{~B}-/-$ MEFs. The cells were fixed after 18 hours of transfection using PFA fixation method and incubated with mouse anti-IL2R. The bound primary antibody was then decorated with anti-mouse IgG-Alexa488.

In control cells and in $\sigma 1 \mathrm{~B}-/-$ cells, IL2R-sortilin CD-wt was predominantly localized in the peri-nuclear compartments in agreement with the published study (Nielsen et al., 2001) (not shown). As shown, IL2R-sortilin CD mutants expressed in control cells and in $\sigma 1 \mathrm{~B}-/$ - cells (Fig. 3.11 and Fig. 3.12, respectively), exhibited a similar pattern of steady-state distribution. Both IL2R-sortilin CD-Y14A and IL2R-sortilin CD-Y14A/L17A were re-distributed to peripheral structures, a pattern similar to that observed in $\mu 1 \mathrm{~A}-/$ - cells. This indicates that the tyrosine residue is most important for $\mu 1 \mathrm{~A}$-dependent transport between the endosomes and the Golgi. In contrast, IL2R-sortilin CD- $\Delta$ LL exhibited an absolute localization at the perinuclear compartment with little or no distribution in vesicular structures in contrast to sortilin-wt in control cells (fig. $3.1 \mathrm{G}$ ). This could indicate a possible blockade in the exit of IL2R-Sortilin CD- $\Delta L L$ receptor at the TGN, implicating the significance of di-leucine in transport of the receptor from the TGN. The mutant sortilin CDY14A/L17A $+\Delta L L$ exhibited a mixed pattern with prominent localization at the plasma membrane and also with considerable peri-nuclear and vesicular staining. All other sortilin mutants: sortilin CD-S47D, -S47A, -G42A/Y43A, -D45A/D46A and -D48A/E49A/D50A showed a predominant localization at the peri-nuclear compartment, a pattern as exhibited by sortilin-wt in control cells, indicating that they are dispensible for the intracellular sorting of sortilin. 

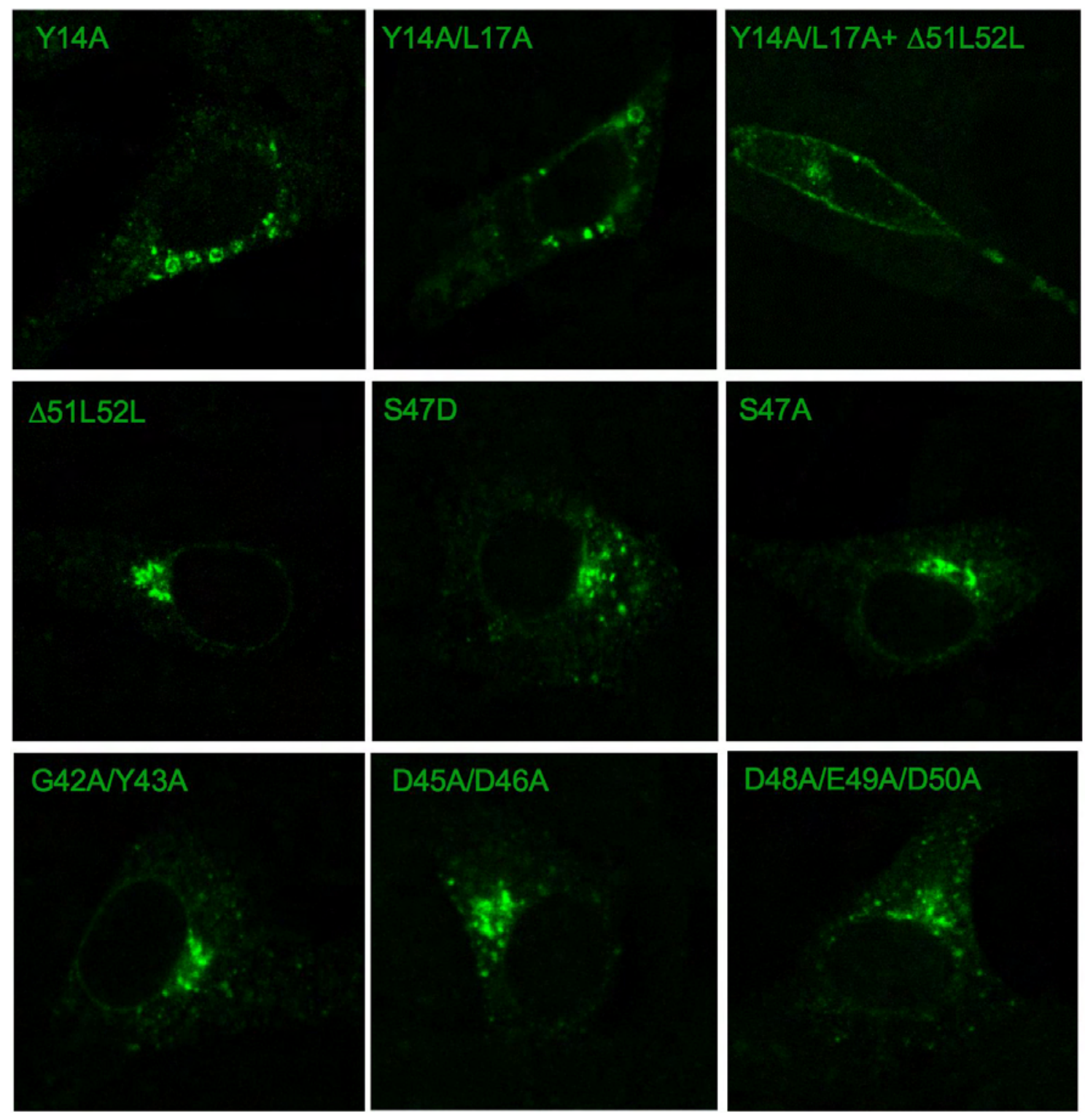

Figure 3.11: Steady state distribution of IL2R-Sor CD mutants in Control cells. IL2RSor CD mutants indicated in their respective images were transiently expressed in control cells and the cells were fixed, permeabilized and incubated with primary antibodies against IL2R and then decorated with secondary antibodies conjugated with Alexa488.

These results indicate that the phenotype observed in $\mu 1 \mathrm{~A}-/$ - cells is attributable to the tyrosine-based sorting motif as supported by sortilin and AP-1 interaction studies presented in this study. Analysis of the steady-state distributions of these mutants in MEF lacking the $\sigma 1 \mathrm{~B}$-adaptin did not reveal differences to wt cells. Thus, the tyrosine-based motif apparently required for endosome to TGN transport, can mediate sortilin sorting by AP- $1 / \sigma 1 \mathrm{~A}$ as well as AP- $1 / \sigma 1 \mathrm{~B}$ complexes. 


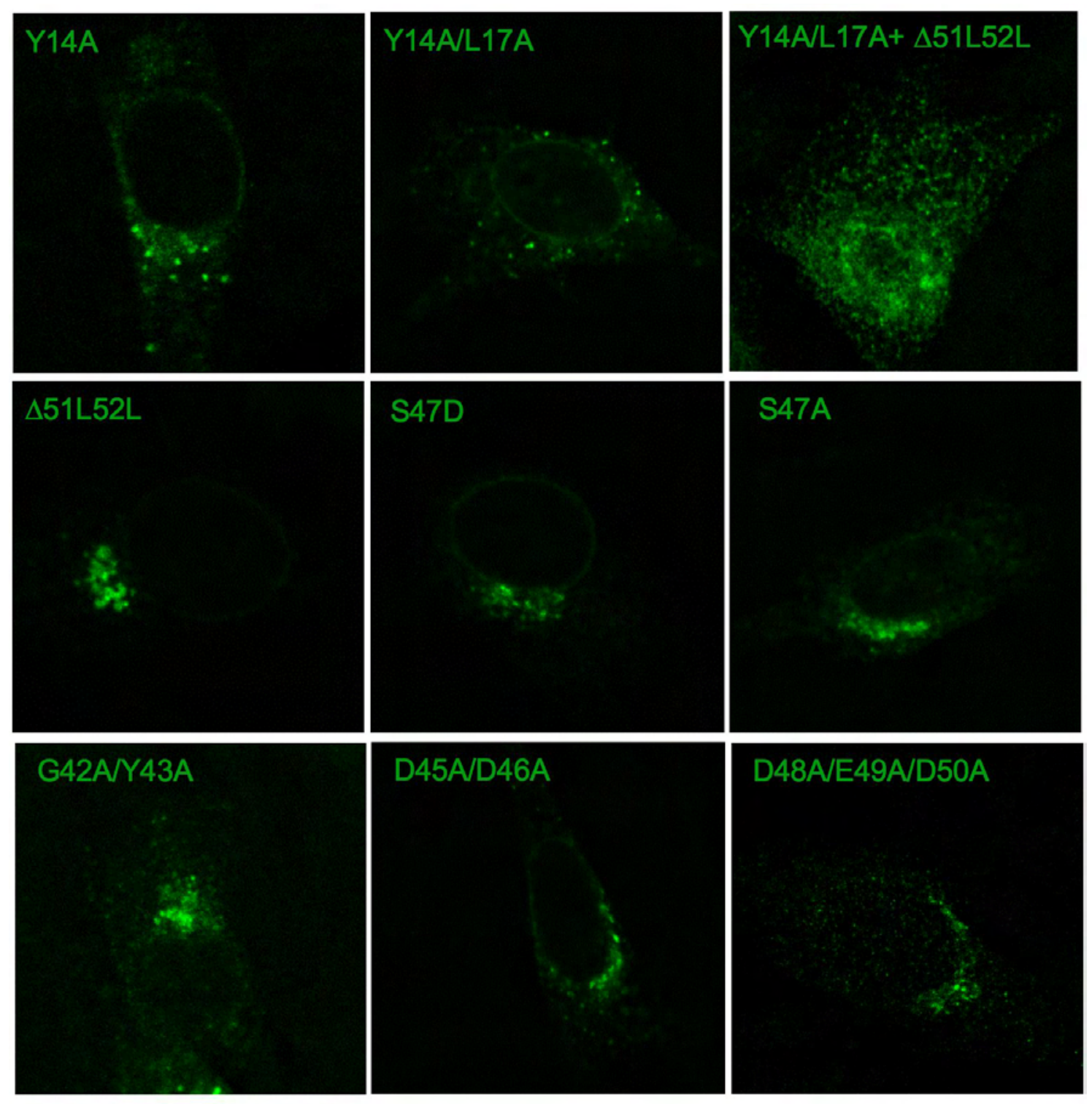

Figure 3.12: Steady state distribution of IL2R-Sor CD mutants in $\sigma 1 \mathrm{~B}-/$ - cells. IL2R-Sor $\mathrm{CD}$ mutants indicated in their respective images were transiently expressed in $\sigma 1 \mathrm{~B}$ deficient cells and the cells were fixed, permeabilized and incubated with primary antibodies against IL2R and then decorated with secondary antibodies conjugated with Alexa488. 


\subsubsection{Analysis of sortilin in adipocytes isolated from $\sigma 1 \mathrm{~B}$ 'knock- out' mouse}

Sortilin is highly expressed in adipocytes and is a major constituent of Glut4 vesicles, which are an intracellular pool of Glut4 glucose transporters released to the plasma membrane upon insulin stimulation. Adipose tissues express $\sigma 1 \mathrm{~A}$ and $\sigma 1 \mathrm{~B} 1 / \mathrm{B} 3$ but no $\sigma 1 \mathrm{~B} 2$ or $\sigma 1 \mathrm{C}$ (Riel, 2004). Therefore we analyzed sortilin expression levels in adipocytes isolated from control and $\sigma 1 \mathrm{~B}$-deficient mice by western blot (Fig. 3.13)

Two animals of each genotype were tested. The epididymal fat pads were isolated from the wild-type (wt) and $\sigma 1 \mathrm{~B}$ 'knock-out' mice and were digested with crude collagenase to disrupt the tissues. The adipocytes, which float in the top of the supernatant, were isolated from other tissues by low speed centrifugation. The isolated adipocytes were washed in homogenization buffer and homogenized using a teflon pestle homogenizer. This whole cell lysate was centrifuged to get rid of the nuclei and the post-nuclear supernatant was used for subsequent analysis. $100 \mathrm{\sigma g}$ of total protein from each sample were separated by 10\% SDS-PAGE, blotted onto PVDF membrane and decorated with antibody against sortilin (Fig. 3.13 A). The signals of sortilin were quantified by $2 \mathrm{D}$ densitometry and normalized to that of Hsc70. The resulting values corresponding to each genotype were averaged and the ratio of difference in expression levels of sortilin in $\sigma 1 \mathrm{~B}$-deficient tissues compared to wt is presented in the plot (Fig. $3.13 \mathrm{~B}$ ).

Both $\sigma 1 \mathrm{~B}$-deficient tissues showed a four fold increase in sortilin expression levels, after normalization of the signals against Hsc70 signals serving as control. These data indicate that the sortilin- AP-1 interactions are indeed important for sortilin trafficking in adipocytes. An increase in the amount of sortilin could be caused by a block in export out of one compartment, which would also block sortilin transport to lysosomes. However it could also be caused by an enhanced transcription/translation rate, induced by trapping of sortilin in one compartment or by enhanced degradation due to missorting to lysosomes. Further experiments with primary cell cultures will enable us to answer these questions. 
(A)

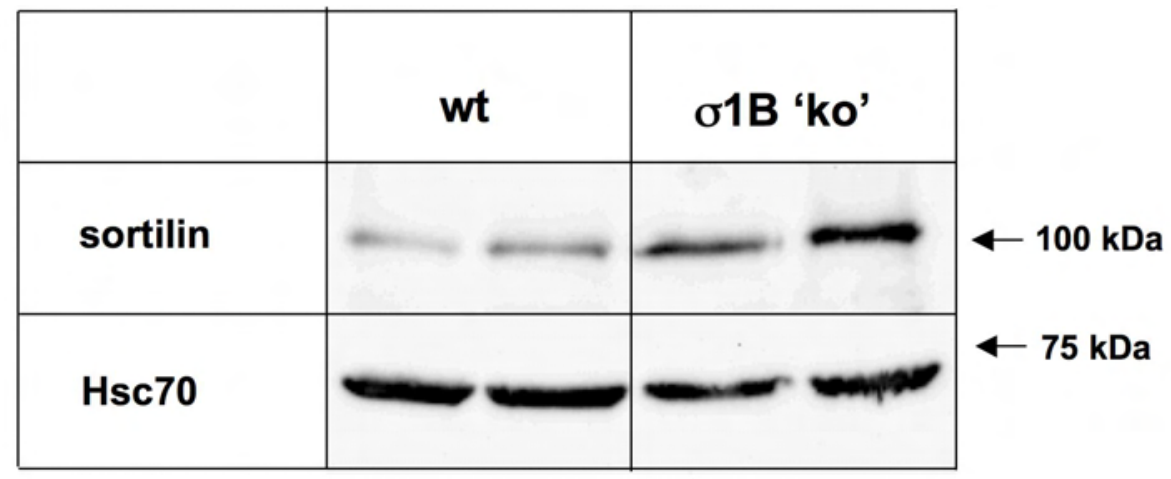

(B)

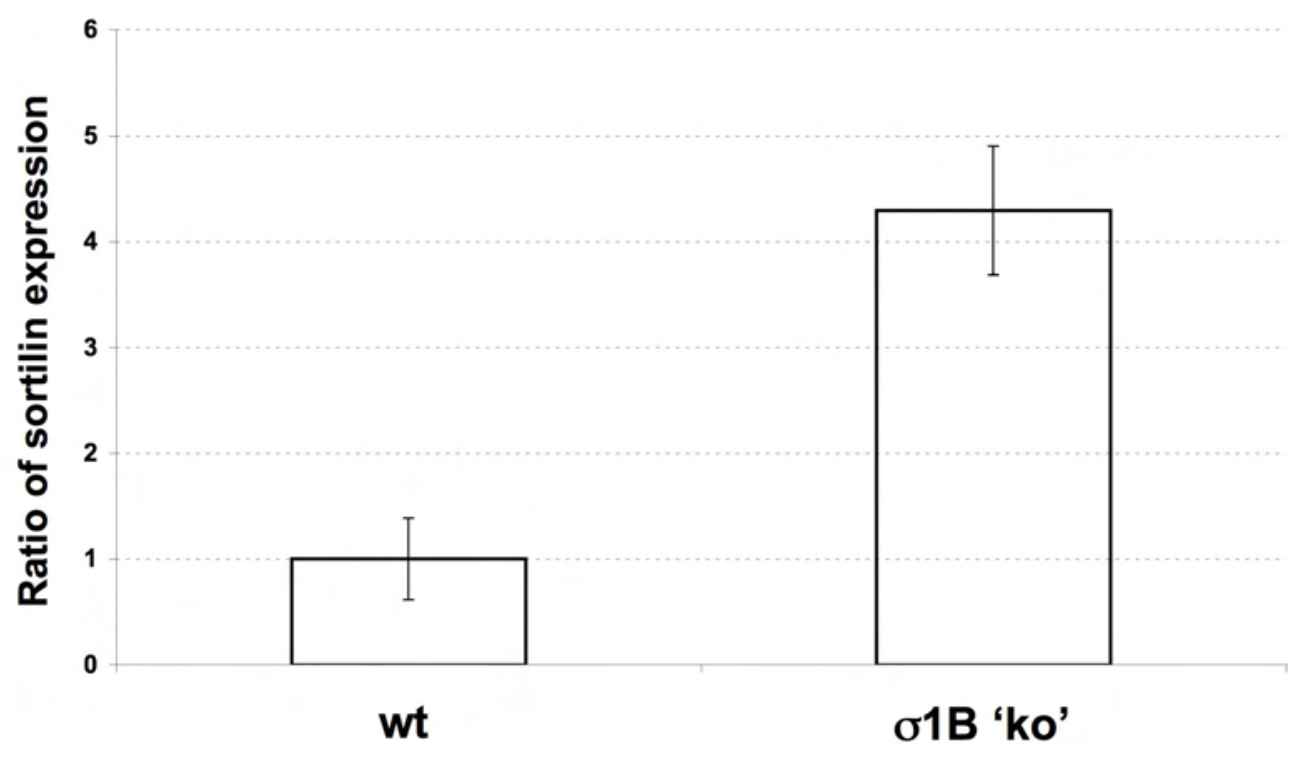

Figure 3.13: Analysis of sortilin in adipocytes isolated from $\sigma 1 \mathrm{~B}$ 'knock-out' mouse. (A) The whole cell lysate of adipocytes isolated from two wild-type (wt) and $\sigma 1 \mathrm{~B}$ 'knockout' mice were separated by $10 \%$ SDS-PAGE, blotted onto PVDF membrane and decorated with antibody against sortilin. Hsc70 served as a loading control. The relative position of the molecular weight marker is indicated. (B) The signals of sortilin were quantified by 2D densitometry and normalized to that of $\mathrm{Hsc70}$ and the ratio of difference in expression levels of sortilin in $\sigma 1 \mathrm{~B}$-deficient tissues compared to wt is represented. The error bars indicate the maximum deviation of values between samples of each genotype. 


\subsection{Specificity of $\gamma 1 / \sigma 1$ adaptin interactions}

Protein sorting by the heterotetrameric adaptor-protein complexes is mediated by the specificity of sorting motif recognition by these complexes as well as by their compartment specific membrane binding, which involves binding to specific phosphotidyl-inositol phosphates. In addition, a large number of proteins bind to the 'ear'-domains of $\gamma 1$ and $\alpha$-adaptin of AP-2. The function of these proteins is not understood, however the binding of some of these interferes with AP-membrane binding and thus protein sorting. An additional intra-molecular interaction of an 'ear'-domain was observed with the $\delta$-adaptin of the AP-3 complex. The $\delta$-adaptin 'ear'-domain binds to the $\sigma 3$-adaptin, interfering with membrane-binding of AP3 , presumably by sterically blocking ARF- 1 binding to the $\delta$-adaptin core domain (Lefrancois et al., 2004).

The $\sigma 1 \mathrm{~B} 3$-adaptin also contains a $\gamma 1$-adaptin 'ear' binding motif of the sequence 146EEWGGL151 (see Fig. 3.14 A). We therefore tested for $\gamma 1$-'ear' binding to $\sigma 1$ adaptins in the $\mathrm{Y} 2 \mathrm{H}$ system. $\gamma 1$-'ear' binding to rabaptin- $5 \alpha$ served as positive control (Fig.3.14). $\gamma$ 1-'ear' was constructed as a fusion of Gal4 activation domain (prey). Only the C-terminal tail fragments of $\sigma 1$-adaptins (aa 70-156), were chosen for the study, because the homology between the $\sigma 1$-adaptin isoforms was the least in this region. Also, the highly identical $\mathrm{N}$-terminal part is engaged in complex formation. The C-terminal tail fragments of $\sigma 1$-adaptins and rabaptin-5 $\alpha$ were constructed as a fusion of Gal4 DNA binding domain (bait). Both the bait and prey constructs were co-transformed into the yeast strain and allowed to grow in reporter gene selection plates as shown (Fig. 3.14 B). The results were recorded after allowing to grow for 3 to 5 days.

Surprisingly, only $\sigma 1 \mathrm{~A}$ bound to $\gamma 1$-'ear', but very weakly and via a different yet unidentified motif. But, $\sigma 1 \mathrm{~B} 3$ did not show any interaction. Therefore we speculated, that the neighbouring residues of the motif in $\sigma 1 \mathrm{~B} 3$-adaptin prevented the interaction. Indeed, deleting the preceding acidic residue E146 enabled $\gamma 1$-'ear' and $\sigma 1 \mathrm{~B} 3$ binding. Also, the mutation L151F increased the affinity of the motif so strong, that the negative effect of E146 was suppressed. This could indicate that the negative selection altered the motif to prevent interaction with the $\gamma 1$-'ear'-domain.

These data open the possibility that AP- $1 / \sigma 1 \mathrm{~A}$ and AP- $1 / \sigma 1 \mathrm{~B}$ membrane binding or transport vesicle formation are regulated in different ways enabling specific sorting functions of these AP-1 sub-complexes.

Since no cell line is available, which expresses just one $\sigma 1$-adaptin, this result could not be verified in vivo. However, cells from tissue of the $\sigma 1 \mathrm{~B}$ 'knock-out' mouse should allow us to perform these experiments. 
(A)

\begin{tabular}{|c|c|}
\hline NECAP1/2 & D/EWGx F/W \\
\hline $\begin{array}{l}\text { Rabaptin5 } \alpha \\
\gamma \text {-synergin }\end{array}$ & D/E F xx L/F/W/M \\
\hline б1B-3 & ${ }^{143}$ SQNEEWGGLSEDIL ${ }^{156}$ \\
\hline$\Delta \mathrm{E} 146$ & ........EWGGL......... \\
\hline G149V & ........EEWVGL ......... \\
\hline L151F & $\ldots \ldots \ldots E W G G F \ldots \ldots \ldots$ \\
\hline
\end{tabular}

(B)

$\gamma 1$ ear-hinge

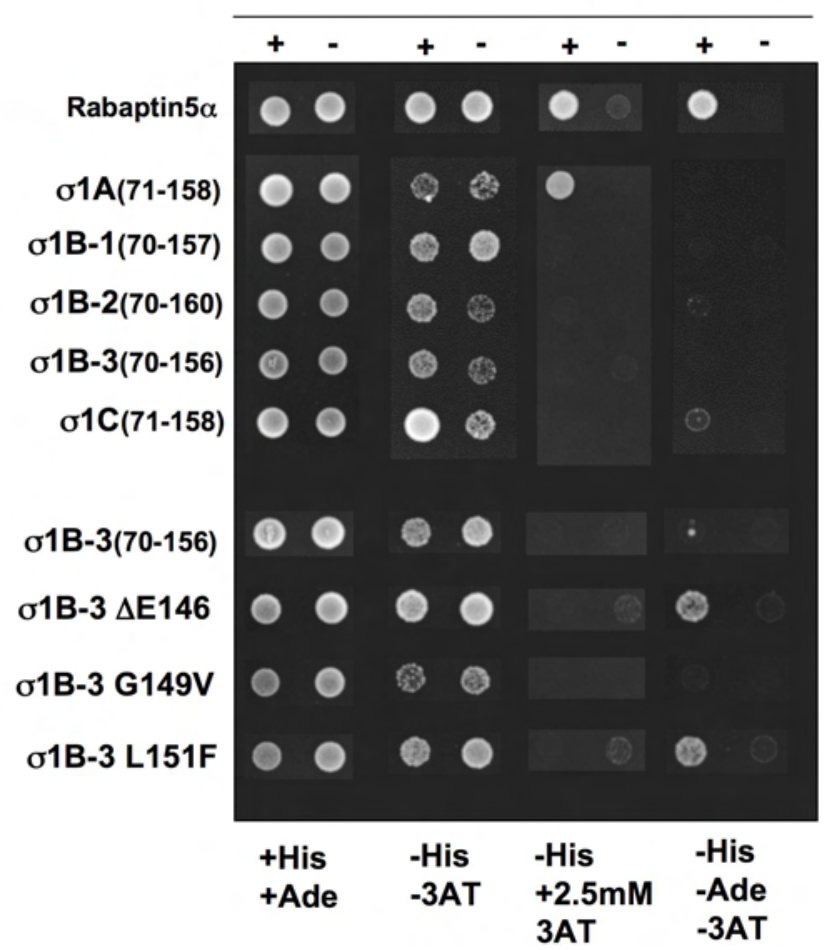

Figure 3.14: Analysis of interaction between $\gamma$ 1-'ear'-hinge domain and C-terminal tail of $\sigma 1$-adaptins. A) $\gamma 1$-'ear' binding motifs present in respective proteins. Putative $\gamma 1$-'ear' binding motif present in $\sigma 1 \mathrm{~B}-3$ and the mutants constructed for this study are depicted in bold. (B) $\mathrm{Y} 2 \mathrm{H}$ analysis of interaction between $\gamma 1$-'ear'-hinge (as fusion of Gal4 DNA binding domain) and C-terminal tail of $\sigma 1$ isoforms and of $\sigma 1 \mathrm{~B}-3$ mutants (as fusion of Gal4 activation domain). Rabaptin $5 \alpha$ served as a positive control. The interaction was assessed based on the growth of yeast in reporter gene selection plates as shown. 


\subsection{A novel role for the $\mathrm{N}$-terminal domain of $\mu 1 \mathrm{~A}$ in regulation of AP-1 function}

\subsubsection{Insights from previous studies on $\mu 2 / \mu 1$-chimeric AP-1 complex}

Previous study from our group provided evidence that the N-terminal seventy aminoacids of $\mu 1 \mathrm{~A}$ has a role in maintaining the fidelity of the sorting function of AP-1 complex ((Medigeshi Ramarao, 2003), Medegeshi Guruprasad and P. Schu, unpublished). In this study, domains of $\mu 1 \mathrm{~A}$ were replaced by those of $\mu 2$ to identify functions of $\mu 1 \mathrm{~A}$ domains.

(A)

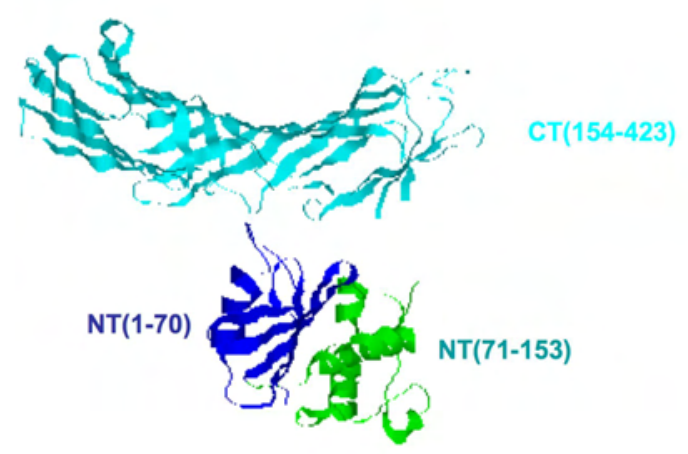

(B)

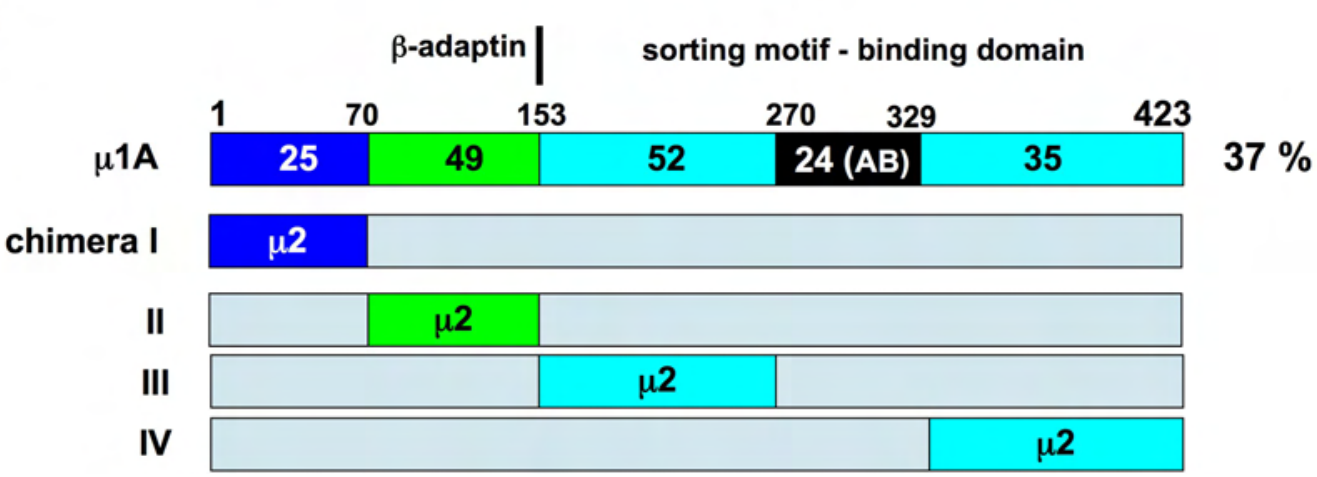

Figure 3.15: Design of $\mu 2 / \mu 1$ chimeras. (A) Structural model of $\mu$-adaptins colored for different domains used for designing chimeras. (B) Schematic representation of homology between $\mu 1 \mathrm{~A}$ and $\mu 2$-adaptins and strategy of chimera design. Numbers above the bar indicate the aminoacid number; within the bar indicate percent homology between domains as represented by colors in $(A)$; overall homology $37 \%$. $A B$ indicates antibody binding region in $\mu 1 \mathrm{~A}$, against which the antiserum for $\mu 1 \mathrm{~A}$ is directed. Chimera $(\mathrm{I}, \mathrm{II}, \mathrm{III}, \mathrm{IV})$ represent the different $\mu 1 \mathrm{~A}$ chimeras that were constructed and the colors indicate the position of replacement with corresponding $\mu 2$ domains represented in $(A)$. 
When expressed in $\mu 1 \mathrm{~A}$-deficient cells, only chimera I was able to incorporate into a chimeric heterotetrameric adaptor complex, designated AP- $1^{*}$, with functional membrane binding properties as evidenced by the restoration of the steady-state localization of $\gamma 1$ adaptin, clathrin and MPRs to the peri-nuclear area. This was surprising, because in this $\mu 2 / \mu 1 \mathrm{~A}$ chimera, the $\mathrm{N}$-terminal 70 amino acid residues of $\mu 1 \mathrm{~A}$ were replaced with those of $\mu 2$, the region that showed the least sequence homology (Fig. 3.15). We compared the structure of $\mu 1 \mathrm{~A}$ and $\mu 2 \mathrm{~N}$-terminal

(A)

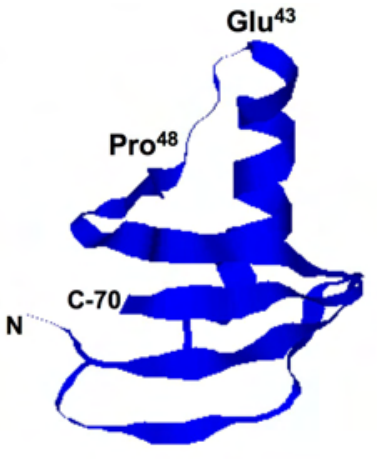

$\mu 1 \mathrm{~A}(4.0 \mathrm{~A})$

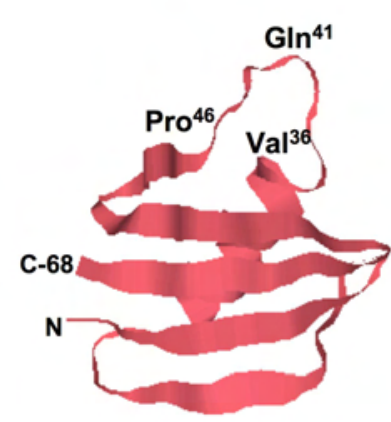

$\mu 2(2.59 \AA)$

(B)

$$
\begin{aligned}
& \text { } 42 \text { MIGG-LFIYNHKGEVLISRVYRDDIGRNAVDAFRVNVIHARQQVR-SPVTNIARTSFFHVKRSNIWLAAV } \\
& \mu 1 \mathrm{~A} \text { MSASAVYVLDLKGKVLICRNYRGDVDMSEVEHFMPILMEKEEEGMSPILAHGGVRFMWIKHNNLYLVAT }
\end{aligned}
$$

Figure 3.16: Comparison of $\mathrm{N}$-terminal seventy amino acid domains of $\mu 1 \mathrm{~A}$ and $\mu 2$ adaptins. (A) Structural similarity of $\mu 1 \mathrm{~A}$ - and $\mu 2-\mathrm{N}$-terminal seventy amino acid domains. (B) Primary sequence comparison of the $\mathrm{N}$-terminal seventy amino acids of $\mu 2$ and $\mu 1 \mathrm{~A}$-adaptins. Identical residues are indicated in bold.

domains, containing the first seventy amino acids, based on the recently solved X-ray crystallographic structures of AP-1 and AP-2 ((Heldwein et al., 2004) and (Collins et al., 2002) respectively). It revealed that, although the primary sequence had the least homology, the tertiary structure is highly conserved. This high degree of structural conservation is responsible for the wt level expression of AP-1* Fig. 3.16).

However, when MPR dependent cathepsin D sorting was followed, AP- $1^{*}$ expressing cells showed an intermediate mis-sorting phenotype compared to control and $\mu 1 \mathrm{~A}$-deficient cells. In the presence of $5 \mathrm{mM}$ mannose 6-phosphate, $40 \%$ of the Golgi precursor form of cathepsin D was mis-sorted into secretions in AP$1^{*}$ expressing cells, compared to $61 \%$ in $\mu 1 \mathrm{~A}$-deficient and $10-15 \%$ in control cells, demonstrating an impaired sorting function of AP- $1^{*}$ leading to only a partial complementation of AP-1-deficiency. Time dependent redistribution of AP-1 
and AP- $1^{*}$ between membrane and the cytoplasm were followed by addition of Brefeldin A, a drug that interferes with ARF-1 mediated AP-1 membrane recruitment and thereby leads to the redistribution of membrane-bound AP-1 to cytoplasm. These experiments revealed that AP- ${ }^{*}$ redistribution was slowed-down compared to AP-1 indicating that its membrane-cytoplasm recycling dynamics were disturbed. Based on these preliminary results it was proposed that, the N-terminal seventy aminoacids of $\mu 1 \mathrm{~A}$ play a role in the regulation of cytoplasm-membrane recycling of AP-1, presumably by binding to and recruiting yet unknown protein(s) and thus regulate AP-1 sorting function.

In the present study, using AP- $1^{*}$ expressing cells as a model system, we attempted to further characterize and define the role of N-terminal seventy amino acids of $\mu 1 \mathrm{~A}$-adaptin in the regulation of AP-1 sorting function.

\subsubsection{Intracellular membrane morphology in AP-1* expressing cells}

Since AP-1* expressing cells showed abnormality in lysosomal delivery of cathepsin $\mathrm{D}$, we were interested in looking for any alterations in membrane morphology in these cells compared to control ( $\mu 1 \mathrm{~A}-/-:: \mu 1 \mathrm{~A})$ and AP-1-deficient ( $\mu 1 \mathrm{~A}-/-)$ cells by electron microscopy. As shown in (Fig. 3.17 B), AP-1-deficient cells exhibited large membranous structures in the cytoplasm, compared to control cells (Fig. 3.17 A). Interestingly, AP-1* expressing cells also contained these large structures as seen in AP-1-deficient cells, (Fig.3.17 C) indicating that AP-1* expression in $\mu 1 \mathrm{~A}$-deficient cells could not rescue the phenotype completely, consistent with the intermediate phenotype observed in cathepsin D sorting (Medigeshi Ramarao, 2003).

When probed for clathrin-heavy-chain and $\gamma 1$-adaptin by immunogold-labelling, these structures exhibited a weak labelling (Fig. 3.17 D,E). To reveal the identity of these large membranous structures, the cells were allowed to endocytose BSAgold for 10 and 30 minutes and were also labelled for LAMP-1, a marker for late endosomes/lysosomes. As shown in fig. 3.16 F, after 10 minutes, those enlarged organelles were loaded with BSA-gold indicating that they are connected to the endocytic pathway. A low level of LAMP-1 labelling in these organelles, consistent with kinetic characterization (Griffiths et al., 1989), indicate these organelles as early endosomes. After 30 minutes, endocytosed BSA-gold were also found in late endosomes, characterized by presence of internal vesicles and a high density of LAMP-1 labelling (Fig. 3.17 G), demonstrating vesicle transport between these organelles. 

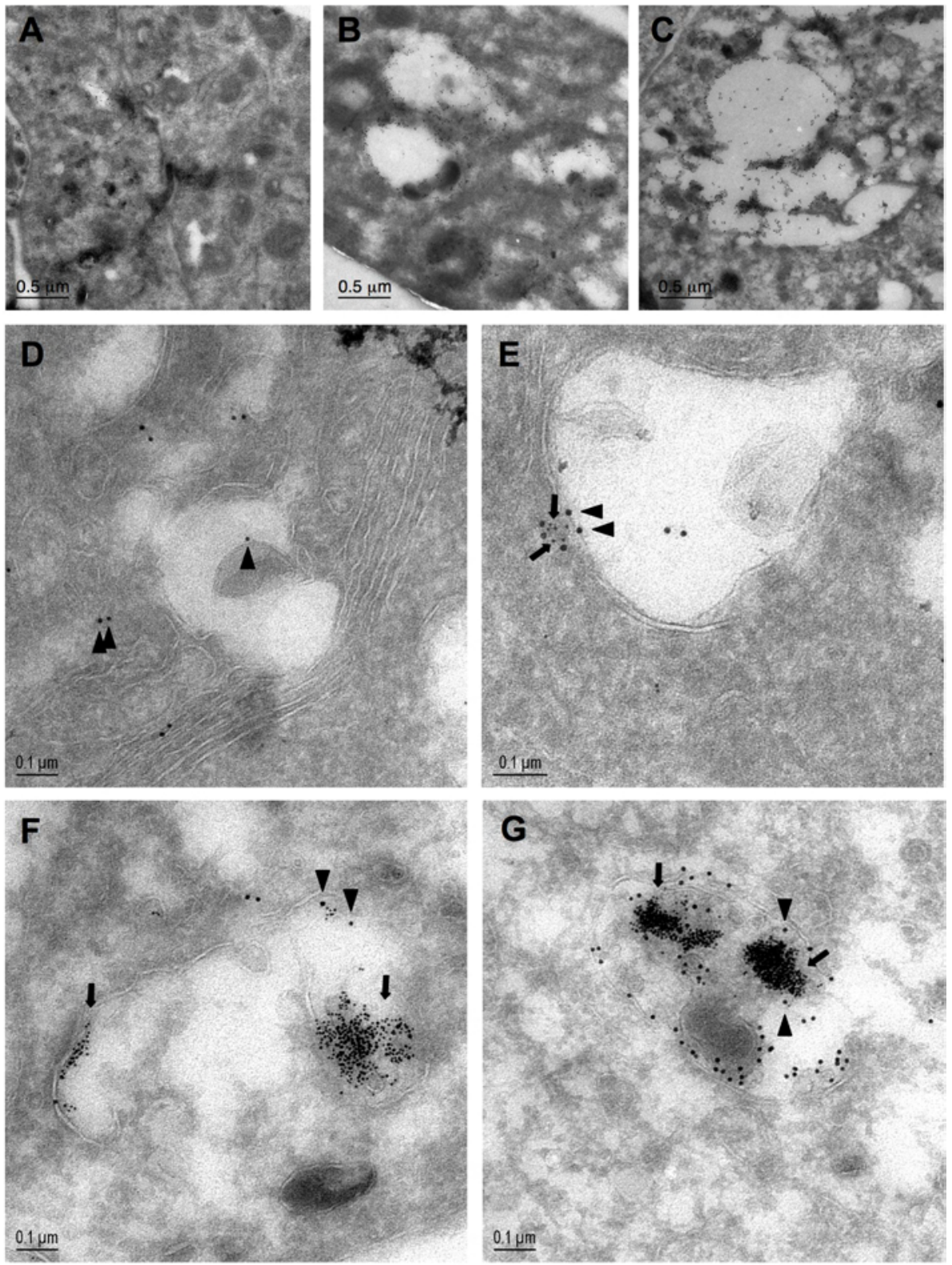

Figure 3.17: Electron microscopy of AP-1, AP-1-deficient ( $\mu 1 \mathrm{~A}-/-)$ and AP-1* expressing cells. Overview of membrane morphology in (A) control cells, (B) AP-1-deficient cells and (C) AP-1* expressing cells. Large organelles in AP-1* expressing cells (D, E). Arrow tips point to clathrin labelling ( $10 \mathrm{~nm}$ gold) and arrows point to $\gamma 1$-adaptin labelling ( $5 \mathrm{~nm}$ gold). Organelles labelled in AP-1* cells after 10 min (F) and 30 min (G), BSA-gold endocytosis. In (F) and (G), arrow tips point to LAMP-1 labelling (10 $\mathrm{nm}$ gold) and arrows to BSA-gold ( $5 \mathrm{~nm}$ gold). 


\subsubsection{Analysis of AP-1* membrane recycling at the TGN}

It has been shown that membrane bound clathrin and adaptors are in dynamic equilibrium with cytoplasmic pool of free clathrin and adaptors, through continuous and rapid exchange between the membrane bound and soluble state and interestingly, that adaptors recycling also occurs independent of vesicle budding (Wu et al., 2001). In a subsequent study, $\gamma 1$ labelled with EGFP was used to study the recycling kinetics of AP-1 at the TGN, indicating that the presence of EGFP does not affect the function of $\gamma 1$-adaptin (Wu et al., 2003). Since, we had already observed that in the presence of BFA, the redistribution of AP- ${ }^{*}$ was slower than AP-1 (Medigeshi Ramarao, 2003), we analyzed the membrane recycling dynamics of AP-1 and AP- ${ }^{*}$ in cells transiently expressing EGFP- $\gamma 1$, using fluorescence recovery after photobleaching (FRAP). This method allows us to bleach a region of interest (ROI) and measure the fluorescence recovery in that ROI, which is a direct measure of the exchange dynamics of bleached membrane-bound AP-1 with unbleached cytoplasmic ones. As shown in Fig. 3.18, the recovery of fluorescence after photobleaching for 10 $s$ was measured in the central TGN region at room temperature either in normal buffer conditions or after treating the cells with $0.2 \mathrm{M}$ sucrose for 30 minutes. The fluorescence recovery half-time $\left(\mathrm{t}_{1 / 2}\right)$ in seconds $(\mathrm{s})$, was calculated from a plot of fluorescence values against time (in s) as shown in Fig. 3.19.

The kinetics of EGFP- $\gamma 1$-adaptin FRAP is depicted in the Fig. 3.19 which is an average of indicated total number(n) of experiments under normal buffer conditions. AP- $1^{*}$ showed an almost $50 \%$ reduction in recycling kinetics compared to AP-1, with a $t_{1 / 2}$ of $17 \pm 2 \mathrm{~s}$ and $11 \pm 2 \mathrm{~s}$, respectively. However, both AP- $1^{*}$ and AP-1 exhibited similar average time-course of recovery $\left(\mathrm{A}_{\text {recov }}\right)$ values; $0.93 \pm 0.05$ for AP-1 and $0.83 \pm 0.07$ for $\mathrm{AP}-1^{*}$, ie., $\sim 85-90 \%$ of recovery at the measured maximum time point. This indicates that membrane-bound AP-1* exchanges with the cytoplasmic pool but with a reduced rate.

It has been reported that hypertonic sucrose induces the formation of empty clathrin cage baskets and the exchange of clathrin is completely blocked in the presence of hypertonic sucrose or upon $\mathrm{K}^{+}$depletion, whereas AP-1 and GGA still exchange, but with reduced rates ( $\mathrm{Wu}$ et al., 2003). We were interested to see the effect of clathrin exchange on AP-1*, as it can be argued that the observed reduction in AP- $1^{*}$ recycling could be an indirect consequence of defective clathrin uncoating process. We performed EGFP- $\gamma 1$ FRAP measurements in AP- ${ }^{*}$ and AP-1 expressing cells, wherein the exchange of clathrin at coated pits were blocked by prior incubation of cells in hypertonic sucrose (0.2 M) for 30 minutes (Fig. 3.18 C).

As shown in fig. 3.19 (B), even after blocking clathrin exchange and thereby 


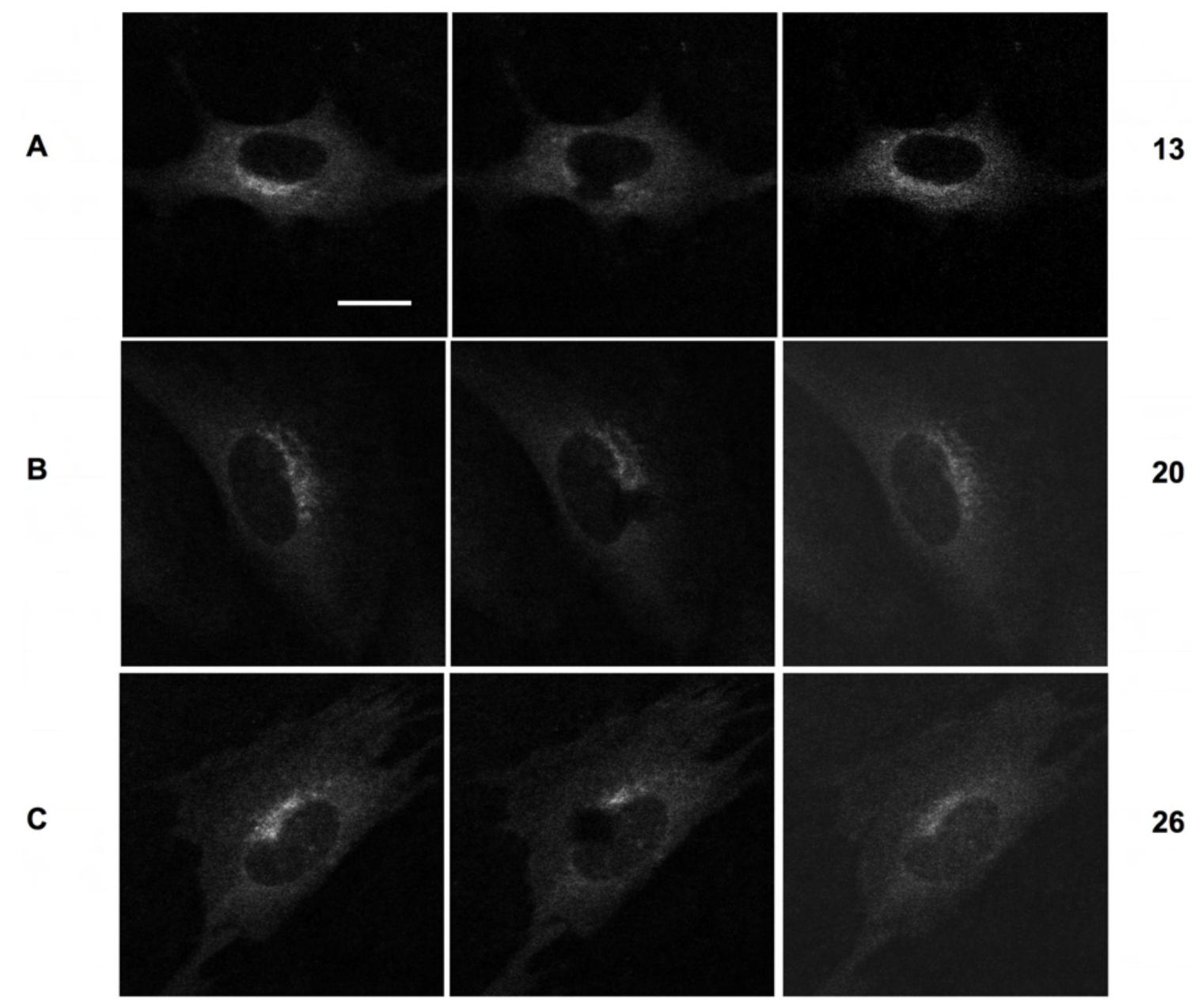

Figure 3.18: Fluorescence recovery after photobleaching in AP-1 and AP-1* expressing cells . EGFP- $\gamma 1$ expressing control cells (A) and AP-1* cells (B) were subjected to photobleaching at the central TGN area, for $10 \mathrm{~s}$ and the fluorescence recovery was monitored under normal buffer conditions at $37^{\circ} \mathrm{C}$. Panel $\mathrm{C}$ shows FRAP analysis in AP- $1^{*}$ cells treated with $0.2 \mathrm{M}$ sucrose for $30 \mathrm{~min}$. Numbers correspond to the observed fluorescence recovery half-time $\left(\mathrm{t}_{1 / 2}\right)$ in seconds.

vesicle budding, recovery of fluorescence for AP-1 was observed but at a slower rate with a $t_{1 / 2}$ of $20 \pm 6 \mathrm{~s}$, in agreement with the reported study (Wu et al., 2003). Also for AP-1*, we observed a reduction in the fluorescence recovery with a $t_{1 / 2}$ of $32 \pm 6 \mathrm{~s}$ in a pattern as observed under normal conditions. Moreover, AP-1 exhibited similar time-course of recovery, $\sim 85 \%$ as observed under normal conditions (fig. 3.19 B). But, AP- $1^{*}$ exhibited a remarkable reduction in the average time-course of recovery, $\sim 60 \%$, when clathrin exchange is blocked, indicating that when vesicle budding is blocked, the fraction of AP- $1^{*}$ binds to the membrane firmly, presumably at non-productive sites and exchanges less with the cytoplasmic pool. These results indicate, that the observed reduced recycling of $\mathrm{AP}-1^{*}$, is a direct consequence of 
(A)

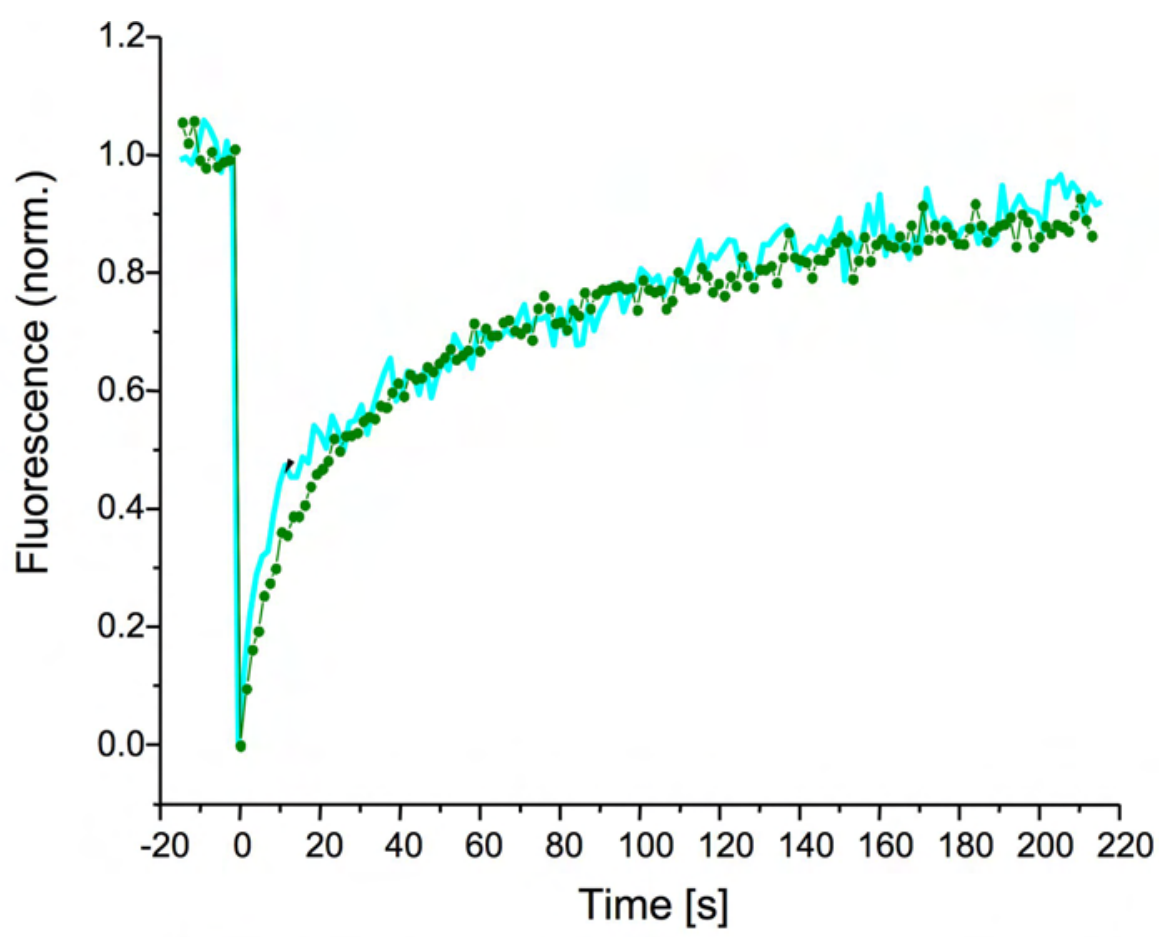

$$
\begin{aligned}
\rightarrow \mathrm{AP}-1^{*} \tau_{1 / 2} & =17 \pm 2 \mathrm{~s} \mathrm{~A}_{\text {recov }}=0.93 \pm 0.05, \mathrm{n}=8 \\
-\mathrm{AP}-1 \tau_{1 / 2} & =11 \pm 2 \mathrm{~s} \mathrm{~A}_{\text {recov }}=0.83 \pm 0.07, \mathrm{n}=6
\end{aligned}
$$

(B)

\section{Sucrose $0.2 \mathrm{M} 30 \mathrm{~min}$ :}

$$
\begin{array}{ll}
\mathrm{AP}-1^{*} \tau_{1 / 2}=32 \pm 4 \mathrm{~s} & \mathrm{~A}_{\text {recov }}=0.62 \pm 0.14, \mathrm{n}=9 \\
\mathrm{AP}-1 \tau_{1 / 2}=20 \pm 6 \mathrm{~s} & \mathrm{~A}_{\text {recov }}=0.86 \pm 0.06, \mathrm{n}=6
\end{array}
$$

Figure 3.19: Kinetics of fluorescence recovery after photobleaching of AP-1 and AP$1^{*}$. (A) The plot depicts the kinetcs of fluorescence recovery of EGFP-AP-1 and EGFP-AP-1* measured at indicated time points (seconds) after photobleaching for $10 \mathrm{~s}$ under normal buffer conditions and at $37^{\circ} \mathrm{C}$ in control and $\mathrm{AP}-1^{*}$ expressing cells. $\mathrm{A}_{\text {recov }}$ indicates the average time-course of complete recovery. (B) Statistics of fluorescence recovery after photobleaching for EGFP-AP-1 and EGFP-AP-1* measured after hypertonic sucrose treatment. $\mathrm{n}$ corresponds to number of individual experiments. 
altered dynamics in membrane binding and exchange with free cytoplasmic AP-1*.

\subsubsection{Analysis of known determinants that regulate AP-1/AP-1* membrane binding}

The data from previous section, clearly demonstrate that the N-terminal seventy amino acids of $\mu 1 \mathrm{~A}$ play a role in AP- 1 membrane binding dynamics. As a next step, we were interested to probe on the mechanism by which the N-terminus of $\mu 1 \mathrm{~A}$ operates to execute its regulatory function.

The crystal structure of AP- 1 reveals that the N-terminal seventy amino acid domain of $\mu 1 \mathrm{~A}$ (colored blue in Fig. 3.20) are not in contact with the membrane, excluding direct $\mu$-lipid interactions. However, it is accessible from the cytoplasmic face of the heterotetrameric adaptor complex (Fig. 3.20).

At the membrane face of the complex, the C-terminal sorting motif binding domain of $\mu 1 \mathrm{~A}$-adaptin shields the $\mu 1 \mathrm{~A} \mathrm{~N}$-terminus. However, in the phosphorylationinduced activated state, the sorting-motif binding domain has to flip out of the core complex in order to be able to interact with the cytoplasmic tails of transmembrane proteins, which again exposes the $\mathrm{N}$-terminal domain.

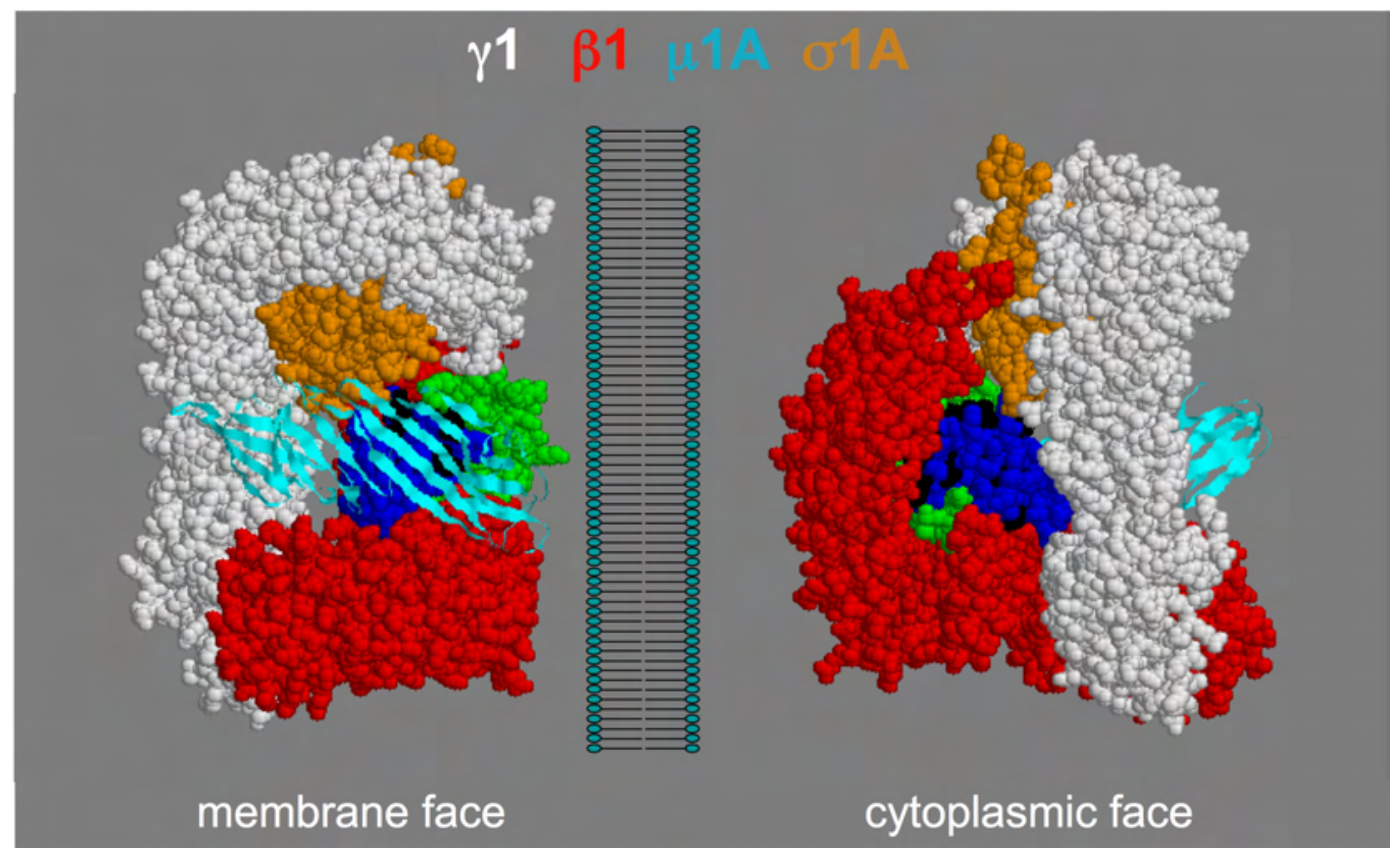

Figure 3.20: Position of $\mathrm{N}$-terminal seventy amino acids of $\mu 1 \mathrm{~A}$ in AP-1 complex. The subunits are represented by their depicted color. The $\mathrm{N}$-terminal seventy amino acids of $\mu 1 \mathrm{~A}$ is shown in blue; $\mathrm{C}$-terminal domain of $\mu 1 \mathrm{~A}$ in cyan.

Several studies over the past years have identified cytosolic and membrane 
bound factors to play a role in regulating the recruitment of AP-1 onto membranes. The N-terminal seventy amino acids of $\mu 1 \mathrm{~A}$ could recruit proteins directly or stabilize already recruited proteins that are known to be involved in AP-1 membrane binding. It is also possible that, as yet unknown cytoplasmic accessory and regulatory proteins involved in AP-1 mediated protein transport can bind to the $\mu 1 \mathrm{~A}$ N-terminus and regulate AP-1 membrane binding.

\subsubsection{Influence of phosphorylation kinetics on AP-1* membrane binding}

To gain insight on the reduced membrane-cytoplasm recycling of AP- $1^{*}$, as observed in FRAP experiments, we were interested to look for the phosphorylation state of AP- $1^{*}$, as it has been shown that the membrane recruitment of AP-1 is regulated by phosphorylation and de-phosphorylation cycles (Ghosh and Kornfeld, 2003a); the $\beta 1$ subunit is phosphorylated in the cytoplasm, but will be dephosphorylated if AP-1 is bound to membrane, whereas $\mu 1 \mathrm{~A}$ is in the dephosphorylated state in the cytoplasm, but phosphorylated in the membrane bound state. Also it is known that phosphorylation of $\mu 1 \mathrm{~A}$ subunit induces high affinity binding to the cargo sorting motif facilitated by a conformational change in $\mu 1 \mathrm{~A}$ to an open conformation, as observed by X-ray crytallographic studies and sorting motif affinity analyses for AP2 (Collins et al., 2002). In the phosphorylation-induced open conformation, the hinge region of $\mu 1 \mathrm{~A}$, that connects the $\mathrm{N}$ - and C-terminal domains, is accessible for trypsin digestion in vitro. Therefore susceptibility to trypsin digestion is an indirect measure of the phosphorylation state of $\mu 1 \mathrm{~A}$ and we took advantage of this property to analyze AP- $1^{*}$.

Adaptor molecules were stripped off the membrane fractions prepared from control and AP-1* expressing cells and subjected to partial trypsin digestion by incubation at $37^{\circ} \mathrm{C}$ for $15 \mathrm{~min}$, with increasing concentrations of trypsin. After inhibition of trypsinization, the samples were boiled in 1X Laemlli buffer and loaded on a $10 \%$ SDS-PAGE and decorated with rabbit anti- $\mu 1$-adapin antibody, which recognizes both $\mu 1 \mathrm{~A}$ and Chimera I-adaptins. The signals were quantified and the percentage of undigested $\mu 1$-adaptins were plotted against trypsin concentration.

As shown in Fig. 3.21, $\mu 1 \mathrm{~A}$ exhibits a dramatic dose dependent tryptic degradation pattern when compared to Chimera I, indicating that Chimera I is less sensitive to tryptic digestion than $\mu 1 \mathrm{~A}$. At the highest concentration of trypsin used $(1.5$ $\mu \mathrm{g} / \mathrm{ml}), \sim 60 \%$ of Chimera I remains undigested compared to $\sim 30-40 \%$ of $\mu 1 \mathrm{~A}$, an approximately two-fold decrease in degradation, indicating that a larger fraction of Chimera $I$ is inaccessible for trypsin. This means that the membrane fraction of Chimera I is in an under-phosphorylated state. Two possibilities can be derived 


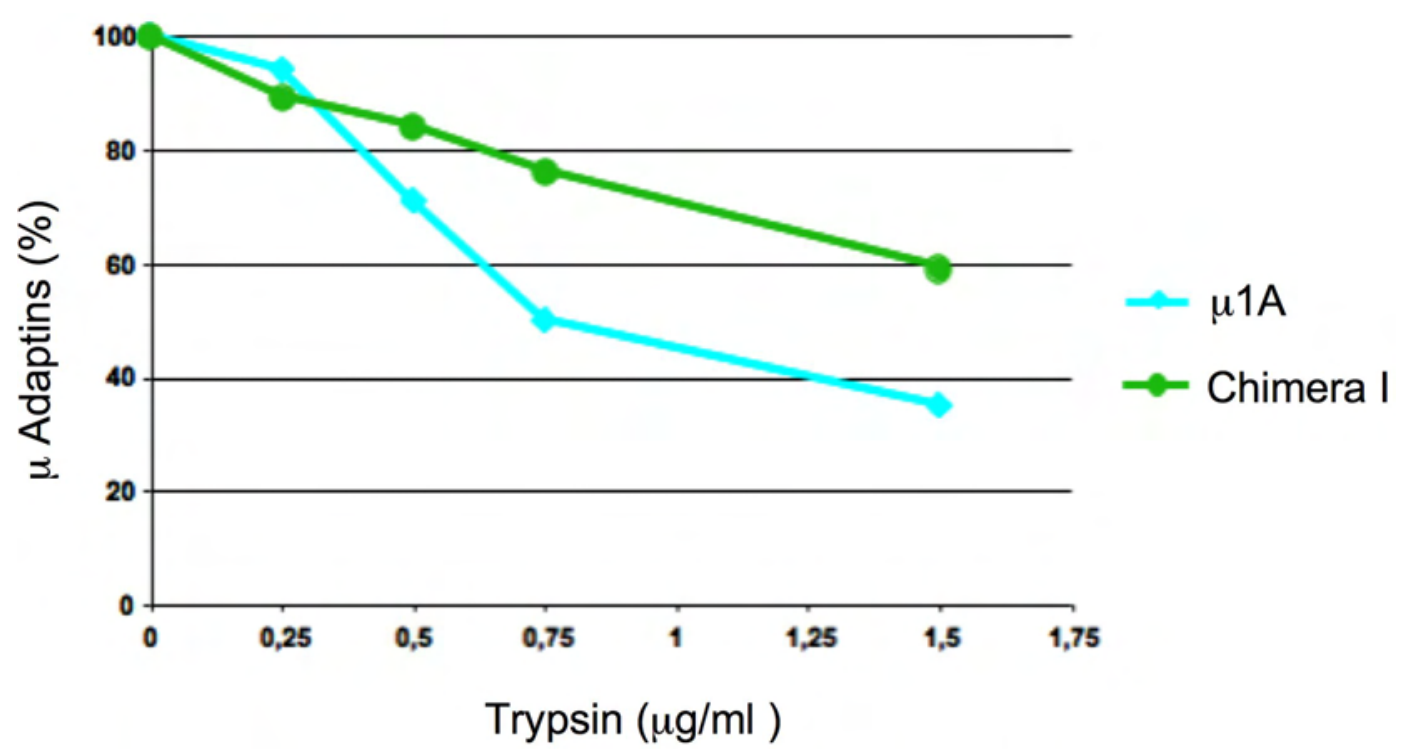

Figure 3.21: Dose dependent tryptic digestion of $\mu 1 \mathrm{~A}$ and Chimera I -adaptins. AP-1 and $\mathrm{AP}-1^{*}$ prepared by stripping the membrane fraction of control and AP-1* expressing cells, were subjected to partial trypsin digestion and analyzed by SDS-PAGE for the levels of undigested $\mu 1 \mathrm{~A}$ and Chimera I-adaptins using rabbit anti- $\mu 1$-adaptin antibody. The signals were quantified and plotted as a function of percent undigested adaptin against increasing concentrations of trypsin. The data presented is a representative of four independent experiments $(n=4)$.

for the state of under-phosphorylation; either due to increased de-phosphorylation or reduced phosphorylation combined with increased phosphorylation-independent membrane (or cargo) binding.

\subsubsection{2 $\mathrm{Y} 2 \mathrm{H}$ analysis of interaction between $\mathrm{N}$-terminal seventy amino acids of $\mu 1 \mathrm{~A}$ and candidate proteins}

By using $\mathrm{Y} 2 \mathrm{H}$ method, we analyzed the interaction of N-terminal seventy aminoacids of $\mu 1 \mathrm{~A}$ with candidate proteins viz., BIG2, cyclin $\mathrm{G}$ associated kinase (GAK)/ auxilin 2, the B-subunit of Protein phosphatase 2A (PP2A) and $\gamma$-BAR ( $\gamma 1$ adaptin Brefeldin A Resistance) which were shown to be involved in regulating the membrane binding of AP-1 (Fig. 3.22). BIG2 was identified as a guanine-nucleotide exchange factor(GEF) for ARF-1 operating at the TGN, which when overexpressed, blocked the Brefeldin A-induced redistribution of AP-1 from membranes, indicating that it is implicated in AP-1 membrane binding (Shinotsuka et al., 2002). The mechanism of BIG2 recruitment onto membranes is known to be independent of AP-1 or ARF-1.

$\gamma$-BAR is a recently identified peripheral membrane protein that was shown to interact directly with $\gamma$-appendage domain of AP- 1 and its overexpression was 

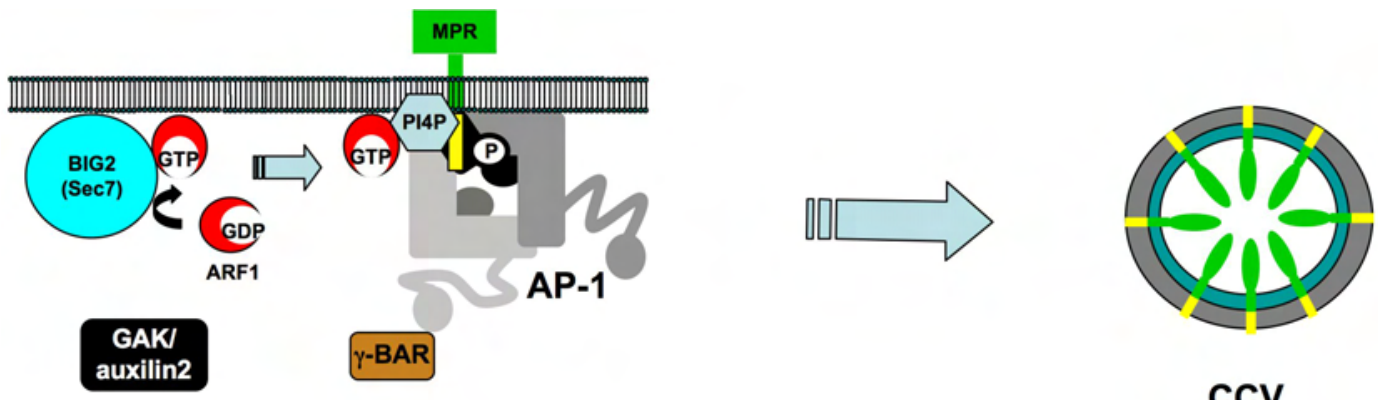

CCV

Figure 3.22: A model depicting the role of candidate proteins in AP-1 membrane recruitment and productive CCV formation.

shown to inhibit Brefeldin A-induced redistribution of AP-1 while its 'knock-down' directly reduced the membrane association of AP-1 (Neubrand et al., 2005). The precise mechanism of action of $\gamma$-BAR is not known. Since, our previous results showed that AP-1* exhibited a slow redistribution from the membrane to the cytoplasm upon Brefeldin A treatment, we chose BIG2 and $\gamma$-BAR as potential candidates to test for interaction with N-terminal seventy aminoacids of $\mu 1 \mathrm{~A}$, as both the proteins are known to be directly involved in the recruitment of AP-1 onto the membrane.

GAK was identified as a kinase, that is involved in activating hsc70-mediated clathrin uncoating and it was shown to interact with the $\gamma$-appendage domain of AP-1 complex (Umeda et al., 2000). Also, it was shown to be one of the major protein kinases associated with CCVs and the one that predominantly mediates CCVassociated kinase activity (Korolchuk and Banting, 2002).

PP2A $\beta$ was shown to play a role in the dephosphorylation of $\mu 1$ and $\beta 1$ subunits and its activity was shown to regulate the membrane association and dissociation cycles of AP-1 (Ghosh and Kornfeld, 2003a). PP2A is a complex of three subunitsA, B and C. Subunit A, acts as a scaffold for complex formation and subunit C is the catalytic subunit. We chose subunit B for our studies, because it is the regulatory subunit that provides substrate specificity for the phosphatase. We were interested in GAK and PP2A-B subunit, as to ascertain for their role in the altered phosphorylation state of AP- ${ }^{*}$.

The cDNAs encoding various domains of BIG2 (hBIG2), GAK and $\gamma$-BAR and the full length PP2A-B subunit were cloned into the prey vector(pACT2) and the Nterminal seventy amino acid domain of $\mu 1 \mathrm{~A}$ was cloned into the bait vector (pGBT9) and co-transformed into yeast strain AH109. The co-transformants were allowed to grow at $30^{\circ} \mathrm{C}$ in medium lacking histidine and adenine and results were recorded after 3 to 5 days. As shown in Fig. 3.23, none of the co-transformants showed 
(A)

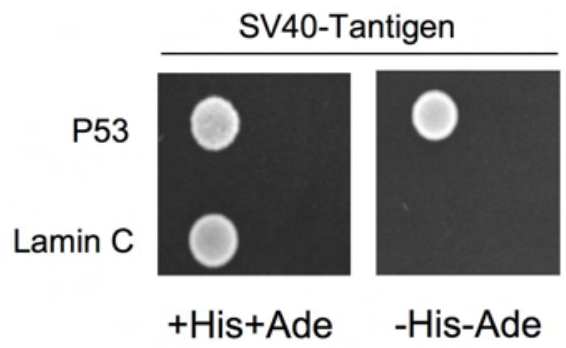

(C)

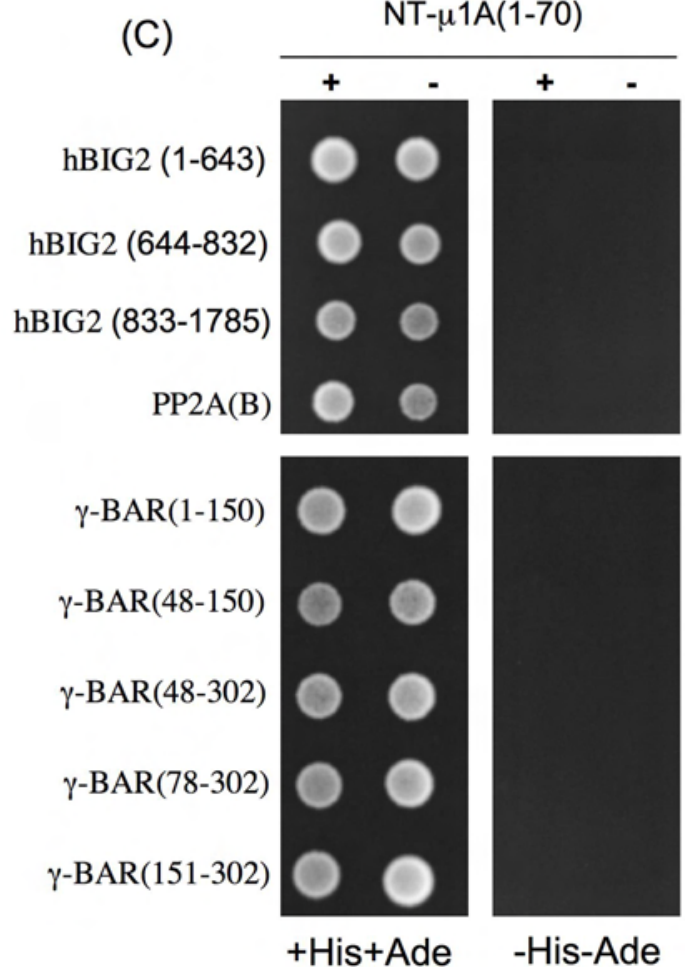

(B)

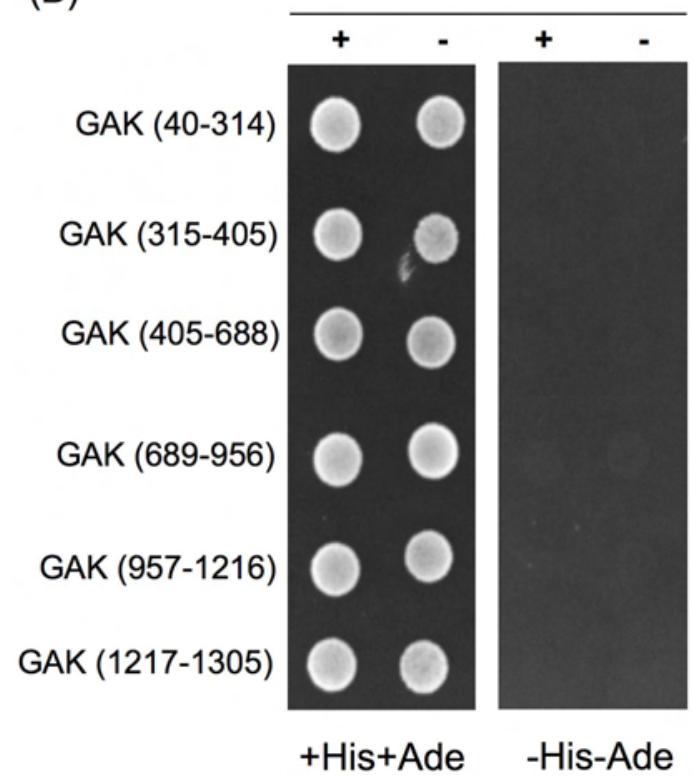

Figure 3.23: $\mathrm{Y} 2 \mathrm{H}$ analysis of $\mathrm{N}$-terminal $\mu 1 \mathrm{~A}$ with known candidate proteins. Different domains of BIG2, GAK, $\gamma$-BAR and full length PP2A-B subunit expressed from prey vector and $\mathrm{N}$-terminal seventy aminoacids of $\mu 1 \mathrm{~A}(\mathrm{NT}-\mu 1 \mathrm{~A}(1-70))$ expressed from bait vector were analyzed for interaction. Growth of co-transformants in selection medium lacking histidine and adenine (-his,-ade) indicates an interaction as depicted in (A) which served as a positive control. (B) Interaction assay with GAK domains and interaction assay with hBIG2, PP2A-B subunit and $\gamma$-BAR is shown in (C).

growth in the reporter selection plates. This indicates that the N-terminal seventy amino acids of $\mu 1 \mathrm{~A}$ do not interact with these proteins.

\subsubsection{Identification of proteins binding to the N-terminal sev- enty amino acids of $\mu 1 \mathrm{~A}$}

In addition to testing candidate proteins, we performed a library scale $\mathrm{Y} 2 \mathrm{H}$ screening using N-terminal seventy aminoacids of $\mu 1 \mathrm{~A}$ as the bait and an embryonic cDNA library prepared from 11.3 days old mouse embryo to identify new proteins involved in AP-1 regulation. A total of $\sim 10^{6}$ clones were screened and the initial 


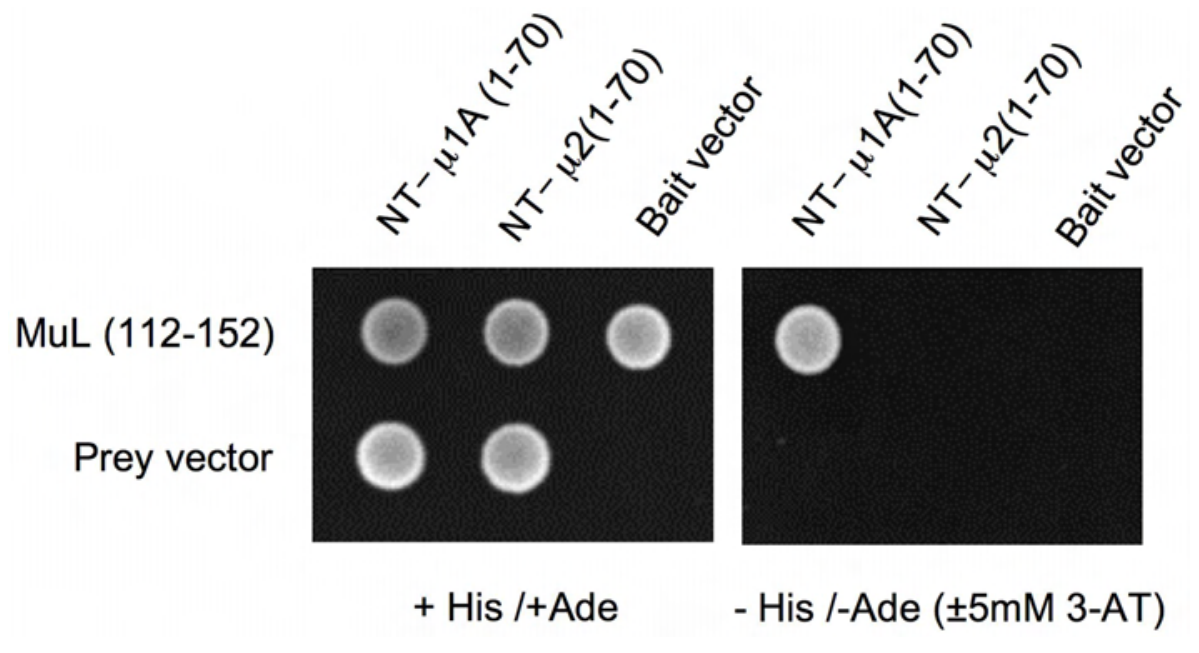

Figure 3.24: Comparison of MuL(112-195) interaction with $\mathrm{N}$-terminus $\mu 1 \mathrm{~A}$ and $\mu 2$. $\mathrm{N}$-terminal seventy amino acids of $\mu 1 \mathrm{~A}$ and $\mu 2$; NT- $\mu 1 \mathrm{~A}(1-70)$ and NT- $\mu 2(1-70)$ respectively, were used as baits to analyze their interaction with $\operatorname{MuL}(112-195)$. The growth of the cotransformants in medium lacking histidine and adenine(-His/-Ade) either in the presence or absence of $5 \mathrm{mM}$ 3-AT were monitored for 3-5 days.

screen identified 44 clones positive for all four reporter genes of the AH109 tester strain. cDNA isolated from all the putative positive clones were sequenced, which resulted in 16 unique clones. One clone containing an insert of 275 bp was identified 18 times and exhibited a strong interaction. Upon retesting these 16 clones, for confirmation of interaction by re-transformation, only the clone that exhibited strong interaction in the initial screen was truly positive. A BLAST search of this cDNA sequence against the mouse genome identified an unnamed and uncharacterized protein of 725 amino acids in length. The protein was designated MuL for $\mu$-Ligand.

To test for the specificity of the identified interaction between MuL(112-195) and N-terminal seventy amino acids of $\mu 1 \mathrm{~A}$ (NT- $\mu 1 \mathrm{~A}(1-70)$ ), we performed a Y2H interaction assay between $\mathrm{MuL}(112-195)$ and $\mathrm{N}$-terminal seventy amino acids of $\mu 2$-adaptin (NT- $\mu 2(1-70)$ ). As shown in Fig. 3.24, NT- $\mu 1 \mathrm{~A}(1-70)$ interacted with MuL(112-195), but NT- $\mu 2(1-70)$ did not. This indicates that MuL(112-195) interacts specifically with NT- $\mu 1 \mathrm{~A}(1-70)$ and the interaction is very strong as indicated by the growth of yeast in selection media supplemented with $5 \mathrm{mM} 3$ AT, an inhibitor for the expression of the product of one of the reporter genes, HIS3. Absence of growth with respective empty bait and prey vectors excluded the possibility of an auto-activation of reporter genes and thereby validating that the observed interaction is true (see Fig. 3.24).

As MuL exhibits highly specific and strong interaction with N-terminal seventy 
(A)

MuL 1

MuL(112-195)

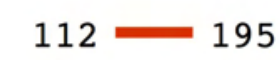

(B)

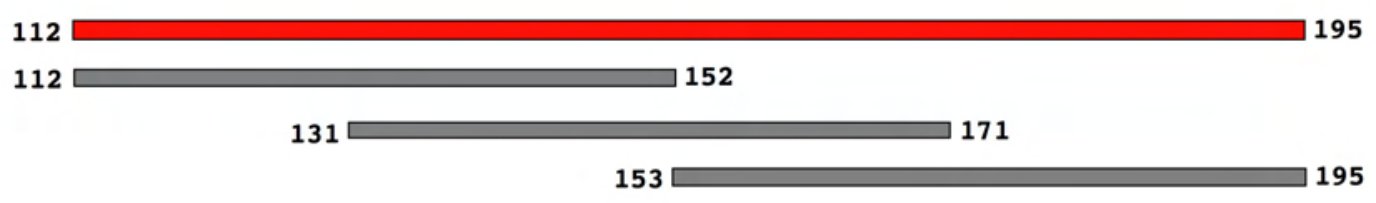

(C)

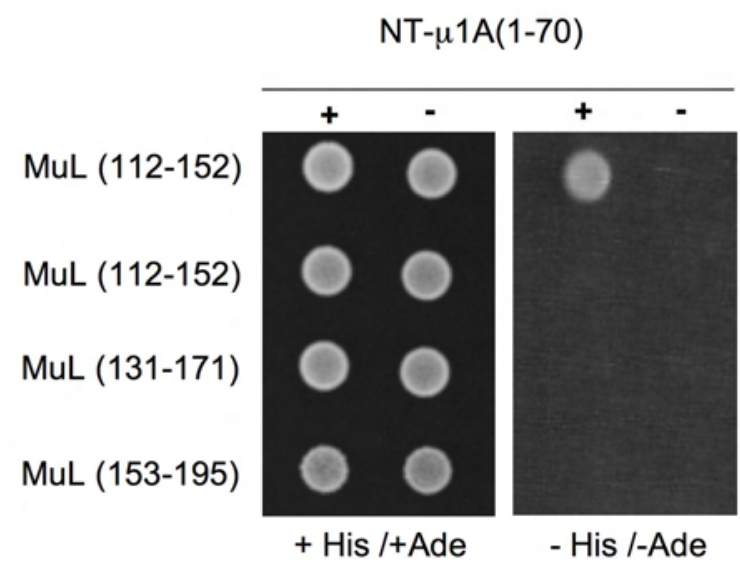

Figure 3.25: Interaction of N-terminal seventy aminoacids of $\mu 1 \mathrm{~A}$ with MuL(112-195) fragments. (A) Schematic representation of the fished $\mathrm{MuL}(112-195)$ with respect to the full length protein. (B) Schematic representation of the overlapping fragments of MuL(112195), designed to map the region of interaction with $\mathrm{N}$-terminal seventy aminoacids of $\mu 1 \mathrm{~A}$ $(\mathrm{NT}-\mu 1 \mathrm{~A}(1-70))$. (C) $\mathrm{Y} 2 \mathrm{H}$ analysis of interaction between MuL fragments and (NT- $\mu 1 \mathrm{~A}(1-70)$. The cDNAs of MuL(112-195)or its fragments with overlapping regions as shown in (B) were cloned into prey vector and analyzed for interaction with (NT- $\mu 1 \mathrm{~A}(1-70)$. The growth of the co-transformants in medium lacking histidine and adenine(-His/-Ade) were monitored for 3-5 days.

amino acids of $\mu 1 \mathrm{~A}$, we attempted to map the region that confers specificity. For this purpose, overlapping fragments of MuL(112-195) were checked for interaction with $\mathrm{N}$-terminal seventy aminoacids of $\mu 1 \mathrm{~A}$. As shown in Fig. 3.25 (C), interaction was observed only with MuL whereas none of the MuL(112-195) fragments exhibited interaction, indicating that a putative interacting motif formed by residues from all three fragments mediates the binding. 


\subsubsection{Analysis of the steady state distribution of AP-1/AP-1* in MuL-HA expressing cells}

To validate the physiological relevance of the highly specific interaction between MuL(112-195) and N-terminal seventy aminoacids of $\mu 1 \mathrm{~A}$, observed in our $\mathrm{Y} 2 \mathrm{H}$ interaction studies, we analyzed the intracellular distribution of AP- 1 and AP- $1^{*}$ in control and Chimera I (Chi-I) cells respectively, ectopically expressing MuL-HA. The complete cDNA of MuL was generated from mouse brain total RNA, by RT-PCR and was cloned into mammalian expression vector (pcDNA3) to be expressed with HA-tag at its C-terminus. The MuL-HA construct was transiently transfected in AP1 (control) and AP-1* expressing cells and analyzed by indirect immunofluorescence microscopy.

MuL-HA exhibited a cytoplasmic distribution in both control and Chi-I cells (Fig.3.26). When we analyzed for the steady-state distribution of AP-1 and AP-1* in cells that showed expression of MuL-HA, there was a remarkable reduction in the staining for $\gamma 1$-adaptin at the peri-nuclear area compared to control cells (Fig. 3.26 A, B, C, D) indicating that the recruitment of AP-1 onto membranes is disturbed in MuL-HA over-expressing cells. As expected, AP- $1^{*}$ distribution was unaltered in the presence of MuL-HA expression (Fig. 3.26 E, F, G, H). The observation that MuLHA disturbs the membrane binding of AP- 1 , but not that of AP- $1^{*}$, substantiates the specificity of interaction observed in $\mathrm{Y} 2 \mathrm{H}$ studies and also implicates that MuL plays a role in the membrane-cytoplasm recycling dynamics of AP-1. 


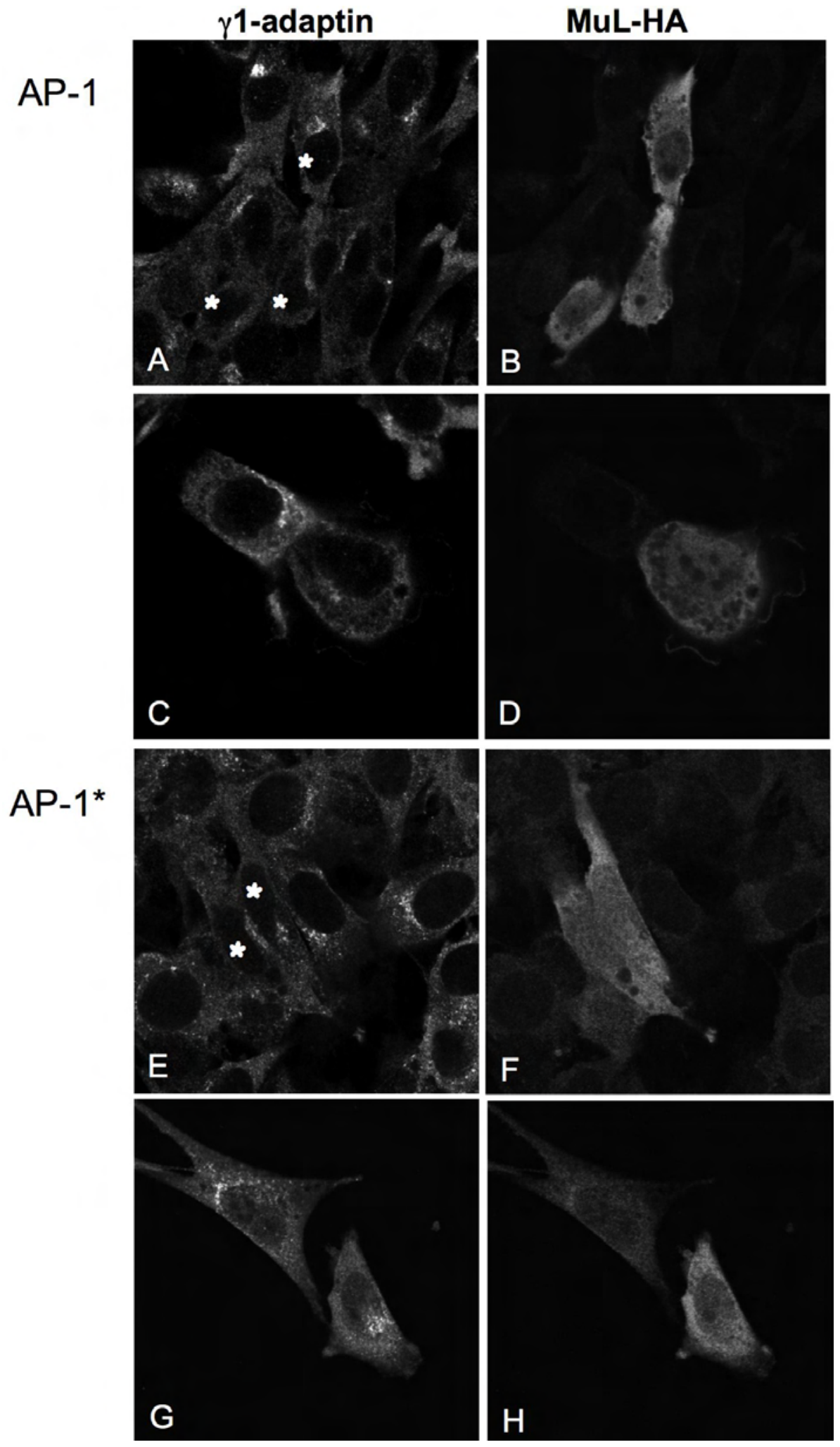

Figure 3.26: Steady-state distribution of AP-1/AP-1* in MuL-HA expressing cells. Control (AP-1) and Chimera I (AP-1*) mouse embryonic fibroblasts transiently transfected with MuLHA were grown on coverslips and fixed with 3\% PFA, 24 hours post-transfection. Permeabilized with $0.5 \%$ saponin and double immuno-stained with mouse anti- $\gamma 1$-adaptin and rat anti-HA monoclonal antibodies which were then decorated with anti-mouse IgG-Alexa 488(green) and anti-rat $\lg G$ Cy3 (red). Note the loss of $\gamma 1$-adaptin staining in MuL-HA expressing control cells indicated by astriek. 



\section{Chapter 4}

\section{Discussion}

\subsection{AP-1 in intracellular sorting of Sortilin}

At the end of the biosynthetic pathway, a subset of newly synthesized proteins are sorted to their destinations by clathrin-coated vesicles (CCVs). The first step in this pathway, is the transport of membrane-bound cargo from the TGN, the main sorting station to the sorting substation, the early endosomes. So far, AP-1 and GGAs are known to be the main players acting in this process of CCV mediated transport from the TGN to endosomes. A pioneering study with AP-1-deficient mice ( $\mu 1$ A-adaptin 'knock-out') demonstrated, that AP-1 mediated also the retrograde transport of membrane-bound receptor MPR46 (Meyer et al., 2000). The discovery of the role of monomeric receptors, the GGAs, in the anterograde transport of the MPRs, questioned the role of AP-1 in the anterograde pathway, although till now there is no evidence that GGAs are involved in the retrograde transport. Failure to identify the GGAs in CCV preparations and subsequent structural studies on GGAs, led to the proposal that the GGAs1 and 3 may act mainly in concentrating the membrane-bound cargo and sub-sequentially transfer the cargo to AP-1 in a GGA phosphorylation/de-phosphorylation cycle dependent fashion. However, GGA2 lacks such a phosphorylation cycle. The precise role of AP-1 and GGAs at the TGN remains elusive.

Recently, the GGAs were shown to be involved in the transport of a newly identified membrane-bound receptor, sortilin. Sortilin belongs to a growing family of Vps10p-domain containing, multi-ligand type I receptors involved in intracellular sorting of various ligands. It was shown that the cytoplasmic tail of sortilin contained bonafide intracellular sorting signals (Nielsen et al., 2001). The primary sequence of the cytoplasmic domain of sortilin is closely related to the cytoplasmic domain of MPR300 (cation-independent mannose 6-phosphate receptor), with a 
conserved tyrosine-based and a di-leucine based motif. The authors also reported that the sortilin cytoplasmic domain interacted with GGA2, a member of the GGA family of monomeric adaptors that are involved in the transport of cargo from the TGN to the endosome, concluding that the transport of sortilin from the TGN to endosomes is mediated by GGA2 (Nielsen et al., 2001). But surprisingly, so far, all of the reported studies on sortilin transport, did not address the role of AP- 1 in the transport of sortilin.

In the present study, we examined the role of AP-1 in the intracellular sorting of sortilin. Previous studies from our group, based on AP-1-deficient mice ( $\mu 1 \mathrm{~A}$ adaptin 'knock-out') has demonstrated the involvement of AP-1 in the transport of MPR300. Since sortilin exhibits similar sorting characteristics as of MPR300, we were interested to know the fate of sortilin in AP-1-deficient cells.

The mannose 6-phosphate receptors MPR46 and MPR300 are sorted into clathrin-coated vesicles by AP-1. Deficiency of AP-1 results in a redistribution of the receptors from the TGN, their main localization at steady-state, to earlyendosomal compartments as observed in $\mu 1 \mathrm{~A}$-/- fibroblasts (Meyer et al., 2000). We analyzed the intracellular distribution of sortilin in AP-1-deficient MEFs by indirectimmunofluorescence microscopy (Fig. 3.1). As previously shown (Meyer et al., 2000), in $\mu 1 \mathrm{~A}$-/- cells, $\gamma 1$-adaptin staining is lost or very weak at the TGN with more peripheral distribution of MPR300 compared to control cells, where both show overlapping staining at the TGN. Sortilin was shown to be predominantly localized at the TGN and vesicles with an extensive co-localization with MPR300 (Petersen et al., 2001). In agreement with this, the distribution of sortilin and MPR300 (Fig. $3.1 \mathrm{G}$ and $\mathrm{H}$ respectively) in control cells was mainly peri-nuclear and overlapped extensively (Fig. $3.1 \mathrm{I}$ ). But in $\mu 1 \mathrm{~A}$-/- cells, there was a remarkable distribution of sortilin mainly to peripheral structures (Fig. $3.1 \mathrm{~J}$ ) with dispersed staining, overlapping extensively with re-distributed MPR300 (Fig. 3.1 L). Since MPR300 is known to re-distribute to early endosomal structures in $\mu 1 \mathrm{~A} \mathrm{-/-} \mathrm{cells}$ (Meyer et al., 2000), this co-localization implies that sortilin is also re-distributed to early-endosomes in a similar way as MPR300. Sortilin antibody endocytosis experiments demonstrated that sortilin is mis-localized to endosomes as it has been shown for both manose 6-phosphate receptors.

Although sortilin redistributed to MPR300 positive endosomes in $\mu 1 \mathrm{~A}$-/- cells, its staining appeared even more widely dispersed. To ascertain whether sortilin is mis-sorted to late endosomes or lysosomes, we performed co-staining with LAMP1, a marker for late endosomes and lysosomes. As shown in Fig.3.2 (D,E and F), sortilin is found in dispersed LAMP-1 negative structures, implying that sortilin is not mis-sorted to lysosomes. AP-3 is another member of the heterotetrameric adaptor 
complexes, which has been shown to localize at the TGN and endosomes, but with a predominant localization at the early endosomes (Reusch et al., 2002). To validate whether AP-3 is involved in the sorting events of sortilin, we analyzed the steadystate distribution of sortilin in AP3-/- cells, which are deficient for $\delta$-3 adaptin. Sortilin stained predominantly in the peri-nuclear area as observed in control cells (Fig. 3.2 A) implying that its steady-state distribution is unaltered in the absence of AP-3.

\section{Sortilin - AP-1 interactions}

The observed difference in the steady-state localization of sortilin in $\mu 1 \mathrm{~A}-/-$ cells prompted us to check, whether the sortilin tail could mediate direct interaction with $\mu 1 \mathrm{~A}$. The sortilin CD contains two sorting motifs: a tyrosine-based motif and an acidic di-leucine motif (Fig. 3.3) and both are able to mediate binding to AP-1 and AP-2 complexes as shown for MPR300 and many other proteins. We used the established $\mathrm{Y} 2 \mathrm{H}$ method to check for possible interaction, as this system has proven to be successful in identifying even weak interactions. Two different constructs of sortilin cytoplasmic domain(CD) were used in this analysis; the full length CD (sortilin CD) and a truncated form lacking the tyrosine-based motif, but containing the di-leucine motif (sortilin $C D(\Delta 1-30)$ ). These two different sortilin CDs were checked for interaction with the C-terminal domain (150-450 aa) of $\mu 1 \mathrm{~A}$ ( $\mu 1 \mathrm{~A} \mathrm{CT}$ ) that contains the $\mathrm{Yxx} \phi$ sorting motif binding site.

Our data indicate that sortilin CD interacts directly with $\mu 1 \mathrm{~A} \mathrm{CT}$ albeit weakly. This interaction is most likely mediated by the tyrosine-based motif, as the sortilin CD lacking this motif failed to show even weak interaction (Fig. 3.4). MPR46 CD exhibited auto-activation and so its interaction could not be validated. However, MPR300 CD showed a strong interaction with $\mu 1 \mathrm{~A} \mathrm{CT}$. This apparent difference in the affinities could be explained in two ways. First, it has been shown that the MPR300 cytoplasmic tail contains four binding sites for AP-1 (Ghosh and Kornfeld, 2004). Using GST pull-down assay, the authors identified four binding motifs: a tyrosine -based motif (26YSKV29), an internal di-leucine based motif (39ETEWLM44), and two casein kinase 2 sites (84DSEDE88 and 154DDSDED159); YSKV motif mediated the strongest interaction with AP-1 and the two CK2 motifs bound AP-1 only when they were phosphorylated. Also, the authors had shown that the COOH-terminal di-leucine motifs were not required for interaction with AP1. However other studies using surface plasmon resonance to test for AP-binding indicate that AP-1 as well as AP-2 are able to bind to the C-terminal di-leucine motif. Therefore, one can speculate that binding to more than one site, to both tyrosine- 
based and internal di-leucine based motif of MPR300 cytoplasmic domain, leads to the increased affinity in our yeast two-hybrid assay.

Secondly, the nature of the tyrosine based ( $\mathrm{Yxx} \phi$; Y-tyrosine, $\mathrm{x}$-any aminoacid, $\phi$-residues with bulky side chain) sorting signal may be responsible for different affinities. There is substantial evidence that $\operatorname{Yxx} \phi$ motif is the minimal requirement to confine recognition by the $\mu$-adaptins. However, the $\mathrm{X}$ residues and other residues flanking the motif also contribute to the strength of interaction and fine tuning of the specificity of the signals (Bonifacino and Traub, 2003). The Y residue is essential for function and in most cases cannot even be substituted by other aromatic amino acid residues, suggesting that the phenolic hydroxyl group of the tyrosine is a critical recognition element. The $\phi$ position can accommodate several residues with bulky hydrophobic side chains, although the exact identity of this residue can specify the properties of the signal. The $\mathrm{X}$ residues are highly variable, but tend to be hydrophilic. Such preferences are not clearly known for recognition by $\mu 1 \mathrm{~A}$, but at least for $\mu 2$ it has been shown that mutations of the X-residues tend to decrease the strength of interaction. In the case of MPR300 and sortilin with YSKV and YSVL motifs respectively, the only obvious difference is the presence of valine(V) at $\mathrm{Y}+2$ position in sortilin, a small residue with less hydrophilicity compared to lysine(K) in MPR300. Introduction of mutations to mimic either of the residues could provide an answer for this difference in affinities. In addition, the influence of flanking residues needs to be tested.

As a next step, we performed a GSTpull-down assay using GST-sortilin CD fusion proteins and cytosol preparations from control and $\mu 1 \mathrm{~A}-/$ - cells. GST-Sortilin CD efficiently pulled-down AP-1 from the control(ct) cytosol compared to GST-Sortilin $\mathrm{CD}(\Delta 1-30)$ that lacks the tyrosine-based motif (Fig. 3.6). This confirms that the tyrosine-based motif is sufficient for interaction between sortilin CD and $\mu 1 \mathrm{~A}$, as observed by $\mathrm{Y} 2 \mathrm{H}$ interaction study. Yet a weak signal for $\gamma 1$ was observed in the pull-down fraction of GST-Sortilin $\operatorname{CD}(\Delta 1-30)$, which means that the interaction is not completely lost.

Surprisingly, similar results were observed, when cytosol from $\mu 1 \mathrm{~A}$-/- cells was used for pull-down assays (Fig. 3.6). Apparently GST-Sortilin CD was able to pulldown $\gamma 1$-adaptin, indicating that sortilin could bind to $\gamma 1$, or most likely to a $\gamma 1$ $\sigma 1$ hemi-complex, independent of $\mu 1 \mathrm{~A}$. It has been shown that a trimeric complex consisting of a $\gamma 1-\sigma 1$ hemi-complex and $\beta 1$ adaptin still exists in $\mu 1 \mathrm{~A}-/$ - fibroblasts (Meyer et al., 2000). However, this trimeric complex is non-functional in protein sorting because it does not bind to membranes in vivo, and in vitro. So, it is possible that sortilin CD interacts with the cytosolic $\gamma 1-\sigma 1-\beta 1$ complex present in $\mu 1 \mathrm{~A}-/-$ cells. A recent study has shown that $\gamma 1-\sigma 1 \mathrm{~A}$ hemi-complex interacts with acidic 
di-leucine motif present in the cytosolic tails of HIV-Nef and LIMP-II (Janvier et al., 2003). Therefore, one can expect that the interaction between sortilin and the $\gamma 1$ $\sigma 1$ hemi-complex could be mediated by the acidic di-leucine motif present in sortilin $\mathrm{CD}$, although only a weak signal for $\gamma 1$ was observed in the pull-down fraction of GST-Sortilin $C D(\Delta 1-30)$. One possible explanation to this discrepancy could be that the acidic di-leucine motif in GST- Sortilin $\operatorname{CD}(\Delta 1-30)$ may not be completely accessible for interaction due to steric hinderance or due to the requirement of a post-translational modification. In addition, that in $\mu 1 \mathrm{~A}-/-$ cells the remaining adaptins are not present in a 1:1:1 ratio. While $\gamma 1$ is slightly reduced to $70 \%$ of wt levels, $\sigma 1$ is reduced to $30 \%$. Thus, reduced complex stability could contribute to the low pull-down efficiency.

We tested for interaction between sortilin and the hemi-complexes containing $\gamma 1$ and various isoforms of $\sigma 1$ including different splice variants for the $\sigma 1 \mathrm{~B}$ gene, identified in a previous study of our lab (Riel, 2004). The splice variants exhibited tissue specific expression pattern. $\sigma 1 \mathrm{~B} 2$ and $\sigma 1 \mathrm{~B} 3$ are expressed mainly in the brain and skeletal muscle respectively. Both sortilin CD and sortilin $C D(\Delta 1-30)$ interacted with the $\gamma 1-\sigma 1$ hemi-complex (Fig. 3.7 A). Surprisingly, this interaction was restricted only to the hemi-complex containing the $\sigma 1 \mathrm{~B}$ isoform and its splice variants $\sigma 1 \mathrm{~B} 2$ and $\sigma 1 \mathrm{~B} 3$. But, $\sigma 1 \mathrm{~A}$ and $\sigma 1 \mathrm{C}$ containing hemi-complexes showed extremely weak or no interaction. It has to be noted that in the study of Janvier et al.,2003, the acidic di-leucine motif of the type D/EXXXL(L/I) present in the cytosolic tails of HIV-Nef and LIMP-II interacted with $\gamma 1-\sigma 1 \mathrm{~A}$ hemi-complex, but interaction with hemi-complexes containing either $\sigma 1 \mathrm{~B}$ or $\sigma 1 \mathrm{C}$ were not tested. This high affinity di-leucine based motif did not show any $\sigma 1$ - specificity, indicating low affinity di-leucine based signals are required to allow specific binding to a specific AP-1 complex in vivo.

Further, it was interesting to note different affinities of sortilin to the $\sigma 1 \mathrm{~B}$ splice variants. The interaction between $\sigma 1 \mathrm{~B} 2$ containing hemi-complex and sortilin $\mathrm{CD}(\Delta 1-30)$ was stronger than that of $\sigma 1 \mathrm{~B} 1$ containing hemi-complex, as evident from the difference in growth rate of the yeast clones (Fig. 3.7 A). Of note, this specificity correlates with the tissue expression pattern of sortilin and of $\sigma 1 \mathrm{~B}$ splice variants. Sortilin was shown to be mainly expressed in brain, skeletal muscle and adipocytes. In a seperate experiment, we checked for interaction between sortilin and $\gamma 1$ or $\sigma 1$ alone, but no interaction was observed. The interaction observed only with the hemi-complex implies that both the subunits form the binding site for interaction with sortilin.

After observing an interaction between sortilin and the $\gamma 1-\sigma 1 \mathrm{~B}$ hemi-complex by $\mathrm{Y} 3 \mathrm{H}$ assay, we performed GST pulldown assays to see whether we can observe 
the interaction in vitro in cells that are deficient for $\sigma 1 \mathrm{~B}$ adaptin. The $\sigma 1 \mathrm{~B}$ deficient mouse embryonic fibroblasts were obtained from a $\sigma 1 \mathrm{~B}$ 'knock-out' mouse generated in our lab (Riel, 2004). MEFs normally express $\sigma 1 \mathrm{~A}$, -B and -C. GSTSortilin CD was able to pull-down AP-1 from the $\sigma 1 \mathrm{~B}-/$ - cytosol, but no interaction with either GST-Sortilin $\mathrm{CD}(\Delta 1-30)$ or GST alone (Fig. 3.8), was detected. This was not surprising, because sortilin $\mathrm{CD}$ is able to bind to $\mu 1 \mathrm{~A} \mathrm{CT}$ through the tyrosine-based motif (Fig. 3.4). Thus this motif appears to be responsible and thus sufficient for the pull-down of AP-1 by sortilin CD. In all pull-down experiments, the contribution of the di-leucine motif is barely detectable, indicating a low-affinity interaction. This observation substantiates the idea that sortilin CD interacts with AP-1 via both the tyrosine based and acidic di-leucine motifs.

The observed interaction between the sortilin di-leucine based motif and $\gamma 1-\sigma 1 \mathrm{~B}$ hemi-complex prompted us to check for the steady-state distribution of sortilin in $\sigma 1 \mathrm{~B}-/$ - fibroblasts by indirect immunofluorescence microscopy. We were unable to see any change in the steady-state distribution of sortilin in $\sigma 1 \mathrm{~B}-/-$ fibroblasts. The staining of sortilin was unaltered with predominant co-localization with MPR300 at the peri-nuclear region. This indicates that binding to $\mu 1 \mathrm{~A}$-adaptin is sufficient to mediate TGN - endosome sorting in the mouse embryonic-fibroblasts. However traffic on additional routes could still be mediated by $\sigma 1 \mathrm{~B}$ in the respective tissues. Examination of the steady-state distribution of sorting-motif mutants of sortilin further substantiates our finding that AP- 1 is involved in the transport events of sortilin between the endosomes and the TGN. We followed steady-state distribution of IL2R-sortilin CD mutant chimeras in the control and $\sigma 1 \mathrm{~B}-/$ - fibroblasts (Fig. 3.10 and Fig. 3.11). The IL2R-sortilin CD-Y14A mutant and also the -Y14A/L17A mutant are mis-sorted to peripheral structures, indicating that the tyrosine-residue is the most important for $\mu 1 \mathrm{~A}$ mediated transport, and that this binding mediates endosomes to TGN transport. IL2R-sortilin CD- $\Delta$ LL exhibited an absolute localization at the peri-nuclear compartment with little or no distribution in vesicular structures in contrast to sortilin-wt in control cells. This could indicate an impaired exit of IL2R-Sortilin CD- $\Delta \mathrm{LL}$ receptor at the TGN, implicating the significance of dileucine in transport of the receptor from the TGN. However, the mutant sortilin CD-Y14A/L17A $+\Delta$ LL exhibited a mixed pattern with prominent localization at the plasma membrane and also with considerable peri-nuclear and vesicular staining. This indicates that the $\Delta \mathrm{LL}$ mutant is able to leave the TGN, presumably by constitutive exocytosis. Both motifs however appear to be required for endocytosis, mediated by the homologous AP-2. All other sortilin mutants: sortilin CD-S47D, -S47A, -G42A/Y43A, -D45A/D46A and -D48A/E49A/D50A showed a predominant localization at the peri-nuclear compartment, a pattern as exhibited by sortilin-wt 
in control cells, indicating that they are dispensible for the intracellular sorting of sortilin.

\subsubsection{Perspective}

Our finding that the di-leucine based motif interacts specifically with $\gamma 1-\sigma 1 \mathrm{~B}$ hemicomplex leads to an intriguing possibility of the existence of a sub-population of AP-1, containing variants of $\sigma 1 \mathrm{~B}$-adaptins that mediate the sorting events involving sortilin, in specialized tissues. The similarity in the expression pattern of $\sigma 1 \mathrm{~B}$ and its splice variants and of sortilin substantiates this idea. In a recent study, it was shown that sortilin is essential for the biogenesis of Glut4 storage vesicles in 3T3L1adipocytes (Shi and Kandror, 2005). In the absence of sortilin, it has been shown that the Glut4 transporter is rapidly degraded. Moreover, it has been shown that AP1 is associated with Glut4 vesicles (Gillingham et al., 1999). However, AP-3 was also found to be associated to these vesicles. In addition, it was proposed that sortilin sorting by GGA2 mediates Glut4 sorting via sortilin-Glut4 dimerization. The data presented in this thesis, however, indicate that AP- $1 / \sigma 1 \mathrm{~B}$ complexes might play a role in sortilin sorting and thus Glut4 vesicle biogenesis. Currently experiments are performed to test this hypothesis using tissues cultured from the $\sigma 1 \mathrm{~B}$ 'knock-out' mouse. 


\subsection{A role for the $\mathrm{N}$-terminal domain of $\mu 1 \mathrm{~A}$ in regu- lation of AP-1 function}

AP-1, a heterotetrameric clathrin adaptor complex, is mainly involved in the clathrin coated vesicle mediated transport of membrane receptors and their cargo between the TGN and the endosomes. In this study, we performed a comprehensive analysis of the $\mathrm{N}$-terminal domain of $\mu 1 \mathrm{~A}$ and present data demonstrating that its $\mathrm{N}$-terminal seventy amino acids play a role in the regulation of the sorting function of AP-1 complex.

\subsubsection{Influence of the $\mathrm{N}$-terminal domain of $\mu 1 \mathrm{~A}$ on AP-1 medi- ated protein sorting}

Studies on $\mu 2 / \mu 1$-chimeric AP- 1 complex (AP- $1^{*}$ ) provided the first evidence for a role of the $\mathrm{N}$-terminal domain of $\mu 1 \mathrm{~A}$-adaptin in protein sorting (Medigeshi Ramarao, 2003), Guruprasad Medegeshi and Schu.P, unpublished). The $\mu 2^{1-70} / \mu 1^{71-423}$ chimera (Chimera I, Fig.3.15) was incorporated into an AP- $1^{*}$ complex, which localized with clathrin and with the mannose-6-phosphate receptor at the perinuclear TGN. However AP-1* was able to restore mannose-6-phosphate receptor mediated cathepsin D sorting only partially. Also, redistribution of AP-1* into the cytoplasm induced by Brefeldin A was slower than that of AP-1. Cryo-electron microscopic analysis of AP-1* expressing cells, in the present study, displayed enlarged early endosomal organelles as observed in AP-1-deficient ( $\mu 1 \mathrm{~A}-/-)$ cells indicating an imbalance in membrane trafficking (Fig.3.17 B and C).

In the $\mu 1 \mathrm{~A}$ 'knock-out' cells, peri-nuclear TGN staining of $\gamma 1$-adaptin is lost and also clathrin staining is severely reduced. The mannose-6-phosphate receptors MPR46 and MPR300, which mediate sorting of lysosomal enzymes between the TGN and endosomes are redistributed from their preferential peri-nuclear TGN localization to early endosomes. This is due to a block in retrograde endosome to TGN transport (Meyer et al., 2000, 2001). Although, AP-1* was able to restore the predominant TGN localization of mannose-6-phosphate receptors, TGN to endosome sorting of MPRs is impaired as indicated by the secretion of cathepsin D. That AP-1* cells still display enlarged endosomes also demonstrates impaired endosomal trafficking. These data demonstrate that the $\mathrm{N}$-terminal seventy amino acids of $\mu 1 \mathrm{~A}$ adaptin are important for AP-1 mediated sorting of membrane receptors and their cargo. 


\subsubsection{Role in AP-1 membrane binding dynamics}

Having provided evidence that the N-terminal seventy amino acids of $\mu 1 \mathrm{~A}$-adaptin could be involved in the regulation of AP-1 mediated vesicle transport, we studied the membrane recycling kinetics of AP- $1^{*}$. It has been demonstrated that clathrincoated pits are dynamic structures wherein the membrane bound clathrin and adaptors, dynamically exchange with their free counterparts in the cytoplasmic pool, also independent of eachother and that the adaptors exchange independent of vesicle budding. This has been demonstrated for AP-1 and AP-2 (Wu et al., 2001, 2003). By FRAP analysis, we studied the nature of AP-1 and AP-1* cytoplasm membrane recycling kinetics at the TGN, to gain insight into their varied response to Brefeldin A treatment. Our data show that the rate of exchange of AP-1* between the membrane bound fraction and the cytoplasmic pool is reduced. Although the membrane bound AP-1 and AP- $1^{*}$ were able to exchange with the cytoplasmic pool of adaptors as evidenced by the complete recovery of fluorescence at the photobleached area (see Fig. 3.18), AP- $1^{*}$ exhibited slow recycling kinetics, with almost a 50\% reduction in its recycling rate compared to AP-1(Fig.3.19 A). When vesicle budding was blocked by treatment with hypertonic sucrose, AP-1* exchanged slowly with the cytoplasmic pool (Fig. 3.19 B) as did AP-1, but the difference in recovery remained at $50 \%$. However the total recovery of AP- $1^{*}$ fluorescence was reduced to about $\sim 60 \%$, whereas recovery of AP-1 was $\sim 90 \%$ also under these conditions. These data demonstrate that in the absence of vesicle budding process, a larger fraction of AP- $1^{*}$ binds to the membrane more firmly than AP-1, presumably at non-productive sites leading to reduced recycling.

The membrane recruitment of AP-1 is regulated by cycles of phosphorylation and de-phosphorylation of the $\beta 1$-and $\mu 1$-adaptins (Ghosh and Kornfeld, 2003a). It was shown that the $\beta 1$-adaptin is phosphorylated in the cytoplasm, but will be de-phosphorylated, when AP-1 is bound to the membrane, whereas $\mu 1 \mathrm{~A}$ is in the de-phosphorylated state in the cytoplasm, but phosphorylated in the membrane bound state. A model has been developed based on two observations. Firstly, upon $\mu$-phosphorylation, AP-cargo binding is enhanced and secondly, in the AP-complex, the cargo-sorting motif binding site of $\mu$-adaptin is covered by $\beta$-adaptin. Therefore, it was proposed that the phosphorylation of $\mu$ induces high affinity binding to the cargo sorting motif, by facilitating or stabilizing a conformational change in $\mu$, that exposes its sorting motif binding site. This model applies to both AP-2 and AP-1. One can expect that, an impairment in this phosphorylation/de-phosphorylation cycle could lead to altered membrane binding properties of AP-1. Since our FRAP data revealed an impairment in the membrane-cytoplasm recycling of AP- $1^{*}$, we 
were interested to look for the phosphorylation state of AP-1*.

In the phosphorylation-induced open conformation, $\mu 1 \mathrm{~A}$ is accessible for trypsin digestion in vitro. Therefore susceptibility to trypsin digestion is an indirect measure of the phosphorylation state of $\mu 1 \mathrm{~A}$ and we took advantage of this property to compare the phosphorylation state of AP-1* and AP-1. $\mu 1 \mathrm{~A}$ of membrane-bound AP-1 exhibited a dramatic dose-dependent tryptic degradation, but Chimera I of membrane-bound AP-1* was less sensitive and indicated that the unphosphroylated membrane-bound fraction of AP- $1^{*}$ is larger than that of AP-1 (Fig. 3.21). Two possibilities can be derived for the state of under-phosphorylation; either due to increased de-phosphorylation or reduced phosphorylation combined with increased phosphorylation-independent membrane/cargo binding. Of note, it has been shown that the phosphorylation event of $\mu 1 \mathrm{~A}$ is associated with clathrin coated vesicle formation and budding and its de-phosphorylation with release from membrane (Ghosh and Kornfeld, 2003a). The under-phosphorylation state, taken together with our observation from FRAP analysis, makes it likely that there is an increased phosphorylation-independent membrane binding of AP- $1^{*}$ at non-productive sites. As a consequence of this binding to non-productive sites, the fraction of the cytoplasmic pool available for productive transport vesicle formation, becomes smaller and thus limiting for transport vesicle formation.

\subsubsection{A novel protein as a potential regulator of AP-1 membrane binding}

Little is known about the mechanisms that regulate the recruitment of AP-1 onto membranes and the proteins involved in its regulation. The N-terminal seventy amino acids of $\mu 1 \mathrm{~A}$-adaptin can mediate its regulatory role by either directly recruiting the regulatory proteins or by stabilizing already recruited proteins. Given the fact that the $\mathrm{N}$-terminal seventy amino acids of $\mu 1 \mathrm{~A}$-adaptin are accessible from the cytoplasmic face of the heterotetrameric adaptor complex (Fig. 3.20), it is possible that cytoplasmic accessory and regulatory proteins involved in AP1 mediated protein transport can bind to the $\mu 1 \mathrm{~A}$ N-terminus and regulate AP-1 membrane binding.

The proteins that are shown to be involved in the membrane recruitment of AP-1, obviously define the potential candidates for interaction. Membrane bound ARF- 1 recruits AP- 1 onto membranes at the site of vesicle formation at the TGN and the events leading to receptor sorting and transport vesicle formation. BIG2 was identified as a guanine-nucleotide exchange factor (GEF) for ARF-1 operating at the TGN, which when overexpressed, blocked the Brefeldin A-induced redistribution of 
AP-1 from membranes, indicating that it is implicated in AP-1 membrane binding (Shinotsuka et al., 2002). Since we know from our Brefeldin A treatment studies that AP-1* was still found bound to the membranes, BIG2 could be an ideal candidate to test for interaction. But our yeast two-hybrid studies indicate that none of the domains tested interacts with the N-terminal seventy aminoacids of $\mu 1 \mathrm{~A}$. Similarly, the other potential candidates tested, cyclin $\mathrm{G}$ associated kinase (GAK)/ auxilin 2, the B-subunit of Protein phosphatase 2A (PP2A) and $\gamma$-BAR ( $\gamma 1$ adaptin Brefeldin A Resistance), did not show an interaction (see Fig. 3.23). This indicates that a mechanism that does not involve these proteins might operate in the N-terminal $\mu 1 \mathrm{~A}$ mediated regulation of AP-1 membrane binding.

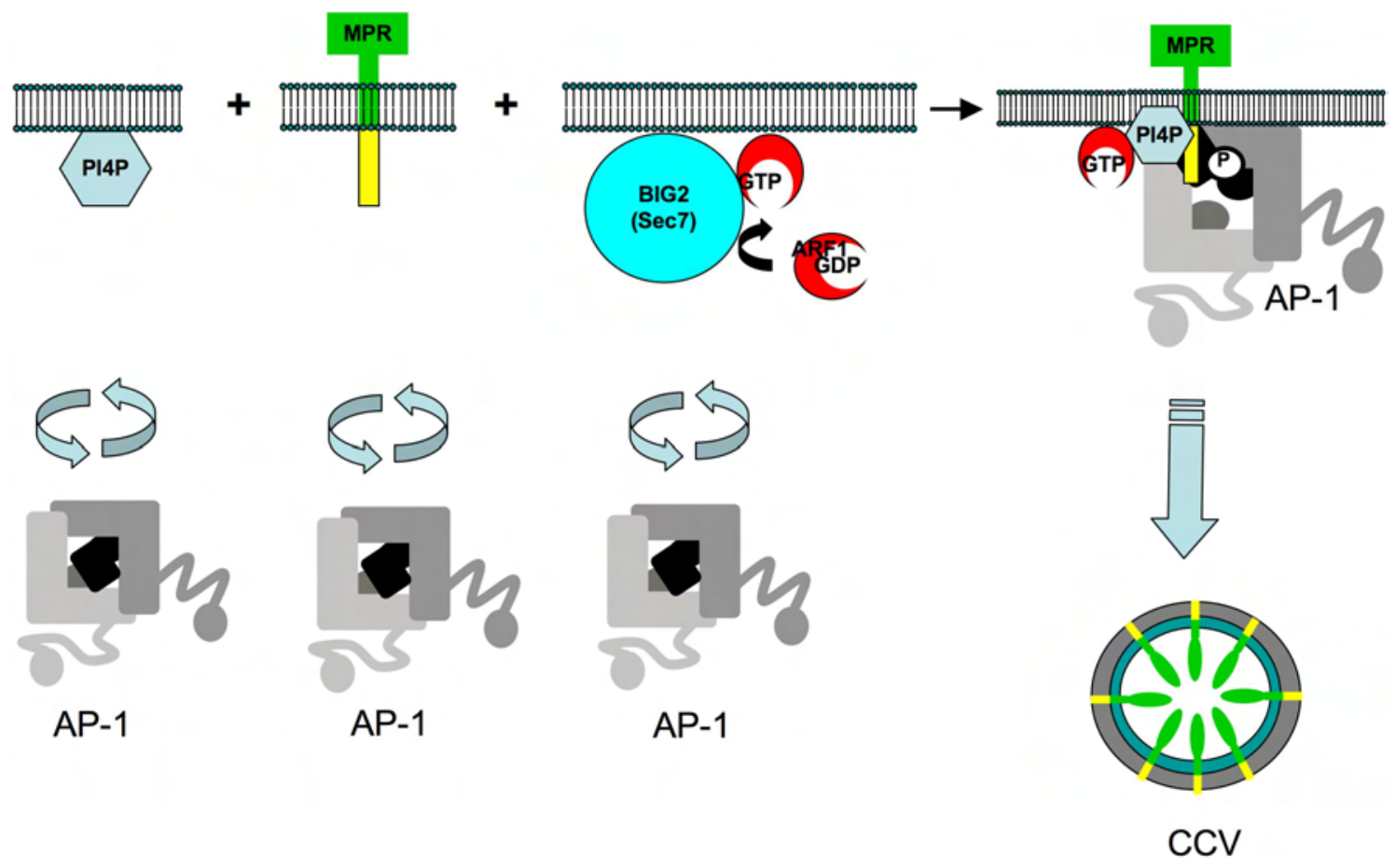

Figure 4.1: A model depicting the possible role of MuL in regulation of AP-1 membrane binding.

To identify new proteins that could potentially bind, we performed a $\mathrm{Y} 2 \mathrm{H}$ library screening using N-terminal seventy aminoacids of $\mu 1 \mathrm{~A}$ as the bait and a mouse embryonic cDNA library. We identified a fragment of an unknown and an uncharacterized protein that showed a strong interaction. This interaction was also highly specific, because it did not interact with the corresponding domain of $\mu 2$ (Fig. 3.24). When we analyzed for the steady-state distribution of AP- 1 and AP- ${ }^{*}$ in cells that showed transient expression of MuL-HA, there was a remarkable reduction in the staining for $\gamma 1$-adaptin at the peri-nuclear area compared to control cells, indicating that the recruitment of AP-1 onto membranes is disturbed in MuL-HA 
over-expressing cells. As expected, AP-1* distribution was unaltered in the presence of MuL-HA expression. The observation that MuL-HA disturbs the membrane binding of AP-1, but not that of AP-1*, substantiates the specificity of interaction observed in $\mathrm{Y} 2 \mathrm{H}$ studies and also implicates that MuL plays a role in the membranecytoplasm recycling dynamics of AP-1.

So, how could MuL mediate its regulatory role? Based on our results, we propose a model by which MuL executes its role in AP-1 membrane binding (Fig.4.1). AP-1 high affinity membrane binding requires cargo, activated ARF-1 and PI4P and all the three factors are required for vesicle formation. However, AP-1 binding by just one of these factors is sufficient to permit AP-1 membrane recruitment in vitro. MuLoverexpression phenotype indicates that AP-1 membrane binding mediated by just one of the factors also occurs in vivo, reducing the cytoplasmic, mobile AP-1 pool available for binding at sites of vesicle formation and that MuL action ensures rapid release of AP-1 from membrane sites where vesicle budding cannot take place. MuL might perform this function alone or it could act as an adaptor protein. 


\section{Summary}

The data presented in this thesis project provide the first data for the function of $\sigma 1$ adaptin isoforms and thus a function of tissue specifically expressed AP- 1 complexes. Most tissues express two of the three $\sigma 1 \mathrm{~A}, \sigma 1 \mathrm{~B}$ and $\sigma 1 \mathrm{C}$ adaptins and in addition, in some tissues, the $\sigma 1 \mathrm{~B}$-adaptin is present in isoforms generated by alternative RNA splicing. It is shown that the two previously characterized sorting motifs in the sortilin receptor are recognized by two different adaptins; the tyrosine-based motif is recognized by the ubiquitously expressed $\mu 1 \mathrm{~A}$-adaptin, whereas the dileucine based motif is specifically recognized by a $\gamma 1-\sigma 1 \mathrm{~B}$ hemi-complex. Sortilin is a recently identified multi-ligand receptor belonging to the Vps10p-domain receptor family, involved in the sorting of various unrelated ligands to the lysosomes. Similar to MPRs, it cycles between the TGN and the early and late endosomes mediated by clathrin-coated vesicles and its steady-state localization is confined to the TGN. The monomeric adaptor GGAs were shown to be responsible for mediating the TGN to endosome transport of sortilin by binding the di-leucine based sorting motif. Our study demonstrates that, AP-1 is also necessary for intracellular sorting of sortilin. The steady-state localization of sortilin in AP-1-deficient ( $\mu 1 \mathrm{~A}-/-)$ cells, is altered with a redistribution to early-endosomes in a similar way as MPRs. This indicates that AP-1 is required for the retrograde transport of sortilin from the early-endosomes back to the TGN. Although the steady-state distribution of sortilin, was unaltered in $\sigma 1 \mathrm{~B}$-deficient ( $\sigma 1 \mathrm{~B}$ 'knock-out') fibroblasts, the significance of this interaction should be studied further in other tissues, owing to the striking similarity in the tissue-specific expression pattern of sortilin and $\sigma 1 \mathrm{~B}$ splice variants, $\sigma 1 \mathrm{~B} 2$ and $\sigma 1 \mathrm{~B} 3$. These include muscle, brain and adipose tissues. Since a $\sigma 1 \mathrm{~B}$ 'knock-out' mouse had been generated in our group, which is viable, tissue specific functions can now be analyzed in the molecular level.

In a second project, the regulation of AP-1 complex membrane-recycling was studied. The AP- $1^{*}$ complex containing the $\mu 2^{1-70} / \mu 1^{71-423}$ chimera was able to bind membrane, but was not able to fully restore the phenotype of $\mu 1 \mathrm{~A}$-deficiency. AP-1* expressing cells displayed enlarged endosomes as seen in AP-1-deficient ( $\mu 1 \mathrm{~A}-/-)$ cells, which demonstrates an imbalance in membrane trafficking and thereby, the 
significance of $\mu 1 \mathrm{~A}^{1-70}$ in maintaining the fidelity of AP-1 function. AP- $1^{*}$ exhibited slow cytoplasm-membrane recycling kinetics compared to AP-1. Moreover, the data indicate that AP- ${ }^{*}$ binds to the membrane more firmly than AP- 1 and at non-productive sites. $\mu 1 \mathrm{~A}$ of membrane-bound AP-1 exhibited a dramatic dosedependent tryptic degradation, a consequence of phosphorylation-induced change in conformation and accessibility to trypsin digestion. But $\mu 2^{1-70} / \mu 1^{71-423}$ chimera of membrane-bound AP- $1^{*}$ was less sensitive, indicating that the unphosphroylated membrane-bound fraction of AP- $1^{*}$ is larger than that of AP-1. As a consequence of this binding to non-productive sites, the fraction of the cytoplasmic pool available for productive transport vesicle formation, becomes smaller and thus limiting for transport vesicle formation. Since, the N-terminal seventy amino acids of $\mu 1 \mathrm{~A}$ adaptin are accessible from the cytoplasmic face of the heterotetrameric adaptor complex, we speculated that it can execute its regulatory role by interacting with other regulatory proteins. Our yeast two-hybrid analysis indicated that candidate known regulatory proteins do not interact with $\mu 1 \mathrm{~A}^{1-70}$. But, by yeast two-hybrid library screening, we identified an uncharacterized protein binding with high specificity and affinity to $\mu 1 \mathrm{~A}^{1-70}$ and designated it as MuL ( $\mu 1 \mathrm{~A}$-Ligand). Preliminary in vivo analysis indicate that over-expression of $\mathrm{MuL}$ reduces the membrane recruitment of AP-1, but not that of AP-1*. A detailed functional analysis of AP-1 membrane binding in MuL 'knock-down' system and additional AP-1-MuL interaction studies should yield an understanding of its mechanism of action. 


\section{Bibliography}

Ball, C. L., Hunt, S. P., and Robinson, M. S. (1995). Expression and localization of $\alpha$-adaptin isoforms. J Cell Sci 108(8), 2865-2875. 9

Barlowe, C., Orci, L., Yeung, T., Hosobuchi, M., Hamamoto, S., Salama, N., Rexach, M. F., Ravazzola, M., Amherdt, M., and Schekman, R. (1994). COPII: a membrane coat formed by sec proteins that drive vesicle budding from the endoplasmic reticulum. Cell 77, 895-907. 2

Bonifacino, J. S. (2004). The GGA proteins: adaptors on the move. Nat Rev Mol Cell Biol 5, 23-32. 10

Bonifacino, J. S. and Glick, B. S. (2004). The mechanisms of vesicle budding and fusion. Cell 116, 153-166. 2, 15

Bonifacino, J. S. and Traub, L. M. (2003). Signals for sorting of transmembrane proteins to endosomes and lysosomes. Annu Rev Biochem 72, 395-447. 17, 56, 92

Borgne, R. L. and Hoflack, B. (1997). Mannose 6-phosphate receptors regulate the formation of clathrin-coated vesicles at the tgn. J Cell Biol 137, 335-345. 19

Chidambaram, S., Müllers, N., Wiederhold, K., Haucke, V., and von Mollard, G. F. (2004). Specific interaction between SNAREs and epsin N-terminal homology (ENTH) domains of epsin-related proteins in trans-golgi network to endosome transport. J Biol Chem 279(6), 4175-4179. 13

Claing, A., Laporte, S. A., Caron, M. G., and Lefkowitz, R. J. (2002). Endocytosis of $\mathrm{G}$ protein-coupled receptors: roles of $\mathrm{G}$ protein-coupled receptor kinases and beta-arrestin proteins. Prog Neurobiol. 9

Claussen, M., Buergisser, D., Schuller, A. G. P., Matzner, U., and Braulke, T. (1995). Regulation of insulin-like growth factor (IGF)-binding protein-6 and mannose 6phosphate/IGF-II receptor expressing in IGF-II-overexpressing NIH 3 T3 cells. Mol Endocrinol 9, 902-912. 28 
Collins, B. M., McCoy, A. J., Kent, H. M., Evans, P. R., and Owen, D. J. (2002). Molecular architecture and functional model of the endocytic AP2 complex. Cell 109(4), 523-535. 7, 73, 80

Cremona, O., Di Paolo, G., Wenk, M. R., Luthi, A., Kim, W. T., Takei, K., Daniell, L., Nemoto, Y., Shears, S. B., Flavell, R. A., McCormick, D. A., and De Camilli, P. (1999). Essential role of phosphoinositide metabolism in synaptic vesicle recycling. Cell 99(2), 179-88. 16

Crump, C. M., Xiang, Y., Thomas, L., Gu, F., Austin, C., Tooze, S. A., and Thomas, G. (2001). PACS-1 binding to adaptors is required for acidic cluster motif-mediated protein traffic. EMBO J 20, 2192-2201. 18

Dell'Angelica, E. C., Klumperman, J., Stoorvogel, W., and Bonifacino, J. S. (1998). Association of the AP-3 adaptor complex with clathrin. Science 280, 431-434. 6

Dell'Angelica, E. C., Ohno, H., Ooi, C. E., Rabinovich, E., Roche, K. W., and Bonifacino, J. S. (1997). AP-3: an adaptor-like protein complex with ubiquitous expression. EMBO J 16(5), 917-928. 5

Deneka, M., Neeft, M., Popa, I., van Oort, M., Sprong, H., Oorschot, V., Klumperman, J., Schu, P., and van der Sluijs, P. (2003). Rabaptin-5 $\alpha$ /rabaptin4 serves as a linker between rab4 and $\gamma 1$-adaptin in membrane recycling from endosomes. EMBO J 22(11), 2645-2657. 14

Dho, S. E., French, M. B., Woods, S. A., and McGlade, C. J. (1999). Characterization of four mammalian numb protein isoforms. identification of cytoplasmic and membrane-associated variants of the phosphotyrosine binding domain. $\mathrm{J}$ Biol Chem 274(46), 33097-33104. 9

Doray, B., Ghosh, P., Griffith, J., Geuze, H. J., and Kornfeld, S. (2002). Cooperation of GGAs and AP-1 in packaging MPRs at the trans-golgi network. Science 297, 1700-1703. 11, 20

Duncan, M. C., Costaguta, G., and Payne, G. S. (2003). Yeast epsin-related proteins required for Golgi-endosome traffic define a $\gamma$-adaptin ear-binding motif. Nat Cell Biol 5, 77-81. 13

Edeling, M. A., Smith, C., and Owen, D. (2006). Life of a clathrin coat: insights from clathrin and ap structures. Nat Rev Mol Cell Biol 7(1), 32-44. 4 
Erdtmann, L., Janvier, K., Rapaso, G., Craig, H. M., Benaroch, P., Berlioz Torrent, C., Guatelli, J. C., Benarous, R., and Benichou, S. (2000). Two independent regions of HIV-1 Nef are required for connection with the endocytic pathway through binding to the mu 1 chain of AP1 complex. Traffic 1, 871-883. 19

Eskelinen, E.-L., Meyer, C., Ohno, H., von Figura, K., and Schu, P. (2002). The polarized epithelia-specific $\mu 1 \mathrm{~B}$-adaptin complements $\mu 1 \mathrm{~A}$-deficiency in fibroblasts. EMBO reports 3(5), 471-477. 9

Faúndez, V., Horng, J.-T., and Kelly, R. B. (1998). A function for the AP3 coat complex in synaptic vesicle formation from endosomes. Cell 93, 423-432. 9

Fölsch, H., Ohno, H., Bonifacino, J. S., and Mellman, I. (1999). A novel clathrin adaptor complex mediates basolateral targeting in polarized epithelial cells. Cell 99, 189-198. 9

Ford, M. G., Pearse, B. M., Higgins, M. K., Vallis, Y., Owen, D. J., Gibson, A., Hopkins, C. R., Evans, P. R., and McMahon, H. T. (2001). Simultaneous binding of PtdIns(4,5)P2 and clathrin by AP180 in the nucleation of clathrin lattices on membranes. Science 291(5506), 1051-1055. 9

Ford, M. G. J., Mills, I. G., Peter, B. J., Vallis, Y., Praefcke, G. J. K., Evans, P. R., and McMahon, H. T. (2002). Curvature of clathrin-coated pits driven by epsin. Nature 419, 361-366. 13

Gaidarov, I. and Keen, J. H. (1999). Phosphoinositide-AP-2 interactions required for targeting to plasma membrane clathrin-coated pits. J Cell Biol 146, 755-764. 9

Garcia, C. K., Wilund, K., Arca, M., Zuliani, G., Fellin, R., Maioli, M., Calandra, S., Bertolini, S., Cossu, F., Grishin, N., Barnes, R., Cohen, J. C., and Hobbs, H. H. (2001). Autosomal recessive hypercholesterolemia caused by mutations in a putative LDL receptor adaptor protein. Science 292(5520), 1394-1398. 9

Ghosh, P., Griffith, J., Geuze, H. J., and Kornfeld, S. (2003). Mammalian GGAs act together to sort mannose 6-phosphate receptors. J Cell Biol 163(4), 755-766. 11

Ghosh, P. and Kornfeld, S. (2003a). AP-1 binding to sorting signals and release from clathrin-coated vesicles is regulated by phosphorylation. J Cell Biol 160(5), 699-708. 17, 80, 82, 97, 98

Ghosh, P. and Kornfeld, S. (2003b). Phosphorylation-induced conformational changes regulate GGAs 1 and 3 function at the trans-golgi network. J Biol Chem 278, 14543-14549. 10, 11, 14, 21. 
Ghosh, P. and Kornfeld, S. (2004). The cytoplasmic tail of cation-independent mannose 6-phosphate receptor contains four binding sites for AP-1. Arch Biochem Biophys 426(2). 91

Gillingham, A. K., Koumanov, F., Pryor, P. R., Reaves, B. J., and Holman, G. D. (1999). Association of ap1 adaptor complexes with GLUT4 vesicles. J Cell Sci 112(Pt 24), 4793-4800. 95

Godi, A., Di Campli, A., Konstantakopoulos, A., Di Tullio, G., Alessi, D. R., Kular, G. S., Daniele, T., Marra, P., Lucocq, J. M., and De Matteis, M. A. (2004). FAPPs control Golgi-to-cell-surface membrane traffic by binding to ARF and PtdIns(4)P. Nat Cell Biol 6(5). 3

Greene, B., Liu, S. H., Wilde, A., and Brodsky, F. M. (2000). Complete reconstitution of clathrin basket formation with recombinant protein fragments: adaptor control of clathrin self-assembly. Traffic 1(1), 69-75. 14

Griffiths, G., Back, R., and Marsh, M. (1989). A quantitative analysis of the endocytic pathway in baby hamster kidney cells. J Cell Biol 109, 2703-2720. 74

Hannan, L. A., Newmyer, S. L., and Schmid, S. L. (1998). ATP- and cytosoldependent release of adaptor proteins from clathrin-coated vesicles: a dual role for Hsc70. Mol Biol Cell 9, 2217-2229. 16

Heldwein, E. E., Macia, E., Wang, J., Yin, H. L., Kirchhausen, T., and Harrison, S. C. (2004). Crystal structure of the clathrin adaptor protein 1 core. Proc Natl Acad Sci U S A 101(39), 14108-14113. 7, 73

Hille Rehfeld, A. (1995). Mannose 6-phosphate receptors in sorting and transport of lysosomal enzymes. Biochim Biophys Acta 1241, 177-194. 19

Hinrichsen, L., Harborth, J., Andrees, L., Weber, K., and Ungewickell, E. J. (2003). Effect of clathrin heavy chain- and $\alpha$-adaptin specific small interfering RNAs on endocytic accessory proteins and receptor trafficking in HeLa cells. J Biol Chem 278(46), 45160-45170. 10

Hinrichsen, L., Meyerholz, A., Groos, S., and Ungewickell, E. J. (2006). Bending a membrane: how clathrin affects budding. Proc Natl Acad Sci U S A 103(23), 8715-8720. 5 
Hinshaw, J. E. and Schmid, S. L. (1995). Dynamin self-assembles into rings suggesting a mechanism for coated vesicle budding. Nature 374(6518), 190-192. 15

Hirst, J., Lindsay, M., and Robinson, M. S. (2001). GGAs: roles of the different domains and comparison with AP-1 and clathrin. Mol Biol Cell 12(11). 20

Hirst, J., Motley, A., Harasaki, K., Chew, S. Y. P., and Robinson, M. S. (2003). EpsinR: an ENTH domain-containing protein that interacts with AP-1. Mol Biol Cell 14, 625-641. 13

Hirst, J. and Robinson, M. S. (1998). Clathrin and adaptors. Biochim Biophys Acta 1404, 173-193. 5

Hofmann, M. W., Höning, S., Rodionov, D., Dobberstein, B., von Figura, K., and Bakke, O. (1999). The leucine-based sorting motifs in the cytoplasmic domain of the invariant chain are recognized by the clathrin adaptors AP1 and AP2 and their medium chains. J Biol Chem 274(51), 36153-36158. 18

Höning, S., Sosa, M., Hille-Rehfeld, A., and von Figura, K. (1997). The 46$\mathrm{kDa}$ mannose 6-phosphate receptor contains multiple binding sites for clathrin adaptors. J Biol Chem 272(32), 19884-19890. 19

Hooper, M., Hardy, K., Handyside, A., Hunter, S., and Monk, M. (1987). HPRTdeficient Lesch-Nyhan mouse embryos derived from germline colonization by cultured cells. Nature 326, 292-295. 26

Howell, B. W., Lanier, L. M., Frank, R., Gertler, F. B., and Cooper, J. A. (1999). The disabled 1 phosphotyrosine-binding domain binds to the internalization signals of transmembrane glycoproteins and to phospholipids. Mol Cell Biol 19(7), 51795188. 9

Huang, F., Nesterov, A., Carter, R. E., and Sorkin, A. (2001). Trafficking of yellowfluorescent-protein-tagged $\mu 1$ subunit of clathrin adaptor AP-1 complex in living cells. Traffic 2, 345-357. 20

Huttner, W. B. and Schmidt, A. (2000). Lipids, lipid modification and lipidprotein interaction in membrane budding and fission-insights from the roles of endophilin A1 and synaptophysin in synaptic vesicle endocytosis. Curr Opin Neurobiol 10(5), 543-551. 16 
Itoh, T., Koshiba, S., Kigawa, T., Kikuchi, A., Yokoyama, S., and Takenawa, T. (2001). Role of the ENTH domain in phosphatidylinositol-4,5-bisphosphate binding and endocytosis. Science 291, 1047-1051. 13

Jahn, R., Lang, T., and Südhof, T. C. (2003). Membrane fusion. Cell 112, 519-533. 17

Janvier, K., Kato, Y., Boehm, M., Rose, J. R., Martina, J. A., Kim, B.-Y., Venkatesan, S., and Bonifacino, J. S. (2003). Recognition of dileucine-based sorting signals from HIV-1 Nef and LIMP-II by the AP- $1 \gamma-\sigma 1$ and AP-3 $\delta-\sigma 3$ hemicomplexes. J Cell Biol 163(6), 1281-1290. 18, 60, 62, 93

Kent, H. M., McMahon, H. T., Evans, P. R., Benmerah, A., and Owen, D. J. (2002). $\gamma$ adaptin appendage domain: Structure and binding site for Eps15 and $\gamma$-synergin. Structure 10, 1139-1148. 13

Kirchhausen, T. (2000). Clathrin. Annu Rev Biochem 69, 699-727. 4

Kirchhausen, T. and Harrison, S. C. (1981). Protein organization in clathrin trimers. Cell 23(3), 755-761. 5

Klumperman, J., Hille, A., Veenendaal, T., Oorschot, V., Stoorvogel, W., von Figura, K., and Geuze, H. J. (1993). Differences in the endosomal distributions of the two mannose 6-phosphate receptors. J Cell Biol 121, 997-1010. 19, 28

Klumperman, J., Kuliawat, R., Griffith, J. M., Geuze, H. J., and Arvan, P. (1998). Mannose 6-phosphate receptors are sorted from immature secretory granules via adaptor protein AP-1, clathrin, and syntaxin 6-positive vesicles. J Cell Biol 141(2), 359-371. 19

Korolchuk, V. I. and Banting, G. (2002). CK2 and GAK/auxilin2 are major protein kinases in clathrin-coated vesicles. Traffic 3(6), 428-439. 82

Lefrancois, S., Janvier, K., Boehm, M., Ooi, C., and Bonifacino, J. S. (2004). An earcore interaction regulates the recruitment of the AP-3 complex to membranes. Dev Cell 7(4), 619-625. 70

Lemmon, S. K. (2001). Clathrin uncoating: Auxilin comes to life. Current Biology $11,49-52.16$

Lui, W. W. Y., Collins, B. M., Hirst, J., Motley, A., Millar, C., Schu, P., Owen, D. J., and Robinson, M. S. (2003). Binding partners for the COOH-terminal appendage domains of the GGAs and $\gamma$-adaptin. Mol Biol Cell 14, 2385-2398. 11 
Mallard, F., Antony, C., Tenza, D., Salamero, J., Goud, B., and Johannes, L. (1998). Direct pathway from early/recycling endosomes to the Golgi apparatus revealed through the study of Shiga toxin B-fragment transport. J Cell Biol 143(4), 973990. 19

Marchese, A., Chen, C., Kim, Y. M., and Benovic, J. L. (2003). The ins and outs of G protein-coupled receptor trafficking. Trends Biochem Sci 28(7), 369-376. 9

Marks, M. S., Woodruff, L., Ohno, H., and Bonifacino, J. S. (1996). Protein targeting by tyrosine- and di-leucine-based signals: Evidence for distinct saturable components. J Cell Biol 135(2), 341-354. 17, 18

Mattera, R., Arighi, C. N., Lodge, R., Zerial, M., and Bonifacino, J. S. (2003). Divalent interaction of the GGAs with the rabaptin-5-rabex-5 complex. EMBO J 22, 78-88. 11

Mattera, R., Puertollano, R., Smith, W. J., and Bonifacino, J. S. (2004). The trihelical bundle subdomain of the GGA proteins interacts with multiple partners through overlapping but distinct sites. J Biol Chem 279(30), 31409-31418. 14

Maurer, M. E. and Cooper, J. A. (2006). The adaptor protein Dab2 sorts LDL receptors into coated pits independently of AP-2 and ARH. J Cell Sci. 119(Pt 20), 4235-4246. 10

Medigeshi Ramarao, G. (2003). PhD thesis Georg-August-University Goettingen. 72, 74, 76, 96

Meyer, C., Eskelinen, E.-L., Guruprasad, M. R., von Figura, K., and Schu, P. (2001). $\mu 1 \mathrm{~A}$-deficiency induces a profound increase of MPR300/IGF-II receptor internalization rate. J Cell Sci 114, 4469-4476. 96

Meyer, C., Zizioli, D., Lausmann, S., Eskelinen, E.-L., Hamann, J., Saftig, P., von Figura, K., and Schu, P. (2000). $\mu 1 \mathrm{~A}$ adaptin-deficient mice: lethality, loss of AP-1 binding and rerouting of mannose 6-phosphate receptors. EMBO J 19, 21932203. 19, 26, 28, 52, 55, 59, 89, 90, 92, 96

Mills, I. G., Praefcke, G. J. K., Vallis, Y., Peter, B. J., Olesen, L. E., Gallop, J. L., Butler, P. J. G., Evans, P. R., and McMahon, H. T. (2003). EpsinR: an AP1/clathrin interacting protein involved in vesicle trafficking. J Cell Biol 160(2), 213-222. 13

Mishra, S. K., Keyel, P. A., Hawryluk, M. J., Agostinelli, N. R., Watkins, S. C., and Traub, L. M. (2002a). Disabled-2 exhibits the properties of a cargo-selective endocytic clathrin adaptor. Embo J . 9 
Mishra, S. K., Watkins, S. C., and Traub, L. M. (2002b). The autosomal recessive hypercholesterolemia (ARH) protein interfaces directly with the clathrin-coat machinery. Proc Natl Acad Sci U S A . 9

Molloy, S. S., Anderson, E. D., Jean, F., and Thomas, G. (1999). Bi-cycling the furin pathway: from TGN localization to pathogen activation and embryogenesis. Trends Cell Biol 9, 28-35. 19

Morris, S. M., Arden, S. D., Roberts, R. C., Kendrick-Jones, J., Cooper, J. A., Luzio, J. P., and Buss, F. (2002). Myosin VI binds to and localises with Dab2, potentially linking receptor-mediated endocytosis and the actin cytoskeleton. Traffic 3(5), 331-341. 9

Motley, A., Bright, N. A., Seaman, M. N., and Robinson, M. S. (2003). Clathrinmediated endocytosis in AP-2-depleted cells. J Cell Biol 162, 909-918. 10

Müller, W., Kuhn, R., and Rajewsky, K. (1991). Major histocompatibility complex class II hyperextension on B cells in interleukin 4-transgenic mice does not lead to B cell proliferation and hypergammaglobulinemia. Eur J Immunol 21(4), 921925. 26

Neubrand, V. E., Will, R. D., Mobius, W., Poustka, A., Wiemann, S., Schu, P., Dotti, C. G., Pepperkok, R., and Simpson, J. C. (2005). Gamma-bar, a novel AP-1interacting protein involved in post-golgi trafficking. Embo J 24(6), 1122-1133. 14,82

Nielsen, M., Madsen, P., Christensen, E. I., Nykjaer, A., Gliemann, J., Kasper, D., Pohlmann, R., and Petersen, C. (2001). The sortilin cytoplasmic tail conveys Golgi-endosome transport and binds the VHS domain of the GGA2 sorting protein. Embo J 20(9), 2180-2190. 51, 52, 65, 89, 90

Ohno, H., Aguilar, R. C., Yeh, D., Taura, D., Saito, T., and Bonifacino, J. S. (1998). The medium subunits of adaptor complexes recognize distinct but overlapping sets of tyrosine-based sorting signals. J Biol Chem 273(40), 25915-25921. 17

Ohno, H., Tomemori, T., Nakatsu, F., Okazaki, Y., Aguilar, R. C., Fölsch, H., Mellman, I., Saito, T., Shirasawa, T., and Bonifacino, J. S. (1999). $\mu 1 B$, a novel adaptor medium chain expressed in polarized epithelial cell. FEBS Lett 449, 215220. 9

Owen, D. J. and Evans, P. R. (1998). A structural explanation for the recognition of tyrosine-based endocytotic signals. Science 282, 1327-1332. 17 
Page, L. J., Sowerby, P. J., Lui, W. W. Y., and Robinson, M. S. (1999). $\gamma$-synergin: An EH domain-containing protein that interacts with $\gamma$-adaptin. J Cell Biol 146(5), 993-1004. 13

Palade, G. (1975). Intracellular aspects of the process of protein synthesis. Science 189, 347-58. 1

Pearse, B. and Robinson, M. S. (1984). Purification and properties of 100kd proteins from coated vesicles and their reconstitution with clathrin. EMBO J 4, 24572460. 5

Peden, A. A., Oorschot, V., Hesser, B. A., Austin, C. D., Scheller, R. H., and Klumperman, J. (2004). Localization of the AP-3 adaptor complex defines a novel endosomal exit site for lysosomal membrane proteins. J Cell Biol 164(7), 10651076. 6

Petersen, C., Nielsen, M., Nykjaer, A., Jacobsen, L., Tommerup, N., Rasmussen, H., Roigaard, H., Gliemann, J., Madsen, P., and Moestrup, S. K. (2001). Molecular identification of a novel candidate sorting receptor purified from human brain by receptor-associated protein affinity chromatography. J Biol Chem 272(6), 35993605. 51, 90

Prasad, K., Heuser, J., Eisenberg, E., and Greene, L. (1994). Complex formation between clathrin and uncoating ATPase. J Biol Chem 269, 6931-6939. 16

Puertollano, R., Aguilar, R. C., Gorshkova, I., Crouch, R. J., and Bonifacino, J. S. (2001). Sorting of mannose 6-phosphate receptors mediated by the GGAs. Science 292, 1712-1716. 18, 20

Puertollano, R. and Bonifacino, J. S. (2004). Interactions of GGA3 with the ubiquitin sorting machinery. Nat Cell Biol 6(3), 244-251. 11, 12

Raiborg, C., Bache, K. G., Mehlum, A., Stang, E., and Stenmark, H. (2001a). Hrs recruits clathrin to early endosomes. EMBO J 20, 5008-5021. 12

Raiborg, C., Bremnes, B., Mehlum, A., Gillooly, D. J., D’Arrigo, A., Stang, E., and Stenmark, H. (2001b). FYVE and coiled-coil domains determine the specific localisation of Hrs to early endosomes. J Cell Sci 114, 2255-2263. 12

Rapoport, I., Chen, Y. C., Cupers, P., Shoelson, S. E., and Kirchhausen, T. (1998). Dileucine-based sorting signals bind to the $\beta$ chain of AP- 1 at a site distinct and regulated differently from the tyrosine-based motif-binding site. EMBO J 17(8), 2148-2155. 18 
Reusch, U., Bernhard, O., Koszinowski, U., and Schu, P. (2002). AP-1A and AP-3A lysosomal sorting functions. Traffic 3, 752-761. 91

Reusch, U., Muranyi, W., Lucin, P., Burgert, H. G., Hengel, H., and Koszinowski, U. H. (1999). A cytomegalovirus glycoprotein re-routes MHC class I complexes to lysosomes for degradation. EMBO J 18, 1081-1091. 19

Riel, C. (2004). PhD thesis Georg-August-University Goettingen. 8, 26, 62, 68, 93, 94

Robinson, M. S. and Bonifacino, J. S. (2001). Adaptor-related proteins. Curr Opin Cell Biol 13, 444-453. 4, 5

Rodionov, D. G. and Bakke, O. (1998). Medium chains of adaptor complexes AP-1 and AP-2 recognize leucine-based sorting signals from the invariant chains. $\mathrm{J}$ Biol Chem 273, 6005-6008. 19

Rohrer, J., Schweizer, A., Russell, D., and Kornfeld, S. (1996). The targeting of lamp1 to lysosomes is dependent on the spacing of its cytoplasmic tail tyrosine sorting motif relative to the membrane. J Cell Biol 132, 565-576. 17

Royle, S. J., Bright, N. A., and Lagnado, L. (2005). Clathrin is required for the function of the mitotic spindle. Nature 434(7037), 1152-1157. 5

Sachse, M., Urbé, S., Oorschot, V., Strous, G. J., and Klumperman, J. (2002). Bilayered clathrin coats on endosomal vacuoles are involved in protein sorting toward lysosomes. Mol Biol Cell 13, 1313-1328. 12

Santolini, E., Puri, C., Salcini, A. E., Gagliani, M. C., Pelicci, P., Tacchetti, C., and Di Fiore, P. P. (2000). Numb is an endocytic protein. J cell Biol. 151(6), 13451352. 9

Schu, P. (2001). Vesicular protein transport. Pharmacogenomics J 1, 262-271. 5

Shi, J. and Kandror, K. V. (2005). Sortilin is essential and sufficient for the formation of Glut4 storage vesicles in 3T3-L1 adipocytes. Dev Cell 9(1), 99-108. 95

Shih, W., Gallusser, A., and Kirchhausen, T. (1995). A clathrin-binding site in the hinge of the beta 2 chain of mammalian AP-2 complexes. J Biol Chem 270(52), 31083-31090. 14

Shinotsuka, C., Yoshida, Y., Kawamoto, K., Takatsu, H., and Nakayama, K. (2002). Overexpression of an ADP-ribosylation factor-guanine nucleotide exchange factor, 
BIG2, uncouples Brefeldin A-induced adaptor protein-1 coat dissociation and membrane tubulation. J Biol Chem 277(11), 9468-9473. 81, 99

Simmen, T., Höning, S., Icking, A., Tikkanen, R., and Hunziker, W. (2002). AP-4 binds basolateral signals and participates in basolateral sorting in epithelial MDCK cells. Nat Cell Biol 4, 154-159. 6

Simpson, F., Peden, A. A., Christopoulou, L., and Robinson, M. S. (1997). Characterization of the adaptor-related protein complex,AP-3. J Cell Biol 137(4), 835-845. 5

Slepnev, V. and De Camilli, P. (2000). Accessory factors in clathrin-dependent synaptic vesicle endocytosis. Nat Rev Neurosci 1, 161-172. 13

Stenmark, H., Vitale, G., Ullrich, O., and Zerial, M. (1995). Rabaptin-5 is a direct effector of the small GTPase Rab5 in endocytic membrane fusion. Cell 83(3), 423-433. 14

Takatsu, H., Futatsumori, M., K.Yoshino, Yoshida, Y., Shin, H.-W., and Nakayama, K. (2001). Similar subunit interactions contribute to assembly of clathrin adaptor complexes and COPI complex: Analysis using yeast three-hybrid system. Biochem Biophys Res Commun 284, 1083-1089. 8

Takatsu, H., Sakurai, M., Shin, H. W., Murakami, K., and Nakayama, K. (1998). Identification and characterization of novel clathrin adaptor-related proteins. $\mathrm{J}$ Biol Chem 273(38), 24693-24700. 8

Tooze, S. A., Martens, G. J., and Huttner, W. B. (2001). Secretory granule biogenesis: rafting to the SNARE. Trends Cell Biol 11(3). 3

Umeda, A., Meyerholz, A., and Ungewickell, E. (2000). Identification of the universal cofactor (auxilin 2) in clathrin coat dissociation. Eur J Cell Biol 79, 336-342. 82

Ungewickell, E., Ungewickell, H., Holstein, S. E., Lindner, R., Prasad, K., Barouch, W., Martin, B., Greene, L. E., and Eisenberg, E. (1995). Role of auxilin in uncoating clathrin-coated vesicles. Nature 378, 632-635. 16

Waguri, S., Dewitte, F., Borgne, R. L., Rouillé, Y., Uchiyama, Y., Dubremetz, J.-F., and Hoflack, B. (2003). Visualization of TGN to endosome trafficking through fluorescently labeled MPR and AP-1 in living cells. Mol Biol Cell 14, 142-155. 20 
Wan, L., Molloy, S. S., Thomas, L., Liu, G., Xiang, Y., Rybak, S. L., and Thomas, G. (1998). PACS-1 defines a novel gene family of cytosolic sorting proteins required for trans Golgi network localization. Cell 94, 205-216. 18

Wendland, B. (2002). Epsins: adaptors in endocytosis? Nat Rev Mol Cell Biol 3(12), 971-977. 9

Wilde, A. and Brodsky, F. M. (1996). In vivo phosphorylation of adaptors regulates their interaction with clathrin. J Cell Biol 135. 14

Wu, X., Zhao, X., Baylor, L., Kaushal, S., Eisenberg, E., and Greene, L. E. (2001). Clathrin exchange during clathrin-mediated endocytosis. J Cell Biol 155(2), 291300. 76, 97

Wu, X., Zhao, X., Puertollano, R., Bonifacino, J. S., Eisenberg, E., and Greene, L. E. (2003). Adaptor and clathrin exchange at the plasma membrane and trans-Golgi network. Mol Biol Cell 14(2), 516-528. 5, 76, 77, 97

Yasukawa, T., Kanei-Ishii, C., Maekawa, T., Fujimoto, J., Yamamoto, T., and Ishii, S. (1995). Increase of solubility of foreign proteins in escherichia coli by coproduction of bacterial thioredoxin. J Biol Chem 270(43), 25328-25331. 25

Zhang, B., Koh, Y. H., Beckstead, R. B., Budnik, V., Ganetzky, B., and Bellen, H. J. (1998). Synaptic vesicle size and number are regulated by a clathrin adaptor protein required for endocytosis. Neuron 21(6), 1465-1475. 10

Zhu, Y., Doray, B., Poussu, A., Lehto, V. P., and Kornfeld, S. (2001). Binding of GGA2 to the lysosomal enzyme sorting motif of the mannose 6-phosphate receptor. Science 292, 1716-1718. 18 


\section{Acknowledgements}

At the outset, I would like to thank Prof. Dr. Kurt von Figura, for giving me an opportunity to work in this institute and for being a source of inspiration. I thank Prof. Dr. Fritz, for agreeing to be my co-referee.

I thank Prof. Dr. Peter Schu for his support, guidance and for being a good mentor. During these three years, I had learnt the art of critical scientific thinking and well thought experimental planning. Thanks again for your patience and guidance in the completion of this thesis.

With great pleasure, I would like to thank my colleagues who extended their help with all their kindness and patience and for creating a lively working atmosphere in the lab. Thank you Olaf and Jutta for all your help with the experiments. Olaf, thanks for all the good discussions we had during our coffee breaks. I thank Dr. Constanze Riel, for her help during the early stages of my work. Thank you Rekha for being a good friend.

I would like to thank both Tanja Benkert and Jennifer Baltes for being so kind to be always there during hardships not only at work, but outside the lab too. Thank you Jenny and Tanja for being critical towards my mistakes, which I appreciate, for it has improved my working style.

I thank Prof. Dr. Torben Luebcke and his group members Katrin, Ellen, Florian and Markus for their help and discussions. I thank Prof. Dr. Michael Thumm and his group for their help with my yeast experiments. I would like to thank Prof. Dr. Thomas Dierks and Dr. Bernhard Schmidt for their help.

Special thanks to Dr. Malaiyalam, and Santhosh lakshmi for all the scientific discussions we had, which for sure helped me to work better. Thank you Mals and santosh for your all your help. Thank you Prem for being such a caring friend. I would like to thank Sridhar Sriramulu and Mals for their timely help with their 
expertise in LaTex, which helped me to successfully finish writing the thesis.

I would like to thank my friends Krishna, Ajay, Sunil, Rajesh and Chakravarthi for all their help and being nice friends.

This work would not be possible without the moral support and care of my parents and my sister. I extend my gratitude to them and other relatives. 


\section{Curriculum Vitae}

Name: Radhakrishnan Karthikeyan.

Date of Birth: 16.11 .1976$.

Father's Name: Mr. Radhakrishnan Viswanathan.

Mother's Name: Mrs. Saraswathi Radhakrishnan .

Place of Birth: Krishnagiri, India.

1992 - 1994: High School, Krishnagiri.

1994 - 1997: Bachelor of Science in Biochemistry, Islamiah college, Vaniyambadi, India

1997 - 1999: Master of Science in Biochemistry, University of Madras, India.

July 1999 - Aug 2000: Worked as Junior Research Fellow with Prof. P.V.

Sundaram, PROMED, Chennai, India.

Sep 2000 - Aug 2002: Worked as Junior Research Fellow in Institute of

Chemistry, Academia Sinica, Taipei, Taiwan .

Apr 2003 - Present Doctorate student under Prof. Dr. Peter Schu, Biochemistry

II, University of Goettingen. 